\title{
LARGE DEVIATIONS FOR DIFFUSIONS INTERACTING THROUGH THEIR RANKS
}

\author{
AMIR DEMBO, MYKHAYLO SHKOLNIKOV, S. R. SRINIVASA VARADHAN, \\ AND OFER ZEITOUNI
}

\begin{abstract}
We prove a Large Deviations Principle (LDP) for systems of diffusions (particles) interacting through their ranks, when the number of particles tends to infinity. We show that the limiting particle density is given by the unique solution of the appropriate McKean-Vlasov equation and that the corresponding cumulative distribution function evolves according to a non-degenerate generalized porous medium equation with convection. The large deviations rate function is provided in explicit form. This is the first instance of a LDP for interacting diffusions, where the interaction occurs both through the drift and the diffusion coefficients and where the rate function can be given explicitly. In the course of the proof, we obtain new regularity results for tilted versions of such generalized porous medium equation.
\end{abstract}

\section{INTRODUCTION}

Recently, systems of diffusion processes (particles) interacting through their ranks have received much attention. For a fixed number of particles $N \in \mathbb{N}$, these are given by the unique weak solution of the stochastic differential system (SDS),

$$
\mathrm{d} X_{i}(t)=\sum_{j=1}^{N} b_{j} \mathbf{1}_{\left\{X_{i}(t)=X_{(j)}(t)\right\}} \mathrm{d} t+\sum_{j=1}^{N} \sigma_{j} \mathbf{1}_{\left\{X_{i}(t)=X_{(j)}(t)\right\}} \mathrm{d} W_{i}(t), \quad i=1, \ldots, N,
$$

where $b_{1}, b_{2}, \ldots, b_{N}$ are arbitrary real constants, $\sigma_{1}, \sigma_{2}, \ldots, \sigma_{N}$ are arbitrary positive constants, $W_{1}, W_{2}, \ldots, W_{N}$ are independent standard Brownian motions and $X_{(1)}(t) \leq$ $X_{(2)}(t) \leq \ldots \leq X_{(N)}(t)$ are the ordered particles at time $t$. In this paper we study the behavior of the solution to (1.1) as $N$ becomes large in the regime when $\left|b_{j+1}-b_{j}\right|+$ $\left|\sigma_{j+1}^{2}-\sigma_{j}^{2}\right|=O\left(N^{-1}\right)$ for all $j$ (see Assumption 1.2 below for the details). In other words, the drift and the diffusion coefficients of a particle change slowly as it changes its rank in the particle system.

The existence and uniqueness of the weak solution to (1.1) was shown in the work [3], which was motivated by questions in filtering theory. The system (1.1) has also reappeared in the context of stochastic portfolio theory under the name first-order market model (see the book [14] and the survey article [15]). In the latter context, our choice of the regime $\left|b_{j+1}-b_{j}\right|+\left|\sigma_{j+1}^{2}-\sigma_{j}^{2}\right|=O\left(N^{-1}\right)$ agrees with the economic intuition that a small change of a company's rank cannot lead to a large jump in the growth rate and the volatility coefficient of its market capitalization. Due to its central role in the analysis of capital distributions in financial markets and long-term portfolio performance therein, as well as its intriguing mathematical features, the ergodicity and

We thank N. Krylov for suggesting the method applied in the proof of Lemma 3.3 (which is a key ingredient in the proof of Prop 2.2(A)) and for describing to one of us some of the details needed for the implementation of the method. Our research was partially supported by NSF grants \#DMS1106627 (Dembo and Shkolnikov), \#DMS-1208334 (Varadhan), \#DMS-0804133 (Zeitouni), and the Israel Science Foundation grant \#111/11 (Zeitouni). 
sample path properties of this model have undergone a detailed analysis in the case that the number of particles is fixed (see [6, 7, 19, 20, 21]). Moreover, concentration properties of the solution to (1.1) for large values of $N$ have been studied in [34, and an analogous infinite particle system has been constructed and analyzed in 33 .

In [36] it was observed that the SDS (1.1) can be rewritten as

$$
\mathrm{d} X_{i}(t)=b\left(F_{\rho^{N}(t)}\left(X_{i}(t)\right)\right) \mathrm{d} t+\sigma\left(F_{\rho^{N}(t)}\left(X_{i}(t)\right)\right) \mathrm{d} W_{i}(t), \quad i=1,2, \ldots, N,
$$

where $\rho^{N}(t)=\frac{1}{N} \sum_{i=1}^{N} \delta_{X_{i}(t)}$ is the empirical measure of the particle system at time $t$, $F_{\rho^{N}(t)}$ is the corresponding cumulative distribution function $(\mathrm{CDF})$, and $b:[0,1] \rightarrow \mathbb{R}$, $\sigma:[0,1] \rightarrow(0, \infty)$ are functions satisfying $b\left(\frac{j}{N}\right)=b_{j}, \sigma\left(\frac{j}{N}\right)=\sigma_{j}$ for all $j=$ $1,2, \ldots, N$. The representation (1.2) allows us to view the particle system (1.1) as a system of diffusion processes interacting through their mean-field. It gives rise to questions on the large $N$ behavior of the empirical measure in (1.2), where the mathematical challenge is due to the discontinuity of the diffusion coefficients in (1.2). A Law of Large Numbers (LLN) for $\rho^{N}(t)$ is obtained in [36] for non-decreasing $i \mapsto X_{i}(0)$, with $\left\{X_{i}(0)-X_{1}(0)\right\}$ chosen according to the stationary distribution of the process of spacings between consecutive ordered particles in (1.2) (in particular, assuming that $b$ and $\sigma$ are such that a stationary distribution exists). In this case, it was shown that the limiting particle measure path $t \mapsto \gamma(t)(\cdot)$ satisfies the McKean-Vlasov equation

$$
\int_{\mathbb{R}} f \mathrm{~d} \gamma(t)-\int_{\mathbb{R}} f \mathrm{~d} \gamma(0)=\int_{0}^{t} \mathrm{~d} s \int_{\mathbb{R}}\left[b\left(F_{\gamma(s)}(\cdot)\right) f^{\prime}+\frac{1}{2} \sigma\left(F_{\gamma(s)}(\cdot)\right)^{2} f^{\prime \prime}\right] \mathrm{d} \gamma(s)
$$

for all Schwartz functions $x \mapsto f(x)$ and any $t \geq 0$ (hereafter $\mathrm{d} \gamma(s)$ is short hand for integration with respect to the probability measure $\gamma(s)(\cdot)$ on $\mathbb{R}$, at the fixed time $s$ ). Further, the corresponding CDF-path $R^{(\gamma)}(t, x):=F_{\gamma(t)}(x)$ evolves according to the non-degenerate generalized porous medium equation with convection (see [37] and the references therein for a thorough treatment),

$$
R_{t}=(\Sigma(R))_{x x}-(\Gamma(R))_{x}
$$

where $\Sigma(r)=\int_{0}^{r} \frac{1}{2} \sigma^{2}(u) \mathrm{d} u$ and $\Gamma(r)=\int_{0}^{r} b(u) \mathrm{d} u$.

In this paper, we establish a Large Deviations Principle (LDP) for the sequence $\left\{\rho^{N}\right.$, $N \in \mathbb{N}\}$ of paths $\left\{\rho^{N}(t), t \in[0, T]\right\}$ of empirical measures, where $T>0$ is arbitrary, but fixed throughout. Among other things, this LDP implies the LLN for $\left\{\rho^{N}, N \in \mathbb{N}\right\}$. Such LLN, and the LDP upper bound are shown under the mild regularity Asmp. 1.2 on the functions $b, \sigma$ and the initial empirical measures $\left\{\rho^{N}(0), N \in \mathbb{N}\right\}$ (dispensing of the stationarity assumption on the process of spacing, which plays a crucial role in [36]). Our next definition is useful for stating Asmp. 1.2

Definition 1.1. Let $M_{1}(\mathbb{R})$ denote the space of Borel probability measures on $\mathbb{R}$, endowed with the Lévy distance metric

$$
d_{L}\left(\alpha_{1}, \alpha_{2}\right):=\inf \left\{\epsilon>0 \mid \alpha_{1}(O) \leq \alpha_{2}\left(O_{\epsilon}\right)+\epsilon, \alpha_{2}(O) \leq \alpha_{1}\left(O_{\epsilon}\right)+\epsilon \forall \text { open } O \subset \mathbb{R}\right\}
$$

(where $O_{\epsilon}$ stands for the $\epsilon$-neighborhood of $O$ in $\mathbb{R}$ ). Then, for $\iota \in[0,1]$ let $M_{1}^{(\iota)}(\mathbb{R})$ denote the subset of all $\mu \in M_{1}(\mathbb{R})$ such that $\int_{\mathbb{R}}|x|^{1+\iota} \mathrm{d} \mu<\infty$ and $\frac{\mathrm{d} \mu}{\mathrm{d} x} \in L^{q_{0}}(\mathbb{R})$ for some $q_{0}>1$.

Assumption 1.2. $\sigma_{j}=\sigma\left(\frac{j}{N}\right)$ and $b_{j}=b\left(\frac{j}{N}\right)$, where:

(a) The function $A:=\frac{1}{2} \sigma^{2}$ is uniformly bounded below by some $\underline{a}>0$, and $A^{\prime}$ is bounded on $[0,1]$.

(b) The function $b(\cdot)$ is Lipschitz continuous on $[0,1]$. 
(c) As $N \rightarrow \infty$ the deterministic initial empirical measures $\left\{\rho^{N}(0)\right\}$ converge weakly to some $\rho_{0} \in M_{1}^{\left(\iota_{\star}\right)}(\mathbb{R}), \iota_{\star} \in(0,1]$, and $\sup _{N \in \mathbb{N}} \int_{\mathbb{R}}|x|^{1+\iota_{\star}} \mathrm{d} \rho^{N}(0)<\infty$.

(d) The function $A^{\prime}$ is further Lipschitz continuous on $[0,1]$.

Throughout the paper we let $\mathcal{C}=C\left([0, T], M_{1}(\mathbb{R})\right)$ stand for the space of continuous functions from $[0, T]$ to $M_{1}(\mathbb{R})$ endowed with the metric

$$
d\left(\gamma_{1}(\cdot), \gamma_{2}(\cdot)\right)=\sup _{t \in[0, T]} d_{L}\left(\gamma_{1}(t), \gamma_{2}(t)\right)
$$

Further, throughout we adopt the convention that $\frac{0}{0}=0$, and identify each $\gamma \in \mathcal{C}$ with the corresponding CDF-path $R^{(\gamma)}(t, x):=F_{\gamma(t)}(x)$. The following spaces of functions then play a major role in our LDP on $\mathcal{C}$.

Definition 1.3. Let $\overline{\mathcal{S}}$ denote the space of functions $g$ on $\mathbb{R}_{T}:=[0, T] \times \mathbb{R}$ which are infinitely differentiable and such that, for all $t \in[0, T], g(t, \cdot)$ is a Schwartz function on $\mathbb{R}$. Next, let

$$
\begin{aligned}
& \widetilde{\mathcal{A}}:=\left\{\gamma \in \mathcal{C}: R^{(\gamma)} \in C_{b}\left(\mathbb{R}_{T}\right), \quad \gamma(0) \in M_{1}^{(0)}(\mathbb{R}),\right. \\
& t\left.\mapsto \int_{\mathbb{R}} g(t, \cdot) \mathrm{d} \gamma(t) \quad \text { abs. cont. on }[0, T] \forall g \in \overline{\mathcal{S}}\right\},
\end{aligned}
$$

and for each $\iota \in(0,1], \mu \in M_{1}^{(0)}(\mathbb{R})$, its subset

$$
\mathcal{A}_{\iota, \mu}:=\left\{\gamma \in \widetilde{\mathcal{A}}: \gamma(0)=\mu, \int_{\mathbb{R}_{T}}|x|^{1+\iota} \mathrm{d} \gamma(t) \mathrm{d} t<\infty\right\} .
$$

Further, we use

$$
\mathcal{F}_{q}:=\left\{R=R^{(\gamma)}: \gamma \in \mathcal{C},\right.
$$

with $\mathcal{F}:=\mathcal{F}_{3 / 2}$ and

$$
\left.R_{t}, R_{x x} \in L^{q}\left(\mathbb{R}_{T}\right), R_{x} \in L^{3}\left(\mathbb{R}_{T}\right), \frac{R_{t}^{2}}{R_{x}}, \frac{R_{x x}^{2}}{R_{x}} \in L^{1}\left(\mathbb{R}_{T}\right)\right\}
$$

$$
\widetilde{J}(\gamma):= \begin{cases}\frac{1}{4}\left\|\frac{R_{t}-\left(A(R) R_{x}\right)_{x}+b(R) R_{x}}{\left(A(R) R_{x}\right)^{1 / 2}}\right\|_{L^{2}\left(\mathbb{R}_{T}\right)}^{2}, & \text { if } \gamma \in \widetilde{\mathcal{A}}, R=R^{(\gamma)} \in \mathcal{F} \\ \infty, & \text { otherwise },\end{cases}
$$

with $J_{\iota, \mu}(\gamma)$ defined as in (1.8) except for replacing there $\widetilde{\mathcal{A}}$ by the smaller $\mathcal{A}_{\iota, \mu}$.

Our main result then reads as follows.

Theorem 1.4. Under Asmp. 1.2 with $\iota_{\star}=1$, the sequence $\left\{\rho^{N}, N \in \mathbb{N}\right\}$ satisfies the LDP on $\mathcal{C}$ with scale $N$ and good rate function $J_{1, \rho_{0}}(\cdot)$ of Definition 1.3.

Remark 1.5. As shown in Prop. 2.6 (and Corollary 2.3), the exponential tightness of $\left\{\rho^{N}\right\}$ and the LDP upper bound of Theorem 1.4 with rate $J_{\iota_{\star}, \rho_{0}}(\cdot)$ apply for any value of $\iota_{\star}>0$ in Asmp. 1.2(c) and do not require part (d) of Asmp. 1.2.

In view of the preceding remark we have the following LLN.

Corollary 1.6. Under Asmp. 1.2(a)-(c) for some $\iota_{\star} \in(0,1]$, the sequence $\left\{\rho^{N}\right.$, $N \in \mathbb{N}\}$ converges almost surely to the unique path $\gamma_{\star} \in \mathcal{A}_{\iota_{\star}, \rho_{0}}$ for which $R^{\left(\gamma_{\star}\right)} \in \mathcal{F}$ is a generalized solution of the Cauchy problem

$$
R_{t}=\left(A(R) R_{x}\right)_{x}-b(R) R_{x}, \quad R(0, \cdot)=F_{\rho_{0}}(\cdot) .
$$


Proof. Setting $J:=J_{\iota_{\star}, \rho_{0}}$, recall Remark 1.5 that the exponentially tight $\left\{\rho^{N}\right\}$ satisfy the LDP upper bound in the metric space $(\mathcal{C}, d)$ with some rate function $I_{\iota_{\star}, \rho_{0}}(\cdot) \geq J(\cdot)$. Necessarily, $I_{\iota_{\star}, \rho_{0}}$ has compact, non-empty, level sets and in particular $J^{-1}(0)=\{\gamma \in$ $\mathcal{C}: J(\gamma)=0\}$ is non-empty (and pre-compact). Considering the LDP upper bound for the complement of any finite $\delta$-cover of $J^{-1}(0)$, we further deduce by the first BorelCantelli lemma that a.s. $d\left(\rho^{N}, J^{-1}(0)\right) \rightarrow 0$. From the explicit formula (1.8) we know that $J^{-1}(0) \subseteq \mathcal{A}_{\iota_{\star}, \rho_{0}}$ and further to each $\gamma \in J^{-1}(0)$ corresponds $R^{(\gamma)} \in \mathcal{F}$ which is a non-negative continuous bounded generalized solution of the problem (1.9), in the sense of [18, Definition 4]. Recall [18, Theorem 4] that such generalized solution is unique. Consequently, $J^{-1}(0)=\left\{\gamma_{\star}\right\}$ is a single point to which $\rho^{N}$ converges a.s. when $N \rightarrow \infty$.

In [8], the authors prove a LDP for systems of diffusions with the same constant diffusion coefficient, where further each drift coefficient is a continuous function of the value of the diffusion and the empirical measure of the whole system. In this context the (local) LDP is established by a clever application of Girsanov's theorem, which allows one to move from the system of interacting diffusions to the corresponding system of independent diffusions (on the event that the path of empirical measures is near a deterministic path of probability measures). Such an approach is not viable in our case because of the interaction through the diffusion coefficients in (1.2). Moreover, the discontinuity of the drift and the diffusion coefficients presents an additional challenge. Even on the level of the LLN as in Corollary 1.6. previous works had to assume that there is no interaction through the diffusions coefficients (see [4, 5, 22 . and the references therein), or be restricted to special initial conditions (see [36]). We overcome these challenges, but remark that our analysis relies on the particular form of the drift and the diffusion coefficients in (1.2).

A crucial part of the proof of Theorem 1.4 is devoted to the study of generalized solutions to porous medium equations with tilt:

$$
R_{t}=\left(A(R) R_{x}\right)_{x}+h A(R) R_{x} .
$$

The following regularity result, which we need in the proof of Theorem 1.4, is also of independent interest.

Theorem 1.7. Let $R \in C_{b}\left(\mathbb{R}_{T}\right)$ be such that, for every $t \in[0, T]$, the function $R(t, \cdot)$ is a CDF of a probability measure $\gamma(t)$. Suppose that $R$ is a generalized solution to (1.10) with initial condition $R(0, \cdot)=F_{\mu}(\cdot)$, where $A(\cdot)$ satisfies Asmp. 1.2 $(a), \mu \in M_{1}^{(0)}(\mathbb{R})$ and $h$ is a function on $\mathbb{R}_{T}$ such that

$$
\int_{\mathbb{R}_{T}} h^{2}(t, x) \mathrm{d} \gamma(t) \mathrm{d} t<\infty .
$$

If, in addition, $\gamma(\cdot)$ satisfies the moment condition (1.6) for some $\iota>0$, then $\gamma \in \mathcal{A}_{\iota, \mu}$ and $R \in \mathcal{F}_{q}$ of (1.7), for all $\frac{6}{5} \leq q \leq \frac{3}{2}$.

\section{Outline: Proofs of Theorems 1.4 and 1.7}

In this section we establish Theorems 1.4 and 1.7 as consequences of Propositions 2.2 and 2.5 2.7. The latter are in turn proved in the following five sections, in the order in which they are stated here. We note in passing that the proofs of Propositions 2.2 and 2.5 are of analytic nature (relying for proving Prop. 2.2 on results from [26, 27, 29] about parabolic equations with non-smooth coefficients), whereas those of Propositions 2.6 and 2.7 are mostly probabilistic, involving tools from large deviations 
theory and stochastic analysis. More precisely, the local large deviations upper bound of Prop. 2.6 is established by integrating suitable test functions against $\rho^{N}$, proving a Freidlin-Wentzell type local large deviations upper bound for the resulting processes and optimizing over such test functions; and the local large deviations lower bound of Prop. 2.7 is shown via a tilting argument which relies on an appropriate Girsanov change of measure.

We proceed with few notations and definitions that are used throughout this paper. First, we write $m$ for the Lebesgue measure on $\mathbb{R}_{T}=[0, T] \times \mathbb{R},(\alpha, f)$ for $\int_{\mathbb{R}} f \mathrm{~d} \alpha$, any $f$ in the space of continuous bounded functions $C_{b}(\mathbb{R})$ and any $\alpha \in M_{1}(\mathbb{R})$, with $(\alpha, f)(s)=\int_{\mathbb{R}} f(s, \cdot) \mathrm{d} \alpha(s, \cdot)$, in case of $s \mapsto f(s, \cdot) \in C_{b}(\mathbb{R})$ and $s \mapsto \alpha(s, \cdot) \in M_{1}(\mathbb{R})$ (or more generally, whenever $f(s, \cdot)$ is integrable with respect to $\alpha(s, \cdot)$ ). We further let $\overline{\mathcal{S}}_{x}=\left\{g_{x}: g \in \overline{\mathcal{S}}\right\}$ denote the space of spatial derivatives of test functions from $\overline{\mathcal{S}}$.

Definition 2.1. Setting

$$
\mathcal{R}^{\gamma} g=g_{t}+b\left(R^{(\gamma)}\right) g_{x}+A\left(R^{(\gamma)}\right) g_{x x},
$$

with the functional

$$
\Phi_{\gamma}(t, g)=(\gamma, g)(t)-(\gamma, g)(0)-\int_{0}^{t}\left(\gamma, \mathcal{R}^{\gamma} g\right)(s) \mathrm{d} s
$$

on $\overline{\mathcal{S}}, \Phi_{\gamma}(g):=\Phi_{\gamma}(T, g)$, and inner product

$$
(f, g)_{\gamma}=\int_{\mathbb{R}_{T}} f_{x} g_{x} A\left(R^{(\gamma)}\right) \mathrm{d} \gamma(t) \mathrm{d} t
$$

on $\overline{\mathcal{S}}$, we consider the (rate) functions on $\mathcal{C}$ given by

$$
I_{\iota, \mu}(\gamma)= \begin{cases}\sup _{g \in \overline{\mathcal{S}}}\left[\Phi_{\gamma}(g)-(g, g)_{\gamma}\right], & \gamma \in \mathcal{A}_{\iota, \mu} \\ \infty, & \text { otherwise }\end{cases}
$$

Theorem 1.7 is a direct consequence of the following proposition (which is also key to proving Theorem 1.4).

Proposition 2.2. Suppose Asmp. 1.2(a) holds and the function $b(\cdot)$ is uniformly bounded. If $I_{\iota, \mu}(\gamma)<\infty$ for some $\iota>0$ and $\mu \in M_{1}^{(0)}(\mathbb{R})$, then $R^{(\gamma)} \in \mathcal{F}_{q}$ for all $\frac{6}{5} \leq q \leq \frac{3}{2}$. Namely, $R^{(\gamma)}=R$ such that:
(A) $R_{x} \in L^{3}\left(\mathbb{R}_{T}\right)$,
(B) $R_{t}, R_{x x} \in L^{q}\left(\mathbb{R}_{T}\right)$ for all $\frac{6}{5} \leq q \leq \frac{3}{2}$,
(C) $\int_{\mathbb{R}_{T}} \frac{R_{x x}^{2}}{R_{x}} \mathrm{~d} m<\infty, \int_{\mathbb{R}_{T}} \frac{R_{t}^{2}}{R_{x}} \mathrm{~d} m<\infty$.

Proof of Theorem 1.7. After integration by parts in space, we see that having $R=R^{(\gamma)}$ as generalized solution of (1.10), is equivalent to

$$
\Phi_{\gamma}(t, g)=-\int_{0}^{t}\left(\gamma, b(R) g_{x}+h A(R) g_{x}\right)(s) \mathrm{d} s,
$$

for any $g \in \overline{\mathcal{S}}$ and $t \in[0, T]$. In particular, upon comparing (2.2) and (2.5), we deduce that $t \mapsto(\gamma, g)(t)$ is absolutely continuous for any $g \in \overline{\mathcal{S}}$, and thus $\gamma \in \mathcal{A}_{\iota, \mu}$ (for $R \in C_{b}\left(\mathbb{R}_{T}\right)$, and the moment and initial conditions have all been assumed in Theorem [1.7). Further, taking here $b \equiv 0$ without loss of generality, we see that for $\gamma$ 
as in Theorem 1.7,

$$
\begin{aligned}
I_{\iota, \mu}(\gamma) & =\sup _{f \in \overline{\mathcal{S}}_{x}}\left[\int_{\mathbb{R}_{T}}\left(-h A(R) f-A(R) f^{2}\right) \mathrm{d} \gamma(t) \mathrm{d} t\right] \\
& \leq \sup _{f \in L^{2}\left(\mathbb{R}_{T}, \mathrm{~d} \gamma(t) \mathrm{d} t\right)}\left[\int_{\mathbb{R}_{T}}\left(-h A(R) f-A(R) f^{2}\right) \mathrm{d} \gamma(t) \mathrm{d} t\right] .
\end{aligned}
$$

The latter supremum is attained for $f=-\frac{1}{2} h$ and its value is finite due to our assumption (1.11). Consequently, in this case $I_{\iota, \mu}(\gamma)<\infty$ and by Prop. 2.2 such $R=R^{(\gamma)}$ satisfies the regularity properties (1.7) for all $\frac{6}{5} \leq q \leq \frac{3}{2}$, as claimed.

We start the proof of Theorem 1.4 by establishing the following corollary of Prop. 2.2 .

Corollary 2.3. Suppose $A(\cdot)$ satisfies Asmp. 1.2 $(a), \mu \in M_{1}^{(0)}(\mathbb{R})$ and $b(\cdot)$ is uniformly bounded. If $I_{\iota, \mu}(\gamma)<\infty$ then $J_{\iota, \mu}(\gamma)=I_{\iota, \mu}(\gamma)$. In particular, $J_{\iota, \mu}(\gamma) \leq I_{\iota, \mu}(\gamma)$ for all $\gamma \in \mathcal{C}$.

Proof. Fixing $\gamma \in \mathcal{A}_{\iota, \mu}$ with

$$
I_{\iota, \mu}(\gamma)=\sup _{g \in \mathcal{S}}\left[\Phi_{\gamma}(g)-(g, g)_{\gamma}\right]<\infty,
$$

consider the Hilbert space $\mathbb{H}$ given by identifying and completing $\overline{\mathcal{S}}$ under the seminorm corresponding to the inner product $(\cdot, \cdot)_{\gamma}$ of $(2.3)$. By scaling, the linear functional $\Phi_{\gamma}(\cdot)$ is bounded on $\overline{\mathcal{S}}$ by $2 \sqrt{I_{\iota, \mu}(\gamma)}$ times this semi-norm, and with $A(\cdot)$ uniformly bounded below, if $(g, g)_{\gamma}=0$ then by (2.1)-(2.3) also $\Phi_{\gamma}(g)=0$. Hence, there exists a unique bounded linear functional $\bar{\Phi}_{\gamma}$ on $\mathbb{H}$ which coincides with $\Phi_{\gamma}$ on $\overline{\mathcal{S}}$. Now, by the Riesz representation theorem, there is a unique element $\tilde{h} \in \mathbb{H}$, which satisfies $\bar{\Phi}_{\gamma}(g)=(\tilde{h}, g)_{\gamma}$ for all $g \in \mathbb{H}$. Combining this with the fact that $\overline{\mathcal{S}}$ is by definition dense in $\mathbb{H}$, we obtain that

$$
I_{\iota, \mu}(\gamma)=\sup _{g \in \mathbb{H}}\left[\Phi_{\gamma}(g)-(g, g)_{\gamma}\right]=\sup _{g \in \mathbb{H}}\left[(\tilde{h}, g)_{\gamma}-(g, g)_{\gamma}\right]=\frac{1}{4}(\tilde{h}, \tilde{h})_{\gamma} .
$$

Furthermore, by the definition of $\tilde{h}$ and $\Phi_{\gamma}$, we have that $\tilde{h}_{x} \in L^{2}\left(\mathbb{R}_{T}, \mathrm{~d} \gamma(t) \mathrm{d} t\right)$ satisfies

$$
\Phi_{\gamma}(t, g)=\int_{0}^{t}\left(\gamma, A\left(R^{(\gamma)}\right) \tilde{h}_{x} g_{x}\right)(s) \mathrm{d} s
$$

for $t=T$ and any $g \in \overline{\mathcal{S}}$. In particular, considering Schwartz functions $g$ supported on $\mathbb{R}_{t}$ we have that (2.7) applies also for any $t \in[0, T]$. Comparing this with (2.5) we deduce that $R=R^{(\gamma)}$ is a generalized solution of the PDE (1.10) for

$$
h=-\tilde{h}_{x}-\frac{b(R)}{A(R)} .
$$

By the assumed boundedness of $\frac{b}{A}$, clearly $h \in L^{2}\left(\mathbb{R}_{T}, \mathrm{~d} \gamma(t) \mathrm{d} t\right)$. By Theorem 1.7, this implies in turn that $R_{t}, R_{x x}$ and the $L^{1}\left(\mathbb{R}_{T}\right)$ density $R_{x}$ are elements of $L^{3 / 2}\left(\mathbb{R}_{T}\right)$ and, moreover, the functions $R_{t} R_{x}^{-1 / 2}, R_{x}^{3 / 2}$ and $R_{x x} R_{x}^{-1 / 2}$ are elements of $L^{2}\left(\mathbb{R}_{T}\right)$. Thus, the identity

$$
h\left(A(R) R_{x}\right)^{1 / 2}=\frac{R_{t}-\left(A(R) R_{x}\right)_{x}}{\left(A(R) R_{x}\right)^{1 / 2}}
$$


holds in $L^{2}\left(\mathbb{R}_{T}\right)$. Finally, putting (2.9),$(2.8)$ and (2.6) together, we end up with

$$
I_{\iota, \mu}(\gamma)=\frac{1}{4}\left\|\widetilde{h}_{x}\left(A\left(R^{(\gamma)}\right) R_{x}^{(\gamma)}\right)^{1 / 2}\right\|_{L^{2}\left(\mathbb{R}_{T}\right)}=J_{\iota, \mu}(\gamma)
$$

of (1.8), as claimed.

Corollary 2.3 allows us to replace the function $I_{1, \rho_{0}}(\cdot)$ of the large deviations upper bound for Theorem 1.4 (see (2.13) ), by $J_{1, \rho_{0}}(\cdot)$ of the corresponding lower bound (see Prop. 2.7). The task of proving such lower bound is further simplified thanks to the next proposition, for which we first introduce some relevant notations.

Definition 2.4. Let $\mathcal{G}$ denote the subset of $\{\gamma \in \mathcal{C}: \widetilde{J}(\gamma)<\infty\}$ for which $R:=R^{(\gamma)} \in$ $C_{b}^{\infty}\left(\mathbb{R}_{T}\right), R_{x}$ is strictly positive and (1.10) holds point-wise for some $h \in C_{b}\left(\mathbb{R}_{T}\right)$ with $x \mapsto h(t, x)$ uniformly Lipschitz continuous on $\mathbb{R}_{T}$.

Proposition 2.5. Suppose Asmp. 1.2(a),(b) and (d) hold, and that $J_{1, \mu}(\gamma)<\infty$ for some $\mu \in M_{1}^{(1)}(\mathbb{R})$. Then, there exist $\gamma^{\ell, \epsilon}=\left(1-\ell^{-1}\right) \gamma^{\epsilon}+\ell^{-1} \widehat{\mu}^{\epsilon} \in \mathcal{G}$ for some $\widehat{\mu}^{\epsilon} \in M_{1}^{(0)}(\mathbb{R})$ and $\gamma^{\epsilon} \in \mathcal{C}$ such that

$$
\begin{aligned}
\sup _{\epsilon} \int_{\mathbb{R}}|x|^{2} \mathrm{~d} \gamma^{\epsilon}(0) & <\infty, \\
\lim _{\epsilon \rightarrow 0} \limsup _{\ell \rightarrow \infty} d\left(\gamma^{\ell, \epsilon}, \gamma\right) & =0, \\
\limsup _{\epsilon \rightarrow 0} \limsup _{\ell \rightarrow \infty} \widetilde{J}\left(\gamma^{\ell, \epsilon}\right) & =J_{1, \mu}(\gamma)<\infty .
\end{aligned}
$$

We proceed to state our basic local large deviations bounds.

Proposition 2.6. With $B(\gamma, \delta)$ denoting the open ball of radius $\delta>0$ centered at arbitrary $\gamma \in \mathcal{C}$, under Asmp. 1.2(a)-(c) we have the local large deviations upper bound

$$
\lim _{\delta \downarrow 0} \limsup _{N \rightarrow \infty} \frac{1}{N} \log \mathbb{P}\left(\rho^{N} \in B(\gamma, \delta)\right) \leq-I_{\iota_{\star}, \rho_{0}}(\gamma)
$$

Moreover, the sequence $\left\{\rho^{N}, N \in \mathbb{N}\right\}$ is exponentially tight in the sense that for any $M<\infty$ there exists a compact set $K_{M} \subset \mathcal{C}$, for which

$$
\limsup _{N \rightarrow \infty} \frac{1}{N} \log \mathbb{P}\left(\rho^{N} \notin K_{M}\right) \leq-M .
$$

Proposition 2.7. Under Asmp. 1.2(a)-(c) with $\iota_{\star}=1$ and $\left\{\gamma^{\ell, \epsilon}\right\} \subset \mathcal{G}$ of Prop. 2.5: (a) Asmp. 1.2(c) holds for $\rho_{0}^{\ell, \epsilon}=\gamma^{\ell, \epsilon}(0), \iota_{\star}=0$ and deterministic initial empirical measures $\left\{\rho^{N, \ell, \epsilon}(0), N \in \mathbb{N}\right\}$ such that

$$
\limsup _{\epsilon \rightarrow 0} \limsup _{\ell \rightarrow \infty} \limsup _{N \rightarrow \infty} d\left(\rho^{N}, \rho^{N, \ell, \epsilon}\right)=0 .
$$

(b) The corresponding local large deviations lower bound

$$
\liminf _{N \rightarrow \infty} \frac{1}{N} \log \mathbb{P}\left(\rho^{N, \ell, \epsilon} \in B\left(\gamma^{\ell, \epsilon}, \delta\right)\right) \geq-\widetilde{J}\left(\gamma^{\ell, \epsilon}\right)
$$

holds for any $\ell, \epsilon, \delta>0$. 
Proof of Theorem 1.4. From [10, Theorem 4.1.11] and [10, Lemma 1.2.18] we conclude that Theorem 1.4 follows once we show that for $J=J_{1, \rho_{0}}$ and any $\gamma \in \mathcal{C}$,

$$
\begin{gathered}
\lim _{\delta \downarrow 0} \limsup _{N \rightarrow \infty} \frac{1}{N} \log \mathbb{P}\left(\rho^{N} \in B(\gamma, \delta)\right) \leq-J(\gamma), \\
\lim _{\delta \downarrow 0} \liminf _{N \rightarrow \infty} \frac{1}{N} \log \mathbb{P}\left(\rho^{N} \in B(\gamma, \delta)\right) \geq-J(\gamma),
\end{gathered}
$$

and that the sequence $\left\{\rho^{N}, N \in \mathbb{N}\right\}$ is exponentially tight in the sense of (2.14). To this end, note that $\left\{\rho^{N}, N \in \mathbb{N}\right\}$ is exponentially tight (by Prop 2.6), and the upper bound (2.17) is a consequence of Prop. 2.6 and Corollary 2.3. Next, it clearly suffices to establish the lower bound (2.18) when $J(\gamma)<\infty$. To this end, fixing such $\gamma$ and $\gamma^{\ell, \epsilon} \in \mathcal{G}$ as in Prop. 2.5 (where $\mu=\rho_{0}$ ), we know from part (a) of Prop. 2.7 and (2.11) that, for any $\delta>0, \epsilon \leq \epsilon_{0}(\delta), \ell \geq \ell_{0}(\epsilon, \delta)$, if $\rho^{N, \ell, \epsilon} \in B\left(\gamma^{\ell, \epsilon}, \delta\right)$, then $\rho^{N} \in B(\gamma, 3 \delta)$ for all $N$ large enough. From part (b) of Prop. 2.7 we deduce that then

$$
\liminf _{N \rightarrow \infty} \frac{1}{N} \log \mathbb{P}\left(\rho^{N} \in B(\gamma, 3 \delta)\right) \geq-\widetilde{J}\left(\gamma^{\ell, \epsilon}\right),
$$

so using (2.12) we get (2.18) upon taking $\ell \rightarrow \infty$, then $\epsilon \downarrow 0$, and finally $\delta \downarrow 0$.

\section{Proof of Proposition 2.2 (A) And (B)}

Throughout this section, the function $A(\cdot)$ satisfies Asmp. 1.2(a), and the function $b(\cdot)$ is uniformly bounded. Then, fixing $\iota>0, \mu \in M_{1}(\mathbb{R})$, and $R=R^{(\gamma)}$ for $\gamma \in \mathcal{C}$ with $I_{\iota, \mu}(\gamma)<\infty$, we prove Prop. $2.2(\mathrm{~A})$ in a series of three lemmas, starting in Lemma 3.1 by showing that $R_{x} \in L^{3 / 2}\left(\mathbb{R}_{T}\right)$, which we improve in Lemma 3.2 to $L^{p}$ estimates on $R_{x}$ for all $\frac{3}{2} \leq p<3$. Finally, Lemma 3.3 establishes the uniform boundedness of the corresponding norms when $\mu \in M_{1}^{(0)}(\mathbb{R})$, resulting with $R_{x} \in L^{3}\left(\mathbb{R}_{T}\right)$.

Lemma 3.1. If $R=R^{(\gamma)}$ and $I_{\iota, \mu}(\gamma)=I<\infty$ for some $\mu \in M_{1}(\mathbb{R})$ then:

(a) The function $a:=A(R)$ is uniformly continuous on $\mathbb{R}_{T}$.

(b) The measure $\mathrm{d} \gamma(t) \mathrm{d} t$ on $\mathbb{R}_{T}$ has a density with respect to the Lebesgue measure on $\mathbb{R}_{T}$, whose $L^{2}$ norm restricted to any strip $S_{n, r}:=[0, T] \times\left[n-\frac{r}{4}, n+\frac{r}{4}\right]$ is bounded by a constant $C(T, I, r)<\infty$ (independent of $n \in \mathbb{Z}$ ). In particular, this density is locally square integrable, so that the weak derivative $R_{x}$ exists as a function in $L_{\text {loc }}^{2}\left(\mathbb{R}_{T}\right)$.

(c) The weak derivative $R_{x}$ is an element of $L^{3 / 2}\left(\mathbb{R}_{T}\right)$.

Proof. (a). Recall that $A(\cdot)$ is assumed to be Lipschitz, so it suffices to show uniform continuity of $R=R^{(\gamma)}$ on $\mathbb{R}_{T}$. We further assumed that $\gamma \in \mathcal{A}_{\iota, \mu}$, hence $R \in C_{b}\left(\mathbb{R}_{T}\right)$ is uniformly continuous on compact sets. In addition, the continuity of $t \mapsto \gamma(t)$ with respect to the topology of weak convergence in $M_{1}(\mathbb{R})$ implies that the image $\{\gamma(t)\}_{t \in[0, T]}$ of the compact $[0, T]$ is compact in $M_{1}(\mathbb{R})$, hence by Prokhorov's Theorem, uniformly tight. Consequently, for every $\alpha>0$, there exists finite $M=M_{\alpha}$ such that

$$
\sup _{t \in[0, T], x \geq M} \max (R(t,-x), 1-R(t, x))<\alpha,
$$

extending the uniform continuity of $R$ to all of $\mathbb{R}_{T}$.

(b). Setting hereafter $S_{n}=S_{n, 2}$, it suffices to show that the inequality

$$
\int_{S_{n}} \psi(t, x) \mathrm{d} \gamma(t) \mathrm{d} t \leq C(T, I)\|\psi\|_{2},
$$


holds for some constant $C(T, I)<\infty$, all $n \in \mathbb{Z}$, and any continuous $\psi: S_{n} \rightarrow \mathbb{R}_{+}$. Indeed, this implies the existence of the Radon-Nikodym derivative $R_{x}=\mathrm{d} \gamma(t) \mathrm{d} t / \mathrm{d} m$ on $\mathbb{R}_{T}$, whose $L^{2}\left(S_{n}\right)$-norm is bounded by $C(T, I)$ (by the same argument we used en-route to (2.6) ). Turning to prove (3.2), by definition of $I_{\iota, \mu}(\gamma)$ and the identity $\inf _{\lambda>0}\left\{\lambda y^{2}+\lambda^{-1} z^{2}\right\}=2|y z|$, we have that for $C_{1}=2\left(T I\|A\|_{\infty}\right)^{1 / 2}$ finite, $a(t, x):=$ $A(R(t, x))$, and any $g \in \overline{\mathcal{S}}$,

$$
C_{1}\left\|g_{x}\right\|_{\infty} \geq 2 I^{1 / 2}\left(\int_{\mathbb{R}_{T}}\left(g_{x}\right)^{2} a(t, x) \mathrm{d} \gamma(t) \mathrm{d} t\right)^{1 / 2} \geq \Phi_{\gamma}(g) .
$$

Considering first $n=0$, we use [26, Theorem 2] (taking there $d=1$ and $f(t, x):=$ $\psi(T-t, x))$. It provides a universal finite constant $C_{2}$ and space-time kernels $k^{\epsilon}(t, x)=$ $\epsilon^{-2} \xi(t / \epsilon) \xi(x / \epsilon), \epsilon>0$, for some infinitely differentiable, probability density $\xi(\cdot)$ of compact support, with the following property:

For any continuous $\psi: S_{0} \mapsto \mathbb{R}_{+}$there exists bounded measurable $z: \mathbb{R}_{T} \mapsto(-\infty, 0]$ (depending on $\psi$ ), non-decreasing in $t$ and supported on larger strip $S_{0,4}$, within which it is convex in $x$, such that for any $\epsilon>0$ small enough, the smooth functions $\psi^{\epsilon}=\psi * k^{\epsilon}$ and $z^{\epsilon}=z * k^{\epsilon}$ satisfy on the intermediate strip $S_{0,3}$ the inequalities

$$
\begin{aligned}
\forall c \geq 0: \quad & c^{1 / 2} \psi^{\epsilon} \leq C_{2}\left(z_{t}^{\epsilon}+c z_{x x}^{\epsilon}\right), \\
& \frac{1}{2}\left|z_{x}^{\epsilon}\right| \leq-z^{\epsilon} \leq C_{2}\|\psi\|_{2} .
\end{aligned}
$$

In the preceding, the compact support of $z$ is specified in the proof of [26, Theorem 2] after [26, display (29)].

Picking a $[0,1]$-valued truncation function $\phi \in \overline{\mathcal{S}}$, supported on $S_{0,3}$ with $\phi \equiv 1$ on $S_{0,2}$, we note that for each $\epsilon>0$ as above, the non-negative function $g=-z^{\epsilon} \phi$ is in $\overline{\mathcal{S}}$, supported on $S_{0,3}$ such that by (3.5) both $\|g\|_{\infty}$ and $\left\|g_{x}\right\|_{\infty}$ are bounded by $C_{3}\|\psi\|_{2}$ (for the universal constant $\left.C_{3}=\left(2+\left\|\phi_{x}\right\|_{\infty}\right) C_{2}\right)$. Consequently, applying the bound (3.3) for such choice of $g$, we deduce that

$$
C_{1} C_{3}\|\psi\|_{2} \geq \Phi_{\gamma}(g) \geq-\int_{S_{0,3}}\left(g_{t}+a g_{x x}\right) \mathrm{d} \gamma(t) \mathrm{d} t-T\|b(R)\|_{\infty}\left\|g_{x}\right\|_{\infty}-\|g(0, \cdot)\|_{\infty} .
$$

Next with $\phi_{t}, \phi_{x}$ and $\phi_{x x}$ uniformly bounded by some universal constant $C_{4}$, it follows from Leibniz's rule and (3.5) that

$$
\left|\left(g_{t}+a g_{x x}\right)+\left(z_{t}^{\epsilon}+a z_{x x}^{\epsilon}\right) \phi\right|=\left|z^{\epsilon}\left(\phi_{t}+a \phi_{x x}\right)+2 a \phi_{x} z_{x}^{\epsilon}\right| \leq C_{2} C_{4}\left(1+5\|a\|_{\infty}\right)\|\psi\|_{2},
$$

out of which we deduce by simple algebra that

$$
C_{5}\|\psi\|_{2} \geq \int_{S_{0,3}}\left(z_{t}^{\epsilon}+a z_{x x}^{\epsilon}\right) \phi \mathrm{d} \gamma(t) \mathrm{d} t
$$

for finite $C_{5}$ depending only on $T,\|b\|_{\infty},\|A\|_{\infty}$ and the constants $C_{i}, i=1, \ldots, 4$. With $z$ non-decreasing in $t$, so are $z^{\epsilon}=z * k^{\epsilon}$. Further, as $\xi(\cdot)$ has compact support, the convexity of $z$ in $x$ within $S_{0,4}$ implies that $z^{\epsilon}$ is convex in $x$ on $S_{0,3}$, provided $\epsilon>0$ is small enough. Thus, both $z_{t}^{\epsilon}$ and $z_{x x}^{\epsilon}$ are non-negative, so considering (3.4) for the strictly positive $c=\underline{a}$ of Asmp. 1.2(a) and recalling that $\phi \equiv 1$ on $S_{0}=S_{0,2}$ (with $\phi \geq 0$ elsewhere), we have from (3.6) that

$$
\begin{array}{r}
C_{2} C_{5}\|\psi\|_{2} \geq C_{2} \int_{S_{0}}\left(z_{t}^{\epsilon}+a z_{x x}^{\epsilon}\right) \phi \mathrm{d} \gamma(t) \mathrm{d} t \geq \int_{S_{0}} C_{2}\left(z_{t}^{\epsilon}+c z_{x x}^{\epsilon}\right) \mathrm{d} \gamma(t) \mathrm{d} t \\
\geq c^{1 / 2} \int_{S_{0}} \psi^{\epsilon} \mathrm{d} \gamma(t) \mathrm{d} t .
\end{array}
$$


With $\psi \in C_{b}\left(S_{0}\right)$, clearly $\psi^{\epsilon} \rightarrow \psi$ uniformly on $S_{0}$ when $\epsilon \downarrow 0$, leading to (3.2) for $n=0$ and the universal finite constant $C(T, I)=C_{2} C_{5} c^{-1 / 2}$ which depends only on $T, I$, and the functions $b(\cdot)$ and $A(\cdot)$ on $[0,1]$.

To extend (3.2) to other values of $n \in \mathbb{Z}$, let $\mu_{n}(\cdot):=\mu(\cdot+n)$ and note that the path $\gamma_{n}(\cdot, \cdot)=\gamma(\cdot, \cdot+n) \in \mathcal{A}_{\iota, \mu_{n}}$ if and only if $\gamma \in \mathcal{A}_{\iota, \mu}$. Setting next $g_{n}(\cdot, \cdot)=g(\cdot, \cdot+n)$ we clearly have that $\left(\gamma_{n}, \mathcal{R}^{\gamma_{n}} g_{n}\right)(s)$ is independent of $n$ for each $s \in[0, T]$. Hence, $I_{\iota, \mu_{n}}\left(\gamma_{n}\right)=I$ and, since to any non-negative $\psi \in C_{b}\left(S_{n}\right)$ corresponds non-negative $\psi_{n}(\cdot, \cdot)=\psi(\cdot, \cdot+n) \in C_{b}\left(S_{0}\right)$, by the preceding proof:

$$
\int_{S_{n}} \psi(t, x) \mathrm{d} \gamma(t) \mathrm{d} t=\int_{S_{0}} \psi_{n}(t, x) \mathrm{d} \gamma_{n}(t) \mathrm{d} t \leq C(T, I)\left\|\psi_{n}\right\|_{2}=C(T, I)\|\psi\|_{2}
$$

(as $C$ is independent of the initial measure). This completes the proof of part (b).

(c). Fixing $n \in \mathbb{Z}$ we already know that $R_{x} \in L^{p}\left(S_{n}\right)$ for $p=1,2$. Further, upon applying Cauchy-Schwarz inequality with respect to Lebesgue measure on $S_{n}$, we have that

$$
\int_{S_{n}} R_{x}^{3 / 2} \mathrm{~d} m \leq\left(\int_{S_{n}} R_{x} \mathrm{~d} m\right)^{1 / 2}\left(\int_{S_{n}} R_{x}^{2} \mathrm{~d} m\right)^{1 / 2} .
$$

We have shown in part (b) that the right-most term is bounded uniformly in $n$, so our claim that the left-side is summable over $n \in \mathbb{Z}$ follows from finiteness of $\sum_{|n| \geq 1} \kappa_{n}$, with $\kappa_{n}:=\left(\int_{S_{n}} R_{x} \mathrm{~d} m\right)^{1 / 2}$. Next, taking $\iota>0$ as in the given moment condition (1.6), we get by Cauchy-Schwarz that for $c_{1}:=\sum_{|n| \geq 1}|n|^{-(1+\iota)}$ and $c_{2}:=\sup \left\{|n / y|^{1+\iota}: y \in\right.$ $\left.S_{n},|n| \geq 1\right\}$ finite,

$$
\begin{aligned}
\left(\sum_{|n| \geq 1} \kappa_{n}\right)^{2} \leq c_{1} \sum_{|n| \geq 1}|n|^{1+\iota} \kappa_{n}^{2} & \leq c_{1} c_{2} \sum_{|n| \geq 1} \int_{S_{n}}|y|^{1+\iota} R_{x}(t, y) \mathrm{d} m(t, y) \\
& \leq c_{1} c_{2} \int_{\mathbb{R}_{T}}|y|^{1+\iota} \mathrm{d} \gamma(t) \mathrm{d} t<\infty
\end{aligned}
$$

(see (1.6) for the right-most inequality).

Lemma 3.2. If $R=R^{(\gamma)}$ and $I_{\iota, \mu}(\gamma)<\infty$, then $R_{x}$ exists as element of $L^{p}\left(\mathbb{R}_{T}\right)$ for all $\frac{3}{2} \leq p<3$.

Proof. We fix $\frac{3}{2} \leq p<3$ and set $\frac{3}{2}<q \leq 3$ such that $\frac{1}{p}+\frac{1}{q}=1$. For any given non-negative $f \in \overline{\mathcal{S}}$, we consider the backward Cauchy problem

$$
u_{t}+a(t, x) u_{x x}+f(t, x) u=0, \quad u(T, \cdot)=1
$$

with $a(t, x):=A(R(t, x))$. While $a(t, x) \in C_{b}\left(\mathbb{R}_{T}\right)$ is not differentiable in $x$, recall part (a) of Lemma 3.1 that it is uniformly continuous and bounded away from zero. Considering [27, Theorem 2.1] for $w:=u-1$, such $a(\cdot, \cdot)$ and any $f \in L^{r}\left(\mathbb{R}_{T}\right)$, we see that (3.7) has a weak solution, for which $w=u-1$ is an element of $W_{r}^{1,2}\left(\mathbb{R}_{T}\right)$. Further, here $f \in L^{q}\left(\mathbb{R}_{T}\right) \cap L^{2}\left(\mathbb{R}_{T}\right) \cap L^{3}\left(\mathbb{R}_{T}\right)$ from which it follows that the norm bounds of [27, Theorem 4.1] can be refined to an estimate on the norms in $L^{q}\left(\mathbb{R}_{T}\right) \cap L^{2}\left(\mathbb{R}_{T}\right) \cap L^{3}\left(\mathbb{R}_{T}\right)$. From the latter estimate, we conclude upon applying the method of continuity (see e.g. [30, section III.2]), that for $f \in \overline{\mathcal{S}}$ the problem (3.7) has a weak solution, for which $u-1$ is an element of $W_{q}^{1,2}\left(\mathbb{R}_{T}\right) \cap W_{2}^{1,2}\left(\mathbb{R}_{T}\right) \cap W_{3}^{1,2}\left(\mathbb{R}_{T}\right)$. Next, let $Y(\cdot)$ denotes the canonical process having the law $P$ of a diffusion with generator $a(t, x) \frac{\mathrm{d}^{2}}{\mathrm{~d} x^{2}}$ (which exists thanks to uniform continuity and boundedness from above and below of $a$ ). Applying Itô's formula in the form of the last identity in [25, chapter 10, Theorem 1] 
(again using the boundedness from above and below of $a$ ), we obtain the stochastic representation of such weak solution of (3.7),

$$
u(t, x)=\mathbb{E}^{P}\left[\exp \left(\int_{t}^{T} f(s, Y(s)) \mathrm{d} s\right) \mid Y(t)=x\right] .
$$

The latter representation implies that $u \geq 1$, and by Portenko's Lemma (see [35, inequality (6)], note that it only relies on the standard heat kernel estimate for the diffusion with law $P$ and that $q>\frac{3}{2}$ throughout this proof), there is a non-decreasing function $G_{q}:[0, \infty) \rightarrow[1, \infty)$ depending only on $\frac{3}{2}<q \leq 3$ (and not on $f$ ), such that

$$
1 \leq u(t, x) \leq G_{q}\left(\|f\|_{L^{q}\left(\mathbb{R}_{T}\right)}\right), \quad(t, x) \in \mathbb{R}_{T} .
$$

Next, observe that the non-negative $v:=\log u$ inherits the bound

$$
0 \leq \mathrm{v}(t, x) \leq G_{q}\left(\|f\|_{L^{q}\left(\mathbb{R}_{T}\right)}\right), \quad(t, x) \in \mathbb{R}_{T} .
$$

Recall that $u_{t}, u_{x x} \in L^{3}\left(\mathbb{R}_{T}\right)$, and with $u \in W_{2}^{1,2}\left(\mathbb{R}_{T}\right)$ further $u_{x} \in L^{6}\left(\mathbb{R}_{T}\right)$ (by the parabolic Sobolev inequality in the form of [29, chapter II, Lemma 3.3]), which for positive $u$ bounded away from zero, imply also that $\mathrm{v}_{t}, \mathrm{v}_{x x} \in L^{3}\left(\mathbb{R}_{T}\right)$ and $\mathrm{v}_{x} \in L^{6}\left(\mathbb{R}_{T}\right)$ (with $\mathrm{v}_{x x}+\mathrm{v}_{x}^{2}=u_{x x} / u$ ). From this it follows in turn that $m$-a.e. on $\mathbb{R}_{T}$ such $\vee$ and its generalized derivatives, satisfy the backward equation

$$
\mathrm{v}_{t}+a(t, x) \mathrm{v}_{x x}+a(t, x) \mathrm{v}_{x}^{2}+f(t, x)=0, \quad \mathrm{v}(T, \cdot)=0 .
$$

Recall that while proving Corollary 2.3 we found in (2.8) a function $h$ whose $L^{2}\left(\mathbb{R}_{T}, a R_{x} \mathrm{~d} m\right.$ )-norm is bounded by $C_{6}:=2 I^{1 / 2}+\|b\|_{\infty}(T / \underline{a})^{1 / 2}$ (due to (2.6) and the assumed bounds on $b(\cdot)$ and $A(\cdot) \geq \underline{a}$ ), so that, for any $g \in \overline{\mathcal{S}}$,

$$
(\gamma, g)(T)-(\gamma, g)(0)=\int_{\mathbb{R}_{T}}\left(g_{t}+a g_{x x}-a g_{x} h\right) R_{x} \mathrm{~d} m
$$

(consider (2.7)). With $R_{x} \in L^{3 / 2}\left(\mathbb{R}_{T}\right)$ (by part (c) of Lemma 3.1), and Hölder's inequality

$$
\int_{\mathbb{R}_{T}}|\varphi| R_{x} \mathrm{~d} m \leq\left(\int R_{x}^{3 / 2} \mathrm{~d} m\right)^{2 / 3}\left(\int|\varphi|^{3} \mathrm{~d} m\right)^{1 / 3},
$$

we deduce that $\mathrm{v}_{t}, a \mathrm{v}_{x x}$ and $a \mathrm{v}_{x}^{2}$ are integrable with respect to $R_{x} \mathrm{~d} m$ (as is $a h^{2}$ ). Thus, with $v$ bounded, we get from (3.11) upon approximating $v$ in suitable (mixed)-norm by functions $g_{k} \in \overline{\mathcal{S}}$ that

$$
(\gamma, \mathrm{v})(T)-(\gamma, \mathrm{v})(0)=\int_{\mathbb{R}_{T}}\left(\mathrm{v}_{t}+a \mathbf{v}_{x x}-a \mathbf{v}_{x} h\right) R_{x} \mathrm{~d} m
$$

The latter identity, in combination with (3.10), results with

$$
\begin{aligned}
\int_{\mathbb{R}_{T}} f R_{x} \mathrm{~d} m & =-\int_{\mathbb{R}_{T}}\left(\mathrm{v}_{x} h+\mathrm{v}_{x}^{2}\right) a R_{x} \mathrm{~d} m+(\gamma, \mathrm{v})(0) \\
& \leq \frac{1}{4} \int_{\mathbb{R}_{T}} h^{2} a R_{x} \mathrm{~d} m+\sup _{(t, x) \in \mathbb{R}_{T}} \mathrm{v}(t, x) \leq \frac{1}{4} C_{6}^{2}+G_{q}\left(\|f\|_{L^{q}\left(\mathbb{R}_{T}\right)}\right),
\end{aligned}
$$

where the first inequality is merely the non-negativity of $L^{2}\left(\mathbb{R}_{T}, a R_{x} \mathrm{~d} m\right)$-norm of $\mathrm{v}_{x}+$ $h / 2$, and the second follows from (3.9). With $C_{6}$ independent of $f$, we have that the linear functional $f \mapsto \int_{\mathbb{R}_{T}} f R_{x} \mathrm{~d} m$ is bounded on $\overline{\mathcal{S}}$ with respect to the $L^{q}\left(\mathbb{R}_{T}\right)$-norm, hence $R_{x} \in L^{p}\left(\mathbb{R}_{T}\right)$ for $1 / p=1-1 / q$, as claimed.

Lemma 3.3. Suppose $R=R^{(\gamma)}$ and $I_{\iota, \mu}(\gamma)=I<\infty$ for some $\mu \in M_{1}^{(0)}(\mathbb{R})$. Then $R_{x} \in L^{3}\left(\mathbb{R}_{T}\right)$. 
Proof. Recall from Lemma 3.2 that $R_{x} \in L^{p}\left(\mathbb{R}_{T}\right)$ for any $p \in[3 / 2,3)$. Hence, $R_{x} \in L^{3}\left(\mathbb{R}_{T}\right)$, provided $p \mapsto\left\|R_{x}\right\|_{L^{p}\left(\mathbb{R}_{T}\right)}$ is uniformly bounded over such $p$, as we prove here.

Step 1. Recall that $a(t, x)=A(R)$ and $\int_{\mathbb{R}_{T}}\left(g_{t} R_{x}+g_{x t} R\right) \mathrm{d} m=0$ (integration by parts in $x$ ). When substituted in (3.11), these yield that the path of CDF-s $R=R^{(\gamma)}$ is a generalized solution of the Cauchy problem

$$
R_{t}-\left(A(R) R_{x}\right)_{x}=h A(R) R_{x}, \quad R(0, \cdot)=F_{\mu}
$$

with respect to the collection $\overline{\mathcal{S}}_{x}:=\left\{g_{x}: g \in \overline{\mathcal{S}}\right\}$ of test functions, for some function $h$ whose $L^{2}\left(\mathbb{R}_{T}, a R_{x} \mathrm{~d} m\right)$-norm is bounded by finite $C_{6}=C_{6}(T, I)$. That is,

$$
\int_{\mathbb{R}}\left(g_{x} R\right)(T, x) \mathrm{d} x-\int_{\mathbb{R}}\left(g_{x} R\right)(0, x) \mathrm{d} x=\int_{\mathbb{R}_{T}}\left(g_{x t} R-g_{x x} a R_{x}+g_{x} h a R_{x}\right) \mathrm{d} m,
$$

for any $g \in \overline{\mathcal{S}}$.

Next, applying for $f=A(R) R_{x}$ the Hölder's inequality in the form of

$$
\int_{\mathbb{R}_{T}}|h|^{q} f^{q} \mathrm{~d} m \leq\left(\int_{\mathbb{R}_{T}}|h|^{2} f \mathrm{~d} m\right)^{q / 2}\left(\int_{\mathbb{R}_{T}} f^{p} \mathrm{~d} m\right)^{q /(2 p)}
$$

with $\frac{3}{2} \leq p<3$ and $\frac{q}{2}+\frac{q}{2 p}=1$, namely $q:=\frac{2 p}{p+1} \in[6 / 5,3 / 2)$, we deduce that $h A(R) R_{x} \in L^{q}\left(\mathbb{R}_{T}\right)$. Thus, by the regularity theory for the heat equation (see inequalities (3.1) and (3.2) in [29, chapter IV], or [27, Theorem 2.1]), we have that the function

$$
V(t, x):=\int_{0}^{t} \int_{\mathbb{R}}\left(h A(R) R_{x}\right)(s, y) \varphi(t-s, x-y) \mathrm{d} y \mathrm{~d} s,
$$

obtained by convolving with the heat kernel $\varphi(t, x)=(4 \pi t)^{-1 / 2} \exp \left(-x^{2} / 4 t\right)$, is a generalized solution of the auxiliary Cauchy problem

$$
V_{t}-V_{x x}=h A(R) R_{x}, \quad V(0, \cdot)=0
$$

(with respect to the collection $\overline{\mathcal{S}}_{x}$ ). Further,

$$
\|V\|_{W_{q}^{1,2}\left(\mathbb{R}_{T}\right)} \leq C_{7}\left\|h A(R) R_{x}\right\|_{L^{q}\left(\mathbb{R}_{T}\right)}
$$

where $C_{7}<\infty$ is a uniform constant (which in particular does not depend on $q$ as long as $q$ belongs to a compact interval). Thus, $V \in W_{q}^{1,2}\left(\mathbb{R}_{T}\right)$ and, due to the parabolic Sobolev inequality (in the form of [29, chapter II, Lemma 3.3]), also $V, V_{x} \in L^{r}\left(\mathbb{R}_{T}\right.$ ) for any $q \leq r \leq p^{\prime}$ where $p^{\prime}:=\left(\frac{1}{q}-\frac{1}{3}\right)^{-1}=\frac{6 p}{3+p}$. Further, the constants in this parabolic Sobolev inequality can be chosen uniformly over $p$ in any given compact interval, hence for some uniform $C_{8}<\infty$ and all $p \in[3 / 2,3)$,

$$
\left\|V_{x}\right\|_{L^{p^{\prime}\left(\mathbb{R}_{T}\right)}} \leq C_{8}\left\|h A(R) R_{x}\right\|_{L^{q}\left(\mathbb{R}_{T}\right)}
$$

where $q=\frac{2 p}{p+1}$ and $p^{\prime}=\frac{6 p}{3+p}$. Similarly, the function

$$
Z(t, x):=\int_{\mathbb{R}} F_{\mu}(y) \varphi(t, x-y) \mathrm{d} y,
$$

is a classical solution of the initial value problem

$$
Z_{t}-Z_{x x}=0, \quad Z(0, \cdot)=F_{\mu} .
$$


Since $F_{\mu}(y)=\int_{-\infty}^{y} \theta(z) \mathrm{d} z$ for $\theta:=\frac{\mathrm{d} \mu}{\mathrm{d} x}$, clearly $Z_{x}$ is given by the convolution (in space) of $\theta$ and the heat kernel $\varphi(t, \cdot)$. Consequently, by Fubini's theorem and Young's inequality, for any $p, q \geq 1$ such that $1 / p+1 / q=1 / r+1$,

$$
\left\|Z_{x}\right\|_{L^{r}\left(\mathbb{R}_{T}\right)}^{r}=\int_{0}^{T}\left\|Z_{x}(t, \cdot)\right\|_{L^{r}(\mathbb{R})}^{r} \mathrm{~d} t \leq\|\theta\|_{L^{q}(\mathbb{R})}^{r} \int_{0}^{T}\|\varphi(t, \cdot)\|_{L^{p}(\mathbb{R})}^{r} \mathrm{~d} t .
$$

By assumption $\mu \in M_{1}^{(0)}(\mathbb{R})$, so $\theta \in L^{1}(\mathbb{R}) \cap L^{q_{0}}(\mathbb{R})$ for some $q_{0}>1$, and hence the norms $\|\theta\|_{L^{q}(\mathbb{R})}, 1 \leq q \leq q_{0}$ are uniformly bounded. Further, $\|\varphi(t, \cdot)\|_{L^{p}(\mathbb{R})} \leq t^{-(p-1) /(2 p)}$ for all $t>0$ and $p \geq 1$. Considering $q=q_{0} \wedge r$, with $p \geq 1$ such that for $1 \leq r \leq 3$ the value of $r(p-1) /(2 p)$ is bounded away from one, we conclude that $\left\|Z_{x}\right\|_{L^{r}\left(\mathbb{R}_{T}\right)}$, $1 \leq r \leq 3$ are uniformly (in $r$ ) bounded by some finite $C_{9}=C_{9}\left(T,\|\theta\|_{q_{0}}\right)$.

From (3.12), (3.15), and (3.17) it follows by a direct computation that $U:=R-V-Z$ is a generalized solution of the Cauchy problem

$$
U_{t}-\left(A(R) U_{x}\right)_{x}=\left[(A(R)-1) V_{x}+(A(R)-1) Z_{x}\right]_{x}, \quad U(0, \cdot)=0,
$$

with respect to test functions in $\overline{\mathcal{S}}_{x}$ (interpreted via integration by parts in $t$ and $x$, similarly to what we have done in (3.13) ).

Step 2. We proceed to obtain the uniform in $p$ bounds on $L^{p}$-norms of $U_{x}$ (and thereby those of $R_{x}$ ) out of the bounds we already have for $V_{x}$ and $Z_{x}$. To this end, recalling part (a) of Lemma 3.1, refining the norm estimates in [27, Theorem 6.2] to estimates in $L^{p}\left(\mathbb{R}_{T}\right) \cap L^{p^{\prime}}\left(\mathbb{R}_{T}\right)$ with $p^{\prime}=\frac{6 p}{3+p}$ and applying the method of continuity (see e.g. [30, section III.2]), we deduce the existence of a solution $\hat{U}$ of the problem (3.18) in the space $\mathcal{H}_{p}(T) \cap \mathcal{H}_{p^{\prime}}(T)$ defined in [27]. In particular, $\hat{U} \in L^{p^{\prime}}\left(\mathbb{R}_{T}\right), \hat{U}_{x} \in L^{p^{\prime}}\left(\mathbb{R}_{T}\right)$ and

$$
\|\hat{U}\|_{L^{p^{\prime}}\left(\mathbb{R}_{T}\right)}+\left\|\hat{U}_{x}\right\|_{L^{p^{\prime}\left(\mathbb{R}_{T}\right)}} \leq C_{10}\left(\left\|V_{x}\right\|_{L^{p^{\prime}\left(\mathbb{R}_{T}\right)}}+\left\|Z_{x}\right\|_{L^{p^{\prime}\left(\mathbb{R}_{T}\right)}}\right)
$$

with $p^{\prime}=\frac{6 p}{3+p}$ and where the finite constant $C_{10}=C_{10}\left(T,\|A\|_{\infty}, \underline{a}\right)$ can be chosen uniformly for all $2 \leq p^{\prime}<3$.

We show in Step 3 below that $U=\hat{U}$ Lebesgue a.e. on $\mathbb{R}_{T}$. Thus, $U_{x} \in L^{p^{\prime}}\left(\mathbb{R}_{T}\right)$ and consequently also $R_{x}=U_{x}+V_{x}+Z_{x} \in L^{p^{\prime}}\left(\mathbb{R}_{T}\right)$. Hence, combining the norm bounds (3.16) and (3.19) with Hölder's inequality (3.14), yields the estimate

$$
\left\|R_{x}\right\|_{L^{p^{\prime}\left(\mathbb{R}_{T}\right)}} \leq C_{11}\left\|R_{x}\right\|_{L^{p}\left(\mathbb{R}_{T}\right)}^{1 / 2}+C_{12}
$$

for the finite constants $C_{11}=\left(1+C_{10}\right) C_{8}\left(C_{6}\|A\|_{\infty}\right)^{1 / 2}$ and $C_{12}=\left(1+C_{10}\right) C_{9}$ (both independent of $\frac{3}{2} \leq p<3$ ). Since $p^{\prime} \geq p>1$, by Jensen's inequality (for the convex function $x^{\left(p^{\prime}-1\right) /(p-1)}$ and the probability measure $T^{-1} R_{x} \mathrm{~d} m$ on $\left.\mathbb{R}_{T}\right)$, we deduce that for any $p \in[3 / 2,3)$

$$
\left\|R_{x}\right\|_{L^{p}\left(\mathbb{R}_{T}\right)}^{r(p)} \leq C_{13}\left\|R_{x}\right\|_{L^{p^{\prime}}\left(\mathbb{R}_{T}\right)},
$$

where $r(p)=\frac{p\left(p^{\prime}-1\right)}{p^{\prime}(p-1)} \geq 1$ and $C_{13}=\max \left(1, T^{1 / 2}\right)$ is finite. Combining this with (3.20) we conclude that

$$
\left\|R_{x}\right\|_{L^{p}\left(\mathbb{R}_{T}\right)} \leq \max \left\{1, C_{13}\left(C_{11}\left\|R_{x}\right\|_{L^{p}\left(\mathbb{R}_{T}\right)}^{1 / 2}+C_{12}\right)\right\} .
$$

As explained before, having $\left\|R_{x}\right\|_{L^{p}\left(\mathbb{R}_{T}\right)}$ bounded, uniformly over $\frac{3}{2} \leq p<3$, yields that $R_{x} \in L^{3}\left(\mathbb{R}_{T}\right)$ (whose $L^{3}$-norm is bounded by some $C(T, I)$ finite).

Step 3. Turning to show that Lebesgue a.e. $U=\hat{U}$ on $\mathbb{R}_{T}$, we start by verifying that $R-Z \in L^{p}\left(\mathbb{R}_{T}\right)$. Indeed, as $|R-Z| \leq 1$, it suffices to check this for $p=1$, in which 
case by the triangle inequality,

$$
\begin{aligned}
\int_{\mathbb{R}_{T}}|R-Z| \mathrm{d} m & \leq \int_{\mathbb{R}_{T}^{+}}(1-R) \mathrm{d} m+\int_{\mathbb{R}_{T}^{+}}(1-Z) \mathrm{d} m+\int_{\mathbb{R}_{T}^{-}} R \mathrm{~d} m+\int_{\mathbb{R}_{T}^{-}} Z \mathrm{~d} m \\
& =\int_{\mathbb{R}_{T}}|x| R_{x} \mathrm{~d} m+\int_{\mathbb{R}_{T}}|x| Z_{x} \mathrm{~d} m,
\end{aligned}
$$

where $\mathbb{R}_{T}^{+}=[0, T] \times \mathbb{R}_{+}, \mathbb{R}_{T}^{-}=[0, T] \times \mathbb{R}_{-}$and the last equality applies since $R(t, \cdot)$ and $Z(t, \cdot)$ are CDF-s having densities $R_{x}$ and $Z_{x}$ with respect to Lebesgue measure on $\mathbb{R}_{T}$. Since $\gamma \in \mathcal{A}_{\iota, \mu}$ the first term on the right-side of (3.21) is finite (see (1.6)), whereas the second term amounts to $\int_{0}^{T} \mathbf{E}[|Y+W(2 t)|] \mathrm{d} t$ for $Y$ of law $\mu$, independent of the standard Brownian motion $\{W\}$. By the triangle inequality, the latter term is at most $\frac{1}{3}(2 T)^{3 / 2}+T \int_{\mathbb{R}}|x| \mathrm{d} \mu$, hence finite (since $\left.\mu \in M_{1}^{(0)}(\mathbb{R})\right)$.

Now, with $R-Z \in L^{p}\left(\mathbb{R}_{T}\right)$, and having seen already in Step 1 that $V \in L^{p}\left(\mathbb{R}_{T}\right)$ (by the parabolic Sobolev inequality for $\left.V \in W_{q}^{1,2}\left(\mathbb{R}_{T}\right)\right)$, we conclude that $U=R-Z-V \in$ $L^{p}\left(\mathbb{R}_{T}\right)$. Similarly, $U_{x} \in L^{p}\left(\mathbb{R}_{T}\right)$, since $R_{x} \in L^{p}\left(\mathbb{R}_{T}\right)$ (from Lemma 3.2), and we have already established in Step 1 that $Z_{x} \in L^{p}\left(\mathbb{R}_{T}\right)$ and $V_{x} \in L^{p}\left(\mathbb{R}_{T}\right)$.

Recall that $h \in L^{1}\left(R_{x} \mathrm{~d} m\right)$, so $V \in L^{1}\left(\mathbb{R}_{T}\right)$, and hence $U \in L^{1}\left(\mathbb{R}_{T}\right)$ as well. Now, fixing $g \in \overline{\mathcal{S}}$ we let

$$
f(t, x)=-\int_{x}^{\infty} g(t, y) \mathrm{d} y, \quad(t, x) \in \mathbb{R}_{T}
$$

and claim that, for Lebesgue almost every $0 \leq t_{1}<t_{2} \leq T$,

$$
\begin{aligned}
\int_{\mathbb{R}} f\left(t_{2}, x\right) U\left(t_{2}, x\right) \mathrm{d} x-\int_{\mathbb{R}} f\left(t_{1}, x\right) U\left(t_{1}, x\right) \mathrm{d} x-\int_{t_{1}}^{t_{2}} \int_{\mathbb{R}} U f_{t} \mathrm{~d} m \\
=-\int_{t_{1}}^{t_{2}} \int_{\mathbb{R}}\left[A(R) U_{x}+(A(R)-1)\left(V_{x}+Z_{x}\right)\right] f_{x} \mathrm{~d} m .
\end{aligned}
$$

Indeed, from the weak formulation of the PDE in (3.18) we have (3.23) when $f \in \overline{\mathcal{S}}_{x}$. This in turn extends to all $f$ as in (3.22), by the uniform joint approximation on compacts of the continuously differentiable and bounded $\left(f, f_{t}, f_{x}\right)$ by $\left(\widetilde{g}, \widetilde{g}_{t}, \widetilde{g}_{x}\right)$ for some $\widetilde{g} \in \overline{\mathcal{S}}_{x}$ (taking such compact large enough that the contribution to (3.23) from its complement be as small as desired, which we can do since $A(R) U_{x}+(A(R)-1)\left(V_{x}+\right.$ $\left.Z_{x}\right) \in L^{p}\left(\mathbb{R}_{T}\right)$ and $\left.U \in L^{1}\left(\mathbb{R}_{T}\right)\right)$. Next, integrating by parts in space on the left side of (3.23), we conclude that the function $W(t, x)=\int_{-\infty}^{x} U(t, y) \mathrm{d} y$ is a generalized solution of the Cauchy problem

$$
W_{t}=A(R) W_{x x}+(A(R)-1)\left(V_{x}+Z_{x}\right), \quad W(0, \cdot)=0
$$

on $\mathbb{R}_{T}$ (with respect to test functions from $\overline{\mathcal{S}}$ ). This, $W_{x x}=U_{x} \in L^{p}\left(\mathbb{R}_{T}\right), V_{x} \in L^{p}\left(\mathbb{R}_{T}\right)$, and $Z_{x} \in L^{p}\left(\mathbb{R}_{T}\right)$ imply $W_{t} \in L^{p}\left(\mathbb{R}_{T}\right)$. Thus, in view of [27, norm estimate (6.1)], we have that $U \in \mathcal{H}_{p}\left(\mathbb{R}_{T}\right)$, so that $U=\hat{U}$ Lebesgue almost everywhere by [27, Theorem 2.4].

Proof of Proposition 2.2 (B). From the proof of Lemma 3.3 we recall that $R-$ $Z, Z_{x}, R_{x} \in L^{r}\left(\mathbb{R}_{T}\right)$ for all $r \in[1,3]$ and $V \in W_{q}^{1,2}\left(\mathbb{R}_{T}\right)$ for all $q \in[6 / 5,3 / 2]$ (where, since $R_{x} \in L^{3}\left(\mathbb{R}_{T}\right)$, the argument leading to (3.16) now applies also for $p=3$ and $q=3 / 2)$. Consequently, $V_{t}, V_{x x} \in L^{q}\left(\mathbb{R}_{T}\right)$ for any $q \in[6 / 5,3 / 2]$ and, by the parabolic Sobolev inequality (in the form of [29, chapter II, Lemma 3.3]), also $V, V_{x} \in L^{p^{\prime}}\left(\mathbb{R}_{T}\right)$ for any $p^{\prime}=\left(\frac{1}{q}-\frac{1}{3}\right)^{-1} \in[3 / 2,3]$. Since $U=R-Z-V$, this in turn implies that $U, U_{x} \in L^{p}\left(\mathbb{R}_{T}\right)$ for all $p \in[3 / 2,3]$. Further, $Z_{t}=Z_{x x} \in L^{q}\left(\mathbb{R}_{T}\right)$ for all $q \in[6 / 5,3 / 2]$, 
hence it suffices to show that $U \in W_{q}^{1,2}\left(\mathbb{R}_{T}\right)$, as then $U_{t}, U_{x x} \in L^{q}\left(\mathbb{R}_{T}\right)$ implying the same for $R_{t}$ and $R_{x x}$. To this end, rewriting (3.18) we have that $U$ solves

$$
U_{t}-A(R) U_{x x}=A^{\prime}(R) R_{x} U_{x}+\left[(A(R)-1) V_{x}+(A(R)-1) Z_{x}\right]_{x}
$$

with respect to test functions in $\overline{\mathcal{S}}_{x}$, starting at $U(0, \cdot)=0$. For any $q \in[6 / 5,3 / 2]$ there exist $r, p \in[3 / 2,3]$ such that $\frac{1}{p}+\frac{1}{r}=\frac{1}{q}$, so with $A$ and $A^{\prime}$ bounded, by Hölder's inequality and the preceding integrability properties, the right side in (3.25) belongs to $L^{q}\left(\mathbb{R}_{T}\right)$ for all $q \in[6 / 5,3 / 2]$. Thus, fixing $q \in[6 / 5,3 / 2]$ we may apply [27, Theorem 2.1] to deduce that there is a function $\tilde{U} \in W_{q}^{1,2}\left(\mathbb{R}_{T}\right) \cap W_{3 / 2}^{1,2}\left(\mathbb{R}_{T}\right)$ which satisfies

$$
\tilde{U}_{t}-A(R) \tilde{U}_{x x}=A^{\prime}(R) R_{x} U_{x}+\left[(A(R)-1) V_{x}+(A(R)-1) Z_{x}\right]_{x}
$$

and $\tilde{U}(0, \cdot)=0$. In particular, $\tilde{U}, \tilde{U}_{x} \in L^{3}\left(\mathbb{R}_{T}\right)$. Now, we let $\phi_{k}, k \in \mathbb{N}$ be a truncation sequence such that $\phi_{k} \in C^{\infty}(\mathbb{R}), 0 \leq \phi_{k} \leq 1, \phi_{k} \equiv 1$ on $[-k, k], \phi_{k} \equiv 0$ on $(-\infty,-k-$ 1] $\cup[k+1, \infty)$ and $\max \left(\left|\phi_{k}^{\prime}\right|,\left|\phi_{k}^{\prime \prime}\right|\right) \leq 2$. Next, fixing $k \in \mathbb{N}$ we set $\tilde{\Delta}=\phi_{k}(U-\tilde{U})$. Then, $\tilde{\Delta}$ is a generalized solution of the problem

$$
\tilde{\Delta}_{t}-\left(A(R) \tilde{\Delta}_{x}\right)_{x}+A^{\prime}(R) R_{x} \tilde{\Delta}_{x}=\tilde{\psi}_{k}, \quad \Delta(0, \cdot)=0
$$

with respect to test functions in $\overline{\mathcal{S}}_{x}$, where

$$
\tilde{\psi}_{k}=-A(R) \phi_{k}^{\prime \prime}(U-\tilde{U})-2 A(R) \phi_{k}^{\prime}(U-\tilde{U})_{x}
$$

is in $L^{3}\left(\mathbb{R}_{T}\right)$. Further, $\tilde{\psi}_{k}(t, x)=0$ for all $x \in(-k, k)$, so by our choice of $\phi_{k}$,

$$
\lim _{k \rightarrow \infty}\left\|\tilde{\psi}_{k}\right\|_{L^{3}\left(\mathbb{R}_{T}\right)}=0 \text {. }
$$

Now, a careful reading of the proof of [29, chapter III, Theorem 3.3] shows that the solution of the problem (3.26) in the space $W_{2}^{0,1}\left(\mathbb{R}_{T}\right)$ is unique and satisfies

$$
\|\tilde{\Delta}\|_{W_{2}^{0,1}\left(\mathbb{R}_{T}\right)} \leq C_{9}\left(\int_{0}^{T}\left(\int_{\mathbb{R}}\left|\tilde{\psi}_{k}\right|^{q_{1}} \mathrm{~d} x\right)^{q_{2} / q_{1}} \mathrm{~d} t\right)^{1 / q_{2}}
$$

for all $q_{1} \in[2, \infty], q_{2} \in[2,4]$ with $\frac{1}{q_{1}}+\frac{2}{q_{2}}=1$, provided that

$$
\int_{0}^{T}\left(\int_{\mathbb{R}}\left|A^{\prime}(R) R_{x}\right|^{q_{1}} \mathrm{~d} x\right)^{q_{2} / q_{1}} \mathrm{~d} t<\infty .
$$

We choose $q_{1}=q_{2}=3$, so that the latter condition is satisfied. In addition, by (3.27)

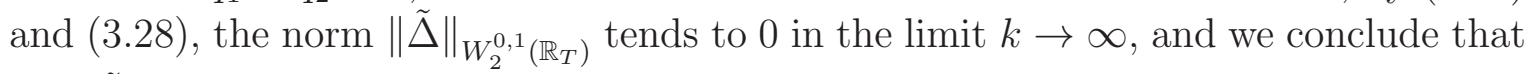
$U=\tilde{U} \in W_{q}^{1,2}\left(\mathbb{R}_{T}\right)$, as claimed.

\section{Proof of Proposition 2.2 (C)}

The proof of Prop. 2.2 (C) consists of four steps. In Step 1 we convert the variational formula $I_{\iota, \mu}(\gamma)<\infty$ into the formula (4.6) corresponding to a suitable one-dimensional reversible diffusion. Step 2 then deduces the existence of a sufficiently regular solution to the corresponding backward Cauchy problem (4.7), which enables us to employ Dirichlet form calculus for establishing in Step 3 the integrability of $R_{x x}^{2} / R_{x}$ for $R=R^{(\gamma)}$. From the latter we deduce in Step 4 that $R_{t}^{2} / R_{x}$ is integrable.

Step 1. The functions $a(t, x)=A(R)$ and $b(R)$ are uniformly bounded and our assumption that $I_{\iota, \mu}(\gamma)<\infty$ implies that $\mathrm{d} \gamma \mathrm{d} t$ has density $R_{x} \in L^{3}\left(\mathbb{R}_{T}\right)$ with respect to Lebesgue measure $\mathrm{d} m$ (by Prop. 2.2(A)). These facts imply by multiple applications 
16 AMIR DEMBO, MYKHAYLO SHKOLNIKOV, S. R. SRINIVASA VARADHAN, AND OFER ZEITOUNI

of Hölder's inequality that the functional $g \mapsto \Phi_{\gamma}(g)-(g, g)_{\gamma}$ in (2.4) is continuous with respect to the norm

$$
\|g\|_{\infty}+\left\|g_{t}\right\|_{L^{3 / 2}\left(\mathbb{R}_{T}\right)}+\left\|g_{x}\right\|_{L^{3 / 2}\left(\mathbb{R}_{T}\right)}+\left\|g_{x}\right\|_{L^{3}\left(\mathbb{R}_{T}\right)}+\left\|g_{x x}\right\|_{L^{3 / 2}\left(\mathbb{R}_{T}\right)} .
$$

Therefore, denoting by $\widetilde{W}_{3 / 2}^{1,2}\left(\mathbb{R}_{T}\right)$ the sub-space of all $g \in C_{b}\left(\mathbb{R}_{T}\right)$ for which $g_{t}, g_{x x} \in$ $L^{3 / 2}\left(\mathbb{R}_{T}\right)$ and $g_{x} \in L^{3 / 2}\left(\mathbb{R}_{T}\right) \cap L^{3}\left(\mathbb{R}_{T}\right)$, the assumption $I_{\iota, \mu}(\gamma)<\infty$ amounts to

$$
\sup _{g \in \widetilde{W}_{3 / 2}^{1,2}\left(\mathbb{R}_{T}\right)}\left[(\gamma, g)(T)-(\gamma, g)(0)-\int_{0}^{T}\left(\gamma, \mathcal{R}^{\gamma} g+a g_{x}^{2}\right)(t) \mathrm{d} t\right]<\infty
$$

for $\mathcal{R}^{\gamma}$ of (2.1). Now, fixing $\psi \in C^{\infty}(\mathbb{R})$ such that $\lim _{|x| \rightarrow \infty} \frac{\psi(x)}{|x|}=1,\left\|\psi^{\prime}\right\|_{\infty}<\infty$ and $\alpha_{0}:=e^{-\psi(x)} \mathrm{d} x$ is a probability measure, we introduce the parabolic operator

$$
\mathcal{R}^{\gamma, \psi}=\frac{\partial}{\partial t}+e^{\psi(x)} \frac{\partial}{\partial x} a(t, x) e^{-\psi(x)} \frac{\partial}{\partial x}=\mathcal{R}^{\gamma}+\left(a_{x}-a \psi^{\prime}-b(R)\right) \frac{\partial}{\partial x}
$$

and show next that (4.1) implies that

$$
\sup _{g \in \widetilde{W}_{3 / 2}^{1,2}\left(\mathbb{R}_{T}\right)}\left[(\gamma, g)(T)-\log \left(\int_{\mathbb{R}} e^{g(0, x)} \mathrm{d} \alpha_{0}\right)-\int_{0}^{T}\left(\gamma, \mathcal{R}^{\gamma, \psi} g+a g_{x}^{2}\right)(t) \mathrm{d} t\right]<\infty .
$$

Indeed, by Cauchy-Schwarz we have the bound

$$
\kappa_{3}:=\int_{\mathbb{R}_{T}}\left(b(R)-a_{x}+a \psi^{\prime}\right) g_{x} R_{x} \mathrm{~d} m \leq C_{2} \sqrt{(g, g)_{\gamma}}
$$

for the finite, positive

$$
C_{2}:=\underline{a}^{-1 / 2}\left(\|b\|_{\infty} T^{1 / 2}+\left\|A^{\prime}\right\|_{\infty}\left\|R_{x}\right\|_{L^{3}\left(\mathbb{R}_{T}\right)}^{3 / 2}\right)+\left\|\sqrt{a} \psi^{\prime}\right\|_{\infty} T^{1 / 2} .
$$

Further, the implication

$$
\begin{aligned}
& \forall \lambda>0: \lambda \kappa_{1} \leq C_{1}+\lambda^{2} \kappa_{2}, \quad \kappa_{3} \leq C_{2} \sqrt{\kappa_{2}} \\
\Longrightarrow & \forall \tilde{\lambda}>0: \tilde{\lambda} \kappa_{1}+\tilde{\lambda} \kappa_{3} \leq 2 C_{1}+\frac{1}{2} C_{2}^{2}+\tilde{\lambda}^{2} \kappa_{2}
\end{aligned}
$$

holds for all $\kappa_{1}, \kappa_{3} \in \mathbb{R}$ and $\kappa_{2}, C_{1}, C_{2}$ positive. Therefore, scaling the test functions $g$ in (4.1) and (4.3) by $\lambda>0$ and $\tilde{\lambda}=2 \lambda$, respectively, then considering (4.4) for $C_{1}=I_{\iota, \mu}(\gamma), \kappa_{1}=\Phi_{\gamma}(g)$ and $\kappa_{2}=(g, g)_{\gamma}$ proves that the change from $\mathcal{R}^{\gamma}$ to $\mathcal{R}^{\gamma, \psi}$ in (4.1) does not make the supremum infinite. Moreover, the change in value due to the terms corresponding to the initial condition, is bounded by

$$
\sup _{g \in \widetilde{W}_{3 / 2}^{1,2}\left(\mathbb{R}_{T}\right)}\left[\int_{\mathbb{R}} g(0, x) \mathrm{d} \mu-\log \int_{\mathbb{R}} e^{g(0, x)} \mathrm{d} \alpha_{0}\right] \leq H\left(\mu \mid \alpha_{0}\right)
$$

(see for example [10, Lemma 6.2.13]). The latter relative entropy is finite since

$$
H\left(\mu \mid \alpha_{0}\right)=\int_{\mathbb{R}} \log \frac{\mathrm{d} \mu}{\mathrm{d} \alpha_{0}} \mathrm{~d} \mu=\int_{\mathbb{R}} \log \frac{\theta}{e^{-\psi}} \mathrm{d} \mu \leq \int_{\mathbb{R}}|\psi| \mathrm{d} \mu+\int_{\mathbb{R}} \log \theta \mathrm{d} \mu,
$$

while $\int_{\mathbb{R}}|x| \mathrm{d} \mu<\infty$ and $\theta \in L^{q_{0}}(\mathbb{R})$ for some $q_{0}>1$ (by definition, for $\mu \in M_{1}^{(0)}(\mathbb{R})$ ).

Next, let $\mathcal{E} \widetilde{W}_{3 / 2}^{1,2}\left(\mathbb{R}_{T}\right)$ denote the collection of $u: \mathbb{R}_{T} \mapsto(0, \infty)$ such that $\log u=g \in$ $\widetilde{W}_{3 / 2}^{1,2}\left(\mathbb{R}_{T}\right)$. It is easy to verify that $\mathcal{E} \widetilde{W}_{3 / 2}^{1,2}\left(\mathbb{R}_{T}\right)$ consists of all positive $u \in \widetilde{W}_{3 / 2}^{1,2}\left(\mathbb{R}_{T}\right)$ 
which are bounded away from zero, in terms of which (4.3) becomes

$$
\sup _{u \in \mathcal{E} \widetilde{W}_{3 / 2}^{1,2}\left(\mathbb{R}_{T}\right)}\left[(\gamma, \log u)(T)-\log \int_{\mathbb{R}} u(0, x) \mathrm{d} \alpha_{0}-\int_{0}^{T}\left(\gamma, \frac{\mathcal{R}^{\gamma, \psi} u}{u}\right)(t) \mathrm{d} t\right]<\infty .
$$

Step 2. We claim that to every $f \in \overline{\mathcal{S}}$ corresponds $u \in \mathcal{E} \widetilde{W}_{3 / 2}^{1,2}\left(\mathbb{R}_{T}\right)$ such that

$$
\mathcal{R}^{\gamma, \psi} u-f u=0, \quad u(T, \cdot)=1
$$

where all terms of (4.7) are then in $L^{3 / 2}\left(\mathbb{R}_{T}\right)$ (by definition $u_{x}, R_{x} \in L^{r}\left(\mathbb{R}_{T}\right)$ for all $r \in[3 / 2,3]$, hence $\left(a_{x}-a \psi^{\prime}\right) u_{x} \in L^{3 / 2}\left(\mathbb{R}_{T}\right)$ by the Cauchy-Schwarz inequality and boundedness of $A^{\prime}, A$ and $\psi^{\prime}$ ). Clearly, having such solution for (4.7) amounts to finding a solution $w$ of

$$
\mathcal{R}^{\gamma, \psi} w-f w=f, \quad w(T, \cdot)=0
$$

or, equivalently (see (4.2)), a solution of

$$
w_{t}+\left(a w_{x}\right)_{x}-\psi^{\prime} a w_{x}-f w=f, \quad w(T, \cdot)=0
$$

such that $u=(w+1) \in \mathcal{E} \widetilde{W}_{3 / 2}^{1,2}\left(\mathbb{R}_{T}\right)$. To this end, we employ [27, Theorem 6.2] together with the method of continuity to first get a generalized solution $w$ of (4.8) in the space $W_{6}^{0,1}\left(\mathbb{R}_{T}\right) \cap W_{2}^{0,1}\left(\mathbb{R}_{T}\right)$. Indeed, the norm bound in [27, inequality (6.3)] extends to a norm bound for functions in $\mathcal{H}_{6}^{1} \cap \mathcal{H}_{2}^{1}$ with respect to the norms $\|\cdot\|_{\mathbb{H}_{6}^{-1}}+\|\cdot\|_{\mathbb{H}_{2}^{-1}}$ and $\|\cdot\|_{\mathbb{H}_{6}^{1}}+\|\cdot\|_{\mathbb{H}_{2}^{1}}$ defined in [27] (one only needs to add the norm bounds [27, inequality (6.3)] for $p=6$ and $p=2$ ). Applying the method of continuity (see [30, section III.2]), and relying on such a refined norm estimate to interpolate between the PDE (4.8) and the corresponding PDE with a smooth coefficient $a$, we find a solution of (4.8) in $\mathcal{H}_{6}^{1} \cap \mathcal{H}_{2}^{1}$, which in particular belongs to $W_{6}^{0,1}\left(\mathbb{R}_{T}\right) \cap W_{2}^{0,1}\left(\mathbb{R}_{T}\right)$. Moreover, by Hölder's inequality $a_{x} w_{x} \in L^{2}\left(\mathbb{R}_{T}\right) \cap L^{3 / 2}\left(\mathbb{R}_{T}\right)$ (since $a_{x}=A^{\prime}(R) R_{x} \in L^{3}\left(\mathbb{R}_{T}\right)$ and $w_{x} \in L^{r}\left(\mathbb{R}_{T}\right.$ ) for all $r \in[2,6])$. Hence, refining the norm bounds in [27, Theorem 4.1] to an estimate on the norms in $L^{2}\left(\mathbb{R}_{T}\right) \cap L^{3 / 2}\left(\mathbb{R}_{T}\right)$ and applying the method of continuity in a similar fashion, we also have a generalized solution $\hat{w} \in W_{2}^{1,2}\left(\mathbb{R}_{T}\right) \cap W_{3 / 2}^{1,2}\left(\mathbb{R}_{T}\right)$ of the equation

$$
\hat{w}_{t}+a \hat{w}_{x x}-a \psi^{\prime} \hat{w}_{x}-f \hat{w}=-a_{x} w_{x}+f, \quad \hat{w}(T, \cdot)=0 .
$$

Proceeding to show that $w=\hat{w}$, we let $\left\{\phi_{k}, k \in \mathbb{N}\right\}$ be the same truncation sequence as in the proof of Prop. 2.2 (B), fix $k \in \mathbb{N}$, and set $\Delta:=\phi_{k}(\hat{w}-w)$. Then, $\Delta \in W_{2}^{0,1}\left(\mathbb{R}_{T}\right)$ is a generalized solution of

$$
\Delta_{t}+\left(a \Delta_{x}\right)_{x}-\left(a_{x}+\psi^{\prime} a\right) \Delta_{x}-f \Delta=\psi_{k}, \quad \Delta(T, \cdot)=0,
$$

where

$$
\psi_{k}=\phi_{k}^{\prime \prime} a(\hat{w}-w)+2 \phi_{k}^{\prime} a(\hat{w}-w)_{x}-\phi_{k}^{\prime} \psi^{\prime} a(\hat{w}-w) .
$$

As in the derivation of (3.27), since $\max \left(\left|\phi_{k}^{\prime}\right|,\left|\phi_{k}^{\prime \prime}\right|\right) \leq 2 \cdot \mathbf{1}_{|x| \in[k, k+1]}$, having $a, \psi^{\prime}$ uniformly bounded and $(\hat{w}-w),(\hat{w}-w)_{x} \in L^{2}\left(\mathbb{R}_{T}\right)$ implies that

$$
\lim _{k \rightarrow \infty}\left\|\psi_{k}\right\|_{L^{2}\left(\mathbb{R}_{T}\right)}=0 .
$$

Hence, writing $\Delta=\phi_{k} \hat{w}-\phi_{k} w$, following the paragraph after the statement of [29, chapter III, Theorem 3.3], and applying the energy inequality of [29, chapter III, Theorem 2.1], with $r=q=2$ and $n=1$ (so that $\frac{2}{r}+\frac{n}{q} \leq \frac{n+4}{2}$ ), we conclude that

$$
\|\Delta\|_{L^{2}\left(\mathbb{R}_{T}\right)} \leq C_{3}\left(\int_{0}^{T}\left(\int_{\mathbb{R}}\left|\psi_{k}\right|^{q} \mathrm{~d} x\right)^{r / q} \mathrm{~d} t\right)^{1 / r} \rightarrow 0, \quad \text { as } \quad k \rightarrow \infty .
$$


Therefore, $m$-a.e. $w=\hat{w}$ on $\mathbb{R}_{T}$. All in all, we have found a solution $u$ to (4.7) such that $w=u-1$ is an element of $W_{2}^{1,2}\left(\mathbb{R}_{T}\right) \cap W_{3 / 2}^{1,2}\left(\mathbb{R}_{T}\right)$, and hence also $u_{x}=w_{x} \in L^{6}\left(\mathbb{R}_{T}\right)$ (by the parabolic Sobolev inequality in the form of [29, chapter II, Lemma 3.3] for $p=6$ and $q=2$ ).

It thus remains only to show that $u \in C_{b}\left(\mathbb{R}_{T}\right)$ and that $u$ is bounded away from 0 on $\mathbb{R}_{T}$. To establish this we first apply [29, chapter III, Theorem 5.2] to find a generalized solution $\tilde{w}$ of (4.8) in the subspace of $W_{2}^{0,1}\left(\mathbb{R}_{T}\right)$ whose elements satisfy

$$
\operatorname{esssup}_{t \in[0, T]} \int_{\mathbb{R}} \tilde{w}(t, x)^{2} \mathrm{~d} x+\int_{\mathbb{R}_{T}} \tilde{w}_{x}^{2} \mathrm{~d} m<\infty .
$$

Next, we apply [1, Theorem 10 (vi)], with the constant $\gamma>0$ there being arbitrarily small, to conclude that $\tilde{u}:=\tilde{w}+1$ has to be the unique generalized solution of (4.7) in the sense of [1]. It is thus given by

$$
\tilde{u}(t, x)=\int_{\mathbb{R}} \Gamma(t, x ; T, y) \mathrm{d} y
$$

with $\Gamma$ denoting the weak fundamental solution of (4.7), defined as in [1, Section 6]. Now, 1, Theorem C] implies that $\tilde{u}$ is locally Hölder continuous in $(t, x)$, and hence continuous in $(t, x)$ on $[0, T) \times \mathbb{R}$. Putting this together with [1, Theorem 10 (vi)], we conclude that $\tilde{u}$ is continuous on the whole of $\mathbb{R}_{T}$. Finally, we use the heat kernel estimates on $\Gamma$ from [1, Theorem 7] to conclude that $\tilde{u}$ has to be bounded between two positive constants. Therefore, all we need to show now is that $\tilde{u}=u$ or, equivalently, $\tilde{w}=w$. To this end, re-using the truncation sequence $\left\{\phi_{k}\right\}$, we fix $k \in \mathbb{N}$ and set $\tilde{\Delta}:=\phi_{k}(\tilde{w}-w)$. Then $\tilde{\Delta} \in W_{2}^{0,1}\left(\mathbb{R}_{T}\right)$ is a generalized solution of

$$
\tilde{\Delta}_{t}+\left(a \tilde{\Delta}_{x}\right)_{x}-\psi^{\prime} a \tilde{\Delta}_{x}-f \tilde{\Delta}=\tilde{\psi}_{k}, \quad \tilde{\Delta}(T, \cdot)=0
$$

where

$$
\tilde{\psi}_{k}=\phi_{k}^{\prime \prime} a(\tilde{w}-w)+2 \phi_{k}^{\prime} a(\tilde{w}-w)_{x}+\phi_{k}^{\prime} a_{x}(\tilde{w}-w)-\phi_{k}^{\prime} \psi^{\prime} a(\tilde{w}-w) .
$$

The $L^{2}\left(\mathbb{R}_{T}\right)$-norm of $\phi_{k}^{\prime} a_{x}(\tilde{w}-w)$ decays as $k \rightarrow \infty$ (because $a_{x} \in L^{3}\left(\mathbb{R}_{T}\right)$ and $\|\tilde{w}-w\|_{L^{6}\left(S_{k, 4} \cap S_{-k, 4}\right)}$ is uniformly bounded in $\left.k\right)$. Thus, with $w, \tilde{w}, w_{x}, \tilde{w}_{x} \in L^{2}\left(\mathbb{R}_{T}\right)$ we deduce as in the derivation of (4.10) that

$$
\lim _{k \rightarrow \infty}\left\|\tilde{\psi}_{k}\right\|_{L^{2}\left(\mathbb{R}_{T}\right)}=0 .
$$

This implies, as in the derivation of (4.11), that $\|\tilde{\Delta}\|_{L^{2}\left(\mathbb{R}_{T}\right)} \rightarrow 0$ as $k \rightarrow \infty$, and consequently that $m$-a.e. $w=\tilde{w}$ on $\mathbb{R}_{T}$.

Step 3. In view of (4.7), we get from (4.6) that

$$
\infty>\sup _{f \in \overline{\mathcal{S}}}\left[-\log \int_{\mathbb{R}} u^{(f)}(0, x) \mathrm{d} \alpha_{0}-\int_{0}^{T}(\gamma, f)(t) \mathrm{d} t\right],
$$

where $u^{(f)} \in \mathcal{E} \widetilde{W}_{3 / 2}^{1,2}\left(\mathbb{R}_{T}\right)$ satisfies (4.7). We then deduce that, for some $C \in(0, \infty)$,

$$
\infty>\sup _{g \in \overline{\mathcal{S}}}\left[-\int_{0}^{T}\left(\gamma, g_{x}+C g^{2}\right)(t) \mathrm{d} t\right]=\sup _{g \in \overline{\mathcal{S}}} \int_{\mathbb{R}_{T}}\left(g R_{x x}-C g^{2} R_{x}\right) \mathrm{d} m
$$

by showing that the $L^{2}\left(\alpha_{0}\right)$-norm of $u^{(f)}(0, \cdot)$ (and hence also $\left.\log \int_{\mathbb{R}} u^{(f)}(0, x) \mathrm{d} \alpha_{0}\right)$, is uniformly bounded over all $f=g_{x}+C g^{2}$ with $g \in \overline{\mathcal{S}}$. To this end, recall from (4.2) 
that $\mathcal{R}^{\gamma, \psi} v=v_{t}+\mathcal{L} v$ for $v \in \widetilde{W}_{3 / 2}^{1,2}\left(\mathbb{R}_{T}\right)$ and the bounded linear operator

$$
\mathcal{L} v=e^{\psi}\left(a e^{-\psi} v_{x}\right)_{x}: \widetilde{W}_{3 / 2}^{1,2}\left(\mathbb{R}_{T}\right) \mapsto L^{3 / 2}\left(\mathbb{R}_{T}\right) .
$$

We further set $\mathcal{L}_{t} \phi=e^{\psi}\left(a(t, x) e^{-\psi} \phi^{\prime}\right)_{x}$ for $\phi \in C_{c}^{\infty}(\mathbb{R})$. Then, considering positive $\phi_{k} \in \overline{\mathcal{S}}$ that converge to $u^{(f)}$ in $\widetilde{W}_{3 / 2}^{1,2}\left(\mathbb{R}_{T}\right)$, we deduce from (4.7) that, for any $s \in[0, T]$,

$$
\begin{aligned}
1-\left\|u^{(f)}(s, \cdot)\right\|_{L^{2}\left(\alpha_{0}\right)}^{2} & =2 \int_{s}^{T} \mathrm{~d} t \int_{\mathbb{R}} u^{(f)} u_{t}^{(f)} \mathrm{d} \alpha_{0} \\
& =-2 \int_{s}^{T} \mathrm{~d} t \int_{\mathbb{R}} u^{(f)}\left(\mathcal{L} u^{(f)}-f u^{(f)}\right) \mathrm{d} \alpha_{0} \\
& \geq-2 \int_{s}^{T} \lambda_{f}(t)\left\|u^{(f)}(t, \cdot)\right\|_{L^{2}\left(\alpha_{0}\right)}^{2} \mathrm{~d} t
\end{aligned}
$$

where

$$
\lambda_{f}(t)=\sup _{\phi \in C_{c}^{\infty}(\mathbb{R}): \phi \geq 0,\|\phi\|_{L^{2}\left(\alpha_{0}\right)}=1} \int_{\mathbb{R}} \phi\left(\mathcal{L}_{t} \phi-f(t, \cdot) \phi\right) \mathrm{d} \alpha_{0} .
$$

Recall that $\mathrm{d} \alpha_{0}=e^{-\psi} \mathrm{d} x$, so integrating by parts and then taking $\phi \mapsto \sqrt{\phi}$ yields

$$
\begin{aligned}
\lambda_{f}(t) & =\sup _{\phi \in C_{c}^{\infty}(\mathbb{R}): \phi \geq 0,\|\phi\|_{L^{2}\left(\alpha_{0}\right)}=1}\left[-\int_{\mathbb{R}} a(t, \cdot)\left(\phi^{\prime}\right)^{2} \mathrm{~d} \alpha_{0}-\int_{\mathbb{R}} f(t, \cdot) \phi^{2} \mathrm{~d} \alpha_{0}\right] \\
& =\sup _{\phi \in C_{c}^{\infty}(\mathbb{R}): \phi \geq 0,\|\phi\|_{L^{1}\left(\alpha_{0}\right)}=1}\left[-\frac{1}{4} \int_{\mathbb{R}} \frac{\left(\phi^{\prime}\right)^{2}}{\phi} a(t, \cdot) \mathrm{d} \alpha_{0}-\int_{\mathbb{R}} f(t, \cdot) \phi \mathrm{d} \alpha_{0}\right] .
\end{aligned}
$$

Further, for any $g \in \overline{\mathcal{S}}$ and smooth $\phi$ such that $\phi \alpha_{0} \in M_{1}(\mathbb{R})$, we get by integration by parts and the Cauchy-Schwarz inequality that

$$
\left|\int_{\mathbb{R}} g_{x}(t, \cdot) \phi \mathrm{d} \alpha_{0}\right|=\left|\int_{\mathbb{R}} g(t, \cdot)\left(\phi^{\prime}-\phi \psi^{\prime}\right) \mathrm{d} \alpha_{0}\right| \leq \sqrt{v_{1} v_{2}}+\left\|\psi^{\prime}\right\|_{\infty} \sqrt{v_{2}},
$$

with $v_{1}=\int_{\mathbb{R}} \frac{\left(\phi^{\prime}\right)^{2}}{\phi} \mathrm{d} \alpha_{0}$ and $v_{2}=v_{2}(t)=\int_{\mathbb{R}} g^{2}(t, \cdot) \phi \mathrm{d} \alpha_{0}$. Hence, for $f=g_{x}+C g^{2}$ with $g \in \overline{\mathcal{S}}$, we end up with

$$
\sup _{t \in[0, T]}\left\{\lambda_{f}(t)\right\} \leq \sup _{v_{1}, v_{2} \geq 0}\left[-\frac{1}{4} \underline{a} v_{1}+\sqrt{v_{1} v_{2}}+\left\|\psi^{\prime}\right\|_{\infty} \sqrt{v_{2}}-C v_{2}\right] .
$$

For $C>0$ large enough, the right side of (4.17) is finite, hence applying Grönwall's lemma for $s \mapsto\left\|u^{(f)}(T-s, \cdot)\right\|_{L^{2}\left(\alpha_{0}\right)}^{2}$ we deduce from (4.15) that $\left\|u^{(f)}(0, \cdot)\right\|_{L^{2}\left(\alpha_{0}\right)}$ is bounded uniformly (over such $f$ ), as claimed.

Next, let $\widehat{\mathbb{H}}$ denote the closure of $\overline{\mathcal{S}}$ with respect to the $L^{2}\left(R_{x} \mathrm{~d} m\right)$-norm. From (4.14) we know that, for some finite $C_{1}, C$ and all $g \in \overline{\mathcal{S}}$,

$$
\left|\int_{\mathbb{R}_{T}} g R_{x x} \mathrm{~d} m\right| \leq C_{1}+C\|g\|_{\mathbb{H}}^{2} .
$$

Hence, $g \mapsto \int_{\mathbb{R}_{T}} g R_{x x} \mathrm{~d} m$ extends to a bounded linear functional on $\widehat{\mathbb{H}}$, which by the Riesz representation theorem is of the form $g \mapsto(g, \hat{h})_{\widehat{\mathbb{H}}}$ for some $\hat{h} \in \widehat{\mathbb{H}}$. The identity $\int_{\mathbb{R}_{T}} g R_{x x} \mathrm{~d} m=\int_{\mathbb{R}_{T}} g \hat{h} R_{x} \mathrm{~d} m$ for all $g \in \overline{\mathcal{S}}$ implies that $m$-a.e. if $R_{x}=0$ then $R_{x x}=0$, with $\hat{h}=\frac{R_{x x}}{R_{x}}$ (subject to our running convention that $\frac{0}{0}=0$ ). Consequently,

$$
\int_{\mathbb{R}_{T}} \frac{R_{x x}^{2}}{R_{x}} \mathrm{~d} m=\int_{\mathbb{R}_{T}} \hat{h}^{2} R_{x} \mathrm{~d} m=\|\hat{h}\|_{\mathbb{\mathbb { H }}}^{2}<\infty .
$$


Step 4. Following the derivation of (4.18) out of (4.14), if

$$
\sup _{g \in \overline{\mathcal{S}}} \int_{\mathbb{R}_{T}}\left(2 g R_{t}-g^{2} R_{x}\right) \mathrm{d} m<\infty
$$

then $\int_{\mathbb{R}_{T}} \frac{R_{t}^{2}}{R_{x}} \mathrm{~d} m<\infty$. Further, plugging in (4.19) the value of $R_{t}$ from the PDE (3.12), all the terms of which are in $L^{3 / 2}\left(\mathbb{R}_{T}\right)$, we find that (4.19) amounts to

$$
\sup _{g \in \overline{\mathcal{S}}} \int_{\mathbb{R}_{T}}\left(2 g\left[A^{\prime}(R) R_{x}+\hat{h} A(R)+h A(R)\right]-g^{2}\right) R_{x} \mathrm{~d} m<\infty,
$$

for $\hat{h} \in \widehat{\mathbb{H}}$ of (4.18) and $h \in L^{2}\left(R_{x} \mathrm{~d} m\right)$ of (2.8). Point-wise optimizing in (4.20) over the value of $g(t, x)$ at each point of $\mathbb{R}_{T}$, bounds the supremum by the finite $\left\|A^{\prime}(R) R_{x}+\hat{h} A(R)+h A(R)\right\|_{L^{2}\left(R_{x} \mathrm{~d} m\right)}^{2}$, thereby completing the proof.

Remark 4.1. A crucial step in the proof of Prop. 2.2 (C) consists of showing that the continuous $u=w+1$ solving (4.7), with $w \in W_{2}^{1,2}\left(\mathbb{R}_{T}\right) \cap W_{3 / 2}^{1,2}\left(\mathbb{R}_{T}\right)$, is further bounded and bounded away from 0 . In doing so we relied on the results of [1, but we note in passing that with some additional work such positivity can be obtained from [28, Corollary 4.6], bypassing the need for [1].

\section{Proof of Proposition 2.5}

Fixing throughout $\iota \in(0,1]$ and $\mu \in M_{1}^{(\iota)}(\mathbb{R})$, we start with the convexity of the functionals from which $J_{\iota, \mu}(\cdot)$ is composed, followed by its use in establishing convergence results for $\widetilde{J}(\cdot)$.

Lemma 5.1. The functionals

$$
J^{(1)}(R)=\int_{\mathbb{R}_{T}} \frac{R_{t}^{2}}{R_{x}} \mathrm{~d} m, \quad J^{(2)}(R)=\int_{\mathbb{R}_{T}} \frac{R_{x x}^{2}}{R_{x}} \mathrm{~d} m, \quad J^{(3)}(R)=\int_{\mathbb{R}_{T}} R_{x}^{3} \mathrm{~d} m
$$

are convex on the set $\mathcal{F}=\mathcal{F}_{3 / 2}$ of (1.7).

Proof. The convexity of $J^{(3)}(\cdot)$ is an immediate consequence of the convexity of $x \mapsto x^{3}$ on $[0, \infty)$. Further, recall from Steps 3 and 4 in the proof of Prop. 2.2 (C), that on $\mathcal{F}$

$$
\begin{aligned}
& J^{(1)}(R)=\sup _{g \in \overline{\mathcal{S}}} \int_{\mathbb{R}_{T}}\left(2 g R_{t}-g^{2} R_{x}\right) \mathrm{d} m, \\
& J^{(2)}(R)=\sup _{g \in \overline{\mathcal{S}}} \int_{\mathbb{R}_{T}}\left(2 g R_{x x}-g^{2} R_{x}\right) \mathrm{d} m,
\end{aligned}
$$

so each of these functionals, being a supremum of linear functionals, must therefore be convex.

Lemma 5.2. Suppose $A(\cdot) \geq \underline{a}>0$ with $b(\cdot)$ and $A^{\prime}(\cdot)$ continuous and bounded. Let $R=R^{(\gamma)}$ for $\gamma \in \mathcal{C}$ such that $\bar{J}_{\iota, \mu}(\gamma)<\infty$ and suppose the strictly positive probability densities $R_{x}^{\epsilon} \in C^{1,1}\left(\mathbb{R}_{T}\right)$ are such that

$$
\limsup _{\epsilon \downarrow 0} J^{(\ell)}\left(R^{\epsilon}\right) \leq J^{(\ell)}(R), \quad \ell=1,2,3,
$$

$R^{\epsilon} \rightarrow R$ uniformly on compacts, $R_{x}^{\epsilon} \rightarrow R_{x}$ in $L^{p}\left(\mathbb{R}_{T}\right), p \in[2,3]$ and $m$-a.e. $R_{t}^{\epsilon} \rightarrow R_{t}$, $R_{x x}^{\epsilon} \rightarrow R_{x x}$. If $\gamma^{\epsilon}=R_{x}^{\epsilon} \mathrm{d} x$ are such that $\gamma^{\epsilon}(0) \in M_{1}^{(0)}(\mathbb{R})$, then

$$
\lim _{\epsilon \downarrow 0} \widetilde{J}\left(\gamma^{\epsilon}\right)=J_{\iota, \mu}(\gamma)
$$


Proof. Step 1. We first show that as $\epsilon \downarrow 0$ :

$$
\begin{gathered}
\int_{\mathbb{R}_{T}}\left|\frac{R_{t}^{\epsilon}}{\left(R_{x}^{\epsilon}\right)^{1 / 2}}-\frac{R_{t}}{\left(R_{x}\right)^{1 / 2}}\right|^{2} \mathrm{~d} m \rightarrow 0, \\
\int_{\mathbb{R}_{T}}\left|\frac{R_{x x}^{\epsilon}}{\left(R_{x}^{\epsilon}\right)^{1 / 2}}-\frac{R_{x x}}{\left(R_{x}\right)^{1 / 2}}\right|^{2} \mathrm{~d} m \rightarrow 0, \\
\int_{\mathbb{R}_{T}}\left|\left(R_{x}^{\epsilon}\right)^{3 / 2}-\left(R_{x}\right)^{3 / 2}\right|^{2} \mathrm{~d} m \rightarrow 0 .
\end{gathered}
$$

To this end, with $\left|x_{1}-x_{2}\right|^{2} \leq\left|x_{1}^{2}-x_{2}^{2}\right|$ whenever $x_{1}, x_{2} \in \mathbb{R}_{+}$and $R_{x}^{\epsilon} \rightarrow R_{x}$ in $L^{2}\left(\mathbb{R}_{T}\right)$, it follows that, for $\epsilon \downarrow 0$,

$$
\int_{\mathbb{R}_{T}}\left|\left(R_{x}^{\epsilon}\right)^{1 / 2}-\left(R_{x}\right)^{1 / 2}\right|^{2} \mathrm{~d} m \rightarrow 0 .
$$

Similarly, combining the inequality $\left|x_{1}^{3}-x_{2}^{3}\right| \leq \frac{3}{2}\left|x_{1}^{2}-x_{2}^{2}\right| \max \left(x_{1}, x_{2}\right)$ (which holds for all $x_{1}, x_{2} \in \mathbb{R}_{+}$) with Hölder's inequality we find that

$$
\begin{aligned}
\int_{\mathbb{R}_{T}}\left|\left(R_{x}^{\epsilon}\right)^{3 / 2}-\left(R_{x}\right)^{3 / 2}\right|^{2} \mathrm{~d} m & \leq \frac{9}{4} \int_{\mathbb{R}_{T}}\left|R_{x}^{\epsilon}-R_{x}\right|^{2} \max \left(R_{x}^{\epsilon}, R_{x}\right) \mathrm{d} m \\
\leq & \frac{9}{4}\left(\int_{\mathbb{R}_{T}}\left|R_{x}^{\epsilon}-R_{x}\right|^{3} \mathrm{~d} m\right)^{2 / 3}\left(\int_{\mathbb{R}_{T}} \max \left(R_{x}^{\epsilon}, R_{x}\right)^{3} \mathrm{~d} m\right)^{1 / 3} .
\end{aligned}
$$

By assumption $R_{x}^{\epsilon} \rightarrow R_{x}$ in $L^{3}\left(\mathbb{R}_{T}\right)$ and, due to (5.2) for $\ell=3$, the norms $\left\|R_{x}^{\epsilon}\right\|_{L^{3}\left(\mathbb{R}_{T}\right)}$ are uniformly bounded, thereby yielding (5.6).

Turning to prove (5.4), we let $c^{\epsilon}=R_{t}^{\epsilon}\left(R_{x}^{\epsilon}\right)^{-1 / 2}$ which by (5.2) with $\ell=1$ is $L^{2}\left(\mathbb{R}_{T}\right)$ bounded. Hence, for any sequence $\epsilon_{k} \downarrow 0$ we have that $c^{\epsilon} \rightarrow c^{*}$ weakly in $L^{2}\left(\mathbb{R}_{T}\right)$ along some sub-sequence (by the Banach-Alaoglu theorem, where $c^{*} \in L^{2}\left(\mathbb{R}_{T}\right)$ may depend on the chosen sub-sequence). By the triangle inequality, we thus get from (5.7) that along this sub-sequence, for any fixed $\psi \in C_{c}\left(\mathbb{R}_{T}\right)$,

$$
\int_{\mathbb{R}_{T}}\left(R_{x}^{\epsilon}\right)^{1 / 2} c^{\epsilon} \psi \mathrm{d} m \rightarrow \int_{\mathbb{R}_{T}}\left(R_{x}\right)^{1 / 2} c^{*} \psi \mathrm{d} m .
$$

Recall that $R_{t} \in L^{3 / 2}\left(\mathbb{R}_{T}\right)$ (since $R \in \mathcal{F}$ ), and by assumption $m$-a.e. $\left(R_{x}^{\epsilon}\right)^{1 / 2} c^{\epsilon}=R_{t}^{\epsilon} \rightarrow$ $R_{t}$. Thus, for any fixed $\psi \in C_{c}\left(\mathbb{R}_{T}\right)$, the 1.h.s. of (5.8) converges to $\int_{\mathbb{R}_{T}} R_{t} \psi \mathrm{d} m$ as $\epsilon \downarrow 0$, resulting with

$$
\int_{\mathbb{R}_{T}}\left(R_{x}\right)^{1 / 2} c^{*} \psi \mathrm{d} m=\int_{\mathbb{R}_{T}} R_{t} \psi \mathrm{d} m .
$$

Further, with $\left(R_{x}\right)^{1 / 2} \in L^{6}\left(\mathbb{R}_{T}\right)$ and $c^{*} \in L^{2}\left(\mathbb{R}_{T}\right)$, by Hölder's inequality $\left(R_{x}\right)^{1 / 2} c^{*} \in$ $L^{3 / 2}\left(\mathbb{R}_{T}\right)$, so from (5.9) we conclude that $m$-a.e. $c^{*}=R_{t}\left(R_{x}\right)^{-1 / 2}$, independently of the sequence $\epsilon_{k}$. That is, $R_{t}^{\epsilon}\left(R_{x}^{\epsilon}\right)^{-1 / 2} \rightarrow R_{t}\left(R_{x}\right)^{-1 / 2}$ weakly in $L^{2}\left(\mathbb{R}_{T}\right)$ when $\epsilon \downarrow 0$. Together with the $L^{2}\left(\mathbb{R}_{T}\right)$-norm-bound of (5.2) for $\ell=1$, this yields the (strong) convergence of (5.4).

Finally, the same argument, just with $R_{t}^{\epsilon}$ replaced by $R_{x x}^{\epsilon}$, yields (5.5).

Step 2. To deduce (5.3) from (5.4) -(5.7), recall that $J_{\iota, \mu}(\gamma)<\infty$ requires $\gamma \in \mathcal{A}_{\iota, \mu}$ and, in particular, that $R=R^{(\gamma)} \in C_{b}\left(\mathbb{R}_{T}\right)$ satisfies (3.1) for some $M=M_{\alpha}$ finite (and all $\alpha>0)$. Thus, our assumption that $R^{\epsilon} \rightarrow R$ uniformly on compact sets, combined with the monotonicity of the distribution functions $x \mapsto R^{\epsilon}(t, x), x \mapsto R(t, x)$ and 
(3.1), yields that $R^{\epsilon} \rightarrow R$ uniformly on $\mathbb{R}_{T}$, when $\epsilon \downarrow 0$. This, and the assumed continuity of $A^{\prime}$ and $b$ on $[0,1]$, show that as $\epsilon \downarrow 0$,

$$
\sigma\left(R^{\epsilon}\right) \rightarrow \sigma(R), \quad A^{\prime}\left(R^{\epsilon}\right) \rightarrow A^{\prime}(R), \quad b\left(R^{\epsilon}\right) \rightarrow b(R) \text { uniformly on } \mathbb{R}_{T} .
$$

Moreover, all functions appearing in (5.10) are uniformly bounded on $\mathbb{R}_{T}$. Putting this together with the uniform positivity of $\sigma$ and (5.4)-(5.7), we have the following convergences in $L^{2}\left(\mathbb{R}_{T}\right)$ when $\epsilon \downarrow 0$,

$$
\begin{gathered}
\frac{1}{\sigma\left(R^{\epsilon}\right)} \frac{R_{t}^{\epsilon}}{\left(R_{x}^{\epsilon}\right)^{1 / 2}} \rightarrow \frac{1}{\sigma(R)} \frac{R_{t}}{\left(R_{x}\right)^{1 / 2}}, \\
\sigma\left(R^{\epsilon}\right) \frac{R_{x x}^{\epsilon}}{\left(R_{x}^{\epsilon}\right)^{1 / 2}} \rightarrow \sigma(R) \frac{R_{x x}}{\left(R_{x}\right)^{1 / 2}} \\
\frac{A^{\prime}\left(R^{\epsilon}\right)}{\sigma\left(R^{\epsilon}\right)}\left(R_{x}^{\epsilon}\right)^{3 / 2} \rightarrow \frac{A^{\prime}(R)}{\sigma(R)}\left(R_{x}\right)^{3 / 2} \\
\frac{b\left(R^{\epsilon}\right)}{\sigma\left(R^{\epsilon}\right)}\left(R_{x}^{\epsilon}\right)^{1 / 2} \rightarrow \frac{b(R)}{\sigma(R)}\left(R_{x}\right)^{1 / 2}
\end{gathered}
$$

By Hölder's inequality the finiteness of $J^{(\ell)}\left(R^{\epsilon}\right), \ell=1,2,3$ implies that $R^{\epsilon}=R^{\left(\gamma^{\epsilon}\right)} \in \mathcal{F}$, and by our assumptions, also $\gamma^{\epsilon} \in \widetilde{\mathcal{A}}$. Thus, as $\epsilon \downarrow 0$ we have that

$$
\begin{aligned}
\widetilde{J}\left(\gamma^{\epsilon}\right) & =\frac{1}{2}\left\|\frac{R_{t}^{\epsilon}-\left(A\left(R^{\epsilon}\right) R_{x}^{\epsilon}\right)_{x}}{\sigma\left(R^{\epsilon}\right)\left(R_{x}^{\epsilon}\right)^{1 / 2}}+\frac{b\left(R^{\epsilon}\right)}{\sigma\left(R^{\epsilon}\right)}\left(R_{x}^{\epsilon}\right)^{1 / 2}\right\|_{L^{2}\left(\mathbb{R}_{T}\right)}^{2} \\
& \longrightarrow \frac{1}{2}\left\|\frac{R_{t}-\left(A(R) R_{x}\right)_{x}}{\sigma(R)\left(R_{x}\right)^{1 / 2}}+\frac{b(R)}{\sigma(R)}\left(R_{x}\right)^{1 / 2}\right\|_{L^{2}\left(\mathbb{R}_{T}\right)}^{2}=J_{\iota, \mu}(\gamma) .
\end{aligned}
$$

This completes the proof of Lemma 5.2.

Under Asmp. 1.2(a),(b) and (d), our key result provides the conclusions of Prop. 2.5 for $J_{\iota, \mu}(\gamma)<\infty$ and some $\gamma^{\epsilon}$ in the collection $\mathcal{G}_{\iota}^{\star}$ that we define next (c.f. Definition 2.4 of $\mathcal{G})$.

Definition 5.3. Let $\mathcal{G}_{\iota}^{\star}$ be the subset of $\left\{\gamma \in \mathcal{C}: J_{\iota, \mu}(\gamma)<\infty\right.$, some $\left.\mu \in M_{1}^{(\iota)}(\mathbb{R})\right\}$ such that $R=R^{(\gamma)} \in C_{b}^{\infty}\left(\mathbb{R}_{T}\right)$ with $R_{x}$ strictly positive and

$$
\left|\partial_{t}^{j} \partial_{x}^{k} R(t, x)\right| \leq c_{j, k} R_{\star}(x) \quad \forall(t, x) \in \mathbb{R}_{T}
$$

for some $\left\{c_{j, k}\right.$, finite $\left.j, k \in \mathbb{N}\right\}$ and

$$
R_{\star}(x):=\sup _{t \in[0, T]}\{\widetilde{R}(t, x)\}, \quad \widetilde{R}(t, x):=1-R(t,|x|)+R(t,-|x|) .
$$

Proposition 5.4. Suppose $A \geq \underline{a}>0$ with $b$ and $A^{\prime}$ continuous and bounded. If $J_{\iota, \mu}(\gamma)<\infty$ for some $\mu \in M_{1}^{(\iota)}(\mathbb{R})$, then there exist $\left\{\gamma^{\epsilon}\right\} \subset \mathcal{G}_{\iota}^{\star}$ such that $\gamma^{\epsilon} \rightarrow \gamma$ in $\mathcal{C}$ as $\epsilon \downarrow 0$, $\sup _{\epsilon} \int|x|^{1+\iota} \mathrm{d} \gamma^{\epsilon}(0)$ is finite, and (5.3) holds.

Proof. The proof consists of three steps. In Step 1 we construct $\gamma^{\delta, \epsilon} \in \mathcal{C}$ whose smooth CDF paths $R^{\delta, \epsilon}=R^{\left(\gamma^{\delta, \epsilon}\right)} \in C_{b}^{\infty}\left(\mathbb{R}_{T}\right)$ satisfy (5.15) and having strictly positive PDF-s $R_{x}^{\delta, \epsilon}$. Step 2 confirms that $\gamma^{\delta, \epsilon} \rightarrow \gamma$ in $\mathcal{C}$ when $(\delta, \epsilon) \rightarrow(0,0)$ and that $\gamma^{\delta, \epsilon} \in \mathcal{A}_{\iota, \mu}$ for $\mu=\gamma^{\delta, \epsilon}(0)$ whose $(1+\iota)$-th moments are bounded, uniformly over $(\delta, \epsilon)$. Then, relying on Lemma 5.2, we verify in Step 3 that (5.3) holds for $\delta=\delta(\epsilon)$ small enough (so, in particular, such $\gamma^{\delta, \epsilon} \in \mathcal{G}_{\iota}^{\star}$ ).

Step 1. Let $\phi \in C^{\infty}(\mathbb{R})$ be a strictly positive probability density with $\int|x|^{k} \phi(x) \mathrm{d} x<$ $\infty$ and $\left|\phi^{(k)}(x)\right| \leq c_{k} \phi(x)$ for some finite $c_{k}, k \geq 1$ and all $x \in \mathbb{R}$ ( for example, the 
smoothing near $x=0$ of $e^{-2|x|}$ provides such $\phi$ ). With $\phi_{\epsilon}(y)=\epsilon^{-1} \phi(y / \epsilon)$, for each $\delta, \epsilon \in(0,1)$ we consider the function

$$
S^{\delta, \epsilon}(t, x)=\int_{\mathbb{R}} R\left((t-3 \delta)_{+}, y\right) \phi_{\epsilon}(x-y) \mathrm{d} y
$$

on $\mathbb{R}_{T+3 \delta}$. Then, fixing a probability density $\psi \in C_{c}^{\infty}(\mathbb{R})$ supported on $[0,3]$ with

$$
c_{\psi}=\inf _{s \in[1,2]}\{\psi(s)\}>0,
$$

we set $\psi_{\delta}(s)=\delta^{-1} \psi(s / \delta)$ and consider

$$
R^{\delta, \epsilon}(t, x)=\int_{0}^{T+3 \delta} S^{\delta, \epsilon}(s, x) \psi_{\delta}(s-t) \mathrm{d} s, \quad \delta, \epsilon \in(0,1), \quad(t, x) \in \mathbb{R}_{T} .
$$

With $\psi(t) \phi(x)$ a probability density on $\mathbb{R}_{T}$, since each $R(t, \cdot)$ is a CDF, so are $S^{\delta, \epsilon}(t, \cdot)$ and $R^{\delta, \epsilon}(t, \cdot)$. By the strict positivity of $\phi$ we have the same for $S_{x}^{\delta, \epsilon}$, and thereby also for $R_{x}^{\delta, \epsilon}$. Next, with $\phi(\cdot)$ smooth, $S^{\delta, \epsilon}(t, \cdot) \in C_{b}^{\infty}(\mathbb{R})$, for each $t, \epsilon$ and $\delta$, hence $R^{\delta, \epsilon} \in$ $C_{b}^{\infty}\left(\mathbb{R}_{T}\right)$ by smoothness of $\psi$. Moreover, as $\phi(\cdot)$ point-wise controls its derivatives it follows that, for each $k \geq 1$ and all $(t, x) \in \mathbb{R}_{T}$,

$$
\left|\partial_{x}^{k} S^{\delta, \epsilon}(t, x)\right| \leq c_{k} \epsilon^{-k} S^{\delta, \epsilon}(t, x) .
$$

Further, with $\int \phi_{\epsilon}(x-y) \mathrm{d} y=1$, the same bound holds for $1-S^{\delta, \epsilon}$, resulting with

$$
\left|\partial_{x}^{k} S^{\delta, \epsilon}(t, x)\right| \leq c_{k} \epsilon^{-k}\left[\left(1-S^{\delta, \epsilon}(t, x)\right) \wedge S^{\delta, \epsilon}(t, x)\right] \leq c_{k} \epsilon^{-k} \widetilde{S}^{\delta, \epsilon}(t, x)
$$

(where $\widetilde{S}^{\delta, \epsilon}$ is related to $S^{\delta, \epsilon}$ analogously to (5.16) ). Thus, having $\psi(\cdot)$ smooth yields, by (5.20) and (5.19), that

$$
\left|\partial_{t}^{j} \partial_{x}^{k} R^{\delta, \epsilon}(t, x)\right| \leq\left\|\psi_{\delta}^{(j)}\right\|_{\infty} c_{k} \epsilon^{-k} V^{\delta, \epsilon}(t, x)
$$

for all $j, k \in \mathbb{N}$ where

$$
V^{\delta, \epsilon}(t, x)=\int_{0}^{3 \delta} \widetilde{S}^{\delta, \epsilon}(t+u, x) \mathrm{d} u .
$$

Clearly, for any $\delta>0$ and $(t, x) \in \mathbb{R}_{T}$,

$$
V^{\delta, \epsilon}(t, x) \leq \frac{3 \delta}{c_{\psi}} \sup _{t \in[0, T]}\left\{\int_{0}^{3 \delta} \widetilde{S}^{\delta, \epsilon}(t+u, x) \psi_{\delta}(u) \mathrm{d} u\right\}=\frac{3 \delta}{c_{\psi}} R_{\star}^{\delta, \epsilon}(x),
$$

for $R_{\star}(\cdot)$ of (5.16), which together with (5.21) implies that $R^{\delta, \epsilon}$ satisfies (5.15).

Step 2. Since $S^{\delta, \epsilon}(t, x)=S^{\delta, \epsilon}(0, x)$ when $t \in[0,3 \delta]$, upon specializing (5.19) to $t=0$, we get the smooth CDF-S

$$
R^{\delta, \epsilon}(0, x)=\mathbb{E}\left[R\left(0, x-Y_{\epsilon}\right)\right]=: \Theta_{\epsilon}(x),
$$

for $Y_{\epsilon}$ of density $\phi_{\epsilon}$. Our assumption that $\gamma(0)=\mu \in M_{1}^{(\iota)}(\mathbb{R})$ translates to $\gamma^{\delta, \epsilon}(0) \in$ $M_{1}^{(\iota)}(\mathbb{R})$ with uniformly bounded $(1+\iota)$-moments (since $Y_{\epsilon}$ has this property and $f \mapsto\left(\int f(x)^{q_{0}} \mathrm{~d} x, \int|x|^{1+\iota} f(x) \mathrm{d} x\right)$ is convex over probability densities). Similarly, from (5.17) and (5.19)

$$
\int_{\mathbb{R}_{T}}|x|^{1+\iota} \mathrm{d} \gamma^{\delta, \epsilon}(t) \mathrm{d} t \leq \int_{\mathbb{R}_{T}} \mathbb{E}\left[\left|x-Y_{\epsilon}\right|^{1+\iota}\right] \mathrm{d} \gamma(t) \mathrm{d} t+3 \delta \int_{\mathbb{R}} \mathbb{E}\left[\left|x-Y_{\epsilon}\right|^{1+\iota}\right] \mathrm{d} \gamma(0),
$$

with the r.h.s. finite, since $\gamma$ satisfies (1.6),$\gamma(0) \in M_{1}^{(\iota)}(\mathbb{R})$, and $Y_{\epsilon}$ has finite moments of all order. That is, $\gamma^{\delta, \epsilon}$ satisfy the moment condition (1.6) and are thus in $\mathcal{A}_{\iota, \gamma} \gamma^{\delta, \epsilon}(0)$. In addition, by dominated convergence, $R^{\delta, \epsilon} \rightarrow R$ uniformly on compacts, so in particular, $\gamma^{\delta, \epsilon} \rightarrow \gamma$ in $\mathcal{C}$. 
Step 3. By construction, $m$-a.e. $R_{x}^{\delta, \epsilon} \rightarrow R_{x}, R_{t}^{\delta, \epsilon} \rightarrow R_{t}$ and $R_{x x}^{\delta, \epsilon} \rightarrow R_{x x}$, whenever $(\delta, \epsilon) \rightarrow(0,0)$. Thus, from Lemma 5.2 we get (5.3) for $\delta=\delta(\epsilon)$ small, once we show that

$$
\limsup _{\epsilon \downarrow 0} \limsup _{\delta \downarrow 0} J^{(\ell)}\left(R^{\delta, \epsilon}\right) \leq J^{(\ell)}(R), \quad \ell=1,2,3
$$

(indeed, by Egorov's theorem, (5.23) for $\ell=3$ implies that $R_{x}^{\delta, \epsilon} \rightarrow R_{x}$ in $L^{p}\left(\mathbb{R}_{T}\right)$, $p \in[2,3]$ ). Turning to prove (5.23), recall from its definition that $J_{\iota, \mu}(\gamma)<\infty$ implies $R=R^{(\gamma)} \in \mathcal{F}$ and

$$
J^{(\ell)}(R)=\int_{0}^{3 \delta} \psi_{\delta}(s) \mathrm{d} s \int_{\mathbb{R}} J^{(\ell)}(R(\cdot, y+\cdot)) \phi_{\epsilon}(y) \mathrm{d} y<\infty \quad \ell=1,2,3 .
$$

Hence, starting with the functional $J^{(1)}(\cdot)$, we get from the definition of $R^{\delta, \epsilon}$, upon applying Lemma 5.1 twice, that

$$
J^{(1)}\left(R^{\delta, \epsilon}\right) \leq \int_{0}^{3 \delta} J^{(1)}\left(S^{\delta, \epsilon}(s+\cdot, \cdot)\right) \psi_{\delta}(s) \mathrm{d} s \leq J^{(1)}(R),
$$

hence (5.23) holds for $\ell=1$. Next, consider the functionals

$$
\widehat{J}^{(2)}(F)=\int_{\mathbb{R}} \frac{F^{\prime \prime}(x)^{2}}{F^{\prime}(x)} \mathrm{d} x, \quad \widehat{J}^{(3)}(F)=\int_{\mathbb{R}} F^{\prime}(x)^{3} \mathrm{~d} x,
$$

which, as in the proof of Lemma 5.1, are convex on the set of twice differentiable CDF-s. Thus, for $\ell=2,3$, we get by the same argument as before that

$$
J^{(\ell)}\left(R^{\delta, \epsilon}\right) \leq J^{(\ell)}(R)+3 \delta \widehat{J}^{(\ell)}\left(\Theta_{\epsilon}\right) .
$$

Our choice of $\phi_{\epsilon}$ results with $\left|\Theta_{\epsilon}^{\prime \prime}\right| \leq c_{1} \epsilon^{-1} \Theta_{\epsilon}^{\prime}$ and uniformly bounded PDF $\Theta_{\epsilon}^{\prime}$. Consequently, $\widehat{J}^{(\ell)}\left(\Theta_{\epsilon}\right)<\infty$ and taking $\delta \downarrow 0$ establishes (5.23) for $\ell=2,3$.

Specializing to $\iota=1$ and applying Prop. 5.4 to approximate the given $\gamma \in \mathcal{C}$ of finite $J_{1, \mu}(\gamma)$ by a suitable sequence from $\mathcal{G}_{1}^{\star}$, the proof of Prop. 2.5 is thus completed by considering our next lemma (to get $\gamma^{\ell} \in \mathcal{G}$ that suitably converge to any given $\left.\gamma \in \mathcal{G}_{1}^{\star}\right)$.

Lemma 5.5. Suppose $\gamma \in \mathcal{G}_{\iota}^{\star}$ and Asmp. 1.2(a),(b) and (d) hold. Then, there exist $\mu \in M_{1}^{(\iota-1)}(\mathbb{R})$ such that $\gamma^{\ell}=\left(1-\ell^{-1}\right) \gamma+\ell^{-1} \mu \in \mathcal{G}$ with $\widetilde{J}\left(\gamma^{\ell}\right) \rightarrow \widetilde{J}(\gamma)$, and $\gamma^{\ell} \rightarrow \gamma$ in $\mathcal{C}$.

Proof. Recall $c_{0,1}=c_{0,1}(\gamma)$ from (5.15). We begin by showing that, with $\eta=$ $1 /\left(12 c_{0,1}\right)$, the function $R_{\star}$ of (5.16) is such that

$$
|x-y| \leq 3 \eta \quad \Longrightarrow \quad R_{\star}(y) \leq 2 R_{\star}(x) .
$$

Indeed, since $R_{\star}(z)=R_{\star}(-z)$ is continuous and non-increasing in $|z|$, it suffices to prove that $R_{\star}(y) \leq 2 R_{\star}(x)$ for $|x| \in[|y|,|y|+3 \eta]$ and $x y \geq 0$. To this end, note that by (5.15), for all $t \in[0, T]$,

$$
|R(t, y)-R(t, x)|=\left|\int_{x}^{y} R_{x}(t, z) \mathrm{d} z\right| \leq c_{0,1}\left|\int_{x}^{y} R_{\star}(z) \mathrm{d} z\right| \leq \frac{1}{4} R_{\star}(y) .
$$

The preceding implies that, for $\widetilde{R}(t, x)$ of (5.16),

$$
\widetilde{R}(t, y) \leq \widetilde{R}(t, x)+\frac{1}{2} R_{\star}(y),
$$

so taking the maximum over $t$ results with $R_{\star}(y) \leq R_{\star}(x)+\frac{1}{2} R_{\star}(y)$, as claimed. 
We next claim that

$$
\int_{\mathbb{R}}|x|^{\iota} R_{\star}(x) \mathrm{d} x<\infty .
$$

Indeed, taking $\widehat{\eta}=1 /\left(8 c_{1,0}\right)>0$, we have that, for $|t-s| \leq 2 \widehat{\eta}$,

$$
|R(t, x)-R(s, x)| \leq c_{1,0}|t-s| R_{\star}(x) \leq \frac{1}{4} R_{\star}(x),
$$

out of which we deduce that $\widetilde{R}(s, x) \leq \widetilde{R}(t, x)+\frac{1}{2} R_{\star}(x)$. Considering $s \in[0, T]$ such that $\widetilde{R}(s, x)=R_{\star}(x)$, we thus find that $\widetilde{R}(t, x) \geq \frac{1}{2} R_{\star}(x)$ throughout a sub-interval of length at least $2 \widehat{\eta}$. Consequently, for all $x \geq 0$,

$$
\widehat{\eta} R_{\star}(x) \leq \int_{0}^{T} \widetilde{R}(t, x) \mathrm{d} t
$$

hence by Fubini's theorem,

$$
\frac{\iota+1}{2} \widehat{\eta} \int_{\mathbb{R}}|x|^{\iota} R_{\star}(x) \mathrm{d} x \leq(\iota+1) \int_{0}^{\infty} x^{\iota} \mathrm{d} x \int_{0}^{T} \widetilde{R}(t, x) \mathrm{d} t=\int_{\mathbb{R}_{T}}|x|^{\iota+1} \mathrm{~d} \gamma(t) \mathrm{d} t
$$

which for $\gamma \in \mathcal{G}_{\iota}^{\star}$ is finite in view of (1.6).

Continuing with the proof of the lemma, let $\kappa=\int_{\mathbb{R}} R_{\star}(y) \mathrm{d} y$, which by (5.26) is finite. Then, for $\eta>0$ of (5.25) and $\psi \in C_{c}^{\infty}(\mathbb{R})$ supported on $[0,3]$ as in Prop. 5.4, we construct the PDF

$$
r^{\prime}(x):=\frac{1}{\kappa} \int_{\mathbb{R}} R_{\star}(y) \psi_{\eta}(x-y) \mathrm{d} y,
$$

and, for each $\epsilon>0$, consider the path $\gamma^{\epsilon} \in \mathcal{C}$ associated with the CDF-s

$$
R^{\epsilon}(t, x):=(1-\epsilon) R(t, x)+\epsilon r(x) .
$$

Since $R_{x}$ is strictly positive, so are $R_{x}^{\epsilon}$. Further $r \in C_{b}^{\infty}(\mathbb{R})$, and consequently also $R^{\epsilon} \in C_{b}^{\infty}\left(\mathbb{R}_{T}\right)$. For $\epsilon \downarrow 0$ we clearly have that $R^{\epsilon} \rightarrow R$ uniformly on compacts (so $\gamma^{\epsilon} \rightarrow \gamma$ in $\mathcal{C}$ ), and $m$-a.e. $R_{t}^{\epsilon} \rightarrow R_{t}, R_{x x}^{\epsilon} \rightarrow R_{x x}$. Since $r^{\prime}(\cdot)$ and $R_{x}(t, \cdot)$ are both uniformly bounded PDF-s, obviously also $R_{x}^{\epsilon} \rightarrow R_{x}$ in $L^{p}\left(\mathbb{R}_{T}\right)$ for all $p \in[2,3]$. Thus, in view of Lemmas 5.1 and 5.2 , we have that $\widetilde{J}\left(\gamma^{\epsilon}\right) \rightarrow \widetilde{J}(\gamma)$ provided we show that $\widehat{J}^{(\ell)}(r), \ell=2,3$ of $(5.24)$ are finite. The finiteness of $\widehat{J}^{(3)}(r)$ is trivial, for $r^{\prime}$ is a bounded PDF, whereas $\widehat{J}^{(2)}(r)<\infty$ due to the boundedness of $\left|r^{\prime \prime}(x)\right| / r^{\prime}(x)$. To see the latter, note that for $c_{\psi}>0$ as in (5.18), by (5.25) and (5.27),

$$
\frac{c_{\psi}}{2 \eta} R_{\star}(x) \leq \frac{c_{\psi}}{\eta} \inf _{y \in[x-3 \eta, x]}\left\{R_{\star}(y)\right\} \leq \kappa r^{\prime}(x) \leq \sup _{y \in[x-3 \eta, x]}\left\{R_{\star}(y)\right\} \leq 2 R_{\star}(x) .
$$

Similarly, $\kappa\left|r^{\prime \prime}(x)\right| \leq 2\left\|\psi^{\prime}\right\|_{1} \eta^{-1} R_{\star}(x)$ which together with the l.h.s. of (5.28) implies that $\left|r^{\prime \prime}(x)\right| / r^{\prime}(x)$ is uniformly bounded (by $4\left\|\psi^{\prime}\right\|_{1} / c_{\psi}$ ).

Next, combining the r.h.s. of (5.28) with (5.26), we deduce that the $\iota$-th moment of $\mu=r^{\prime}(x) \mathrm{d} x$ is finite. It thus remains only to show that the continuous function

$$
h\left(R^{\epsilon}\right)=\frac{R_{t}^{\epsilon}-\left(A\left(R^{\epsilon}\right) R_{x}^{\epsilon}\right)_{x}}{A\left(R^{\epsilon}\right) R_{x}^{\epsilon}}
$$

is further uniformly bounded and globally Lipschitz in $x$. With $A$ bounded below, $A^{\prime}$, $R_{x}^{\epsilon}$ bounded above, and $R_{t}^{\epsilon}=(1-\epsilon) R_{t}$, the boundedness of $h\left(R^{\epsilon}\right)$ follows from that of $\left(\left|R_{t}\right|+\left|R_{x x}\right|+\left|r^{\prime \prime}\right|\right) / r^{\prime}$. To this end, we have just shown the uniform boundedness of $\left|r^{\prime \prime}(x)\right| / r^{\prime}(x)$ and recall from (5.15) that $\left|R_{t}(t, x)\right|+\left|R_{x x}(t, x)\right| \leq\left(c_{1,0}+c_{0,2}\right) R_{\star}(x)$, 
which by the l.h.s. of (5.28) is further bounded by $C r^{\prime}(x)$, for some $C=C(\gamma)$ finite. As for showing that $h\left(R^{\epsilon}\right)$ is globally Lipschitz continuous in $x$, note that

$$
\left[h\left(R^{\epsilon}\right)\right]_{x}=\frac{(1-\epsilon) R_{t x}-\left(A\left(R^{\epsilon}\right) R_{x}^{\epsilon}\right)_{x x}-h\left(R^{\epsilon}\right)\left(A\left(R^{\epsilon}\right) R_{x}^{\epsilon}\right)_{x}}{A\left(R^{\epsilon}\right) R_{x}^{\epsilon}} .
$$

Recall that $A(\cdot)$ is bounded below, $h\left(R^{\epsilon}\right), R_{x}, r^{\prime}$ and $\left(\left|R_{x x}\right|+\left|r^{\prime \prime}\right|\right) / r^{\prime}$ are bounded above, and $A^{\prime}$ is globally Lipschitz. We thus have that $\left|\left[h\left(R^{\epsilon}\right)\right]_{x}\right|$ is uniformly bounded, provided that $\left(\left|R_{t x}\right|+\left|R_{x x x}\right|+\left|r^{\prime \prime \prime}\right|\right) / r^{\prime}$ is uniformly bounded. The latter holds by the l.h.s. of (5.28), since from (5.20) we have that $\left|R_{t x}\right|+\left|R_{x x x}\right| \leq C R_{\star}$ for some $C=C(\gamma)$ finite, and by the same reasoning as above, we also get that $\kappa\left|r^{\prime \prime \prime}\right| \leq 2\left\|\psi^{\prime \prime}\right\|_{1} \eta^{-2} R_{\star}$.

\section{Proof of Proposition 2.6}

We suppose throughout this section that parts (a)-(c) of Asmp. 1.2 hold for some $\iota_{\star} \in(0,1]$ and use the simplified notations $I(\cdot)$ and $\mathcal{A}$ for $I_{\iota_{\star}, \rho_{0}}(\cdot)$ of (2.4) and $\mathcal{A}_{\iota_{\star}, \rho_{0}}$ of (1.6), respectively. In this setting we establish a local large deviations upper bound for $\rho^{N}$ being near $\gamma$, starting with $\gamma \in \mathcal{A}$ (where $I(\gamma)=\sup _{g \in \overline{\mathcal{S}}}\left[\Phi_{\gamma}(g)-(g, g)_{\gamma}\right]$ ).

Proposition 6.1. For each $\gamma \in \mathcal{A}$ and any $g \in \overline{\mathcal{S}}$ we have the upper bound

$$
\lim _{\delta \downarrow 0} \limsup _{N \rightarrow \infty} \frac{1}{N} \log \mathbb{P}\left(\rho^{N} \in B(\gamma, \delta)\right) \leq(g, g)_{\gamma}-\Phi_{\gamma}(g) .
$$

Proof. Fixing $g \in \overline{\mathcal{S}}$, for each $t \in[0, T]$ and $\xi \in \mathcal{C}$, we set

$$
H^{g}(\xi)(t)=\left(\xi, \mathcal{R}^{\xi} g\right)(t),
$$

with $\mathcal{R}^{\xi} g=g_{t}+b\left(R^{(\xi)}\right) g_{x}+A\left(R^{(\xi)}\right) g_{x x}$ of (2.1). Then, applying Itô's formula for the real-valued stochastic processes $Z_{N}^{g}(t):=\left(\rho^{N}, g\right)(t)$, one finds that

$$
Z_{N}^{g}(t)-Z_{N}^{g}(0)=\int_{0}^{t} H^{g}\left(\rho^{N}\right)(s) \mathrm{d} s+M_{N}^{g}(t),
$$

with the continuous martingale

$$
M_{N}^{g}(t):=\frac{1}{N} \sum_{i=1}^{N} \int_{0}^{t} \sigma\left(F_{\rho^{N}(s)}\left(X_{i}(s)\right)\right) g_{x}\left(s, X_{i}(s)\right) \mathrm{d} W_{i}(s) .
$$

Its quadratic variation is $\left\langle M_{N}^{g}\right\rangle(t)=\frac{1}{N} \int_{0}^{t} V^{g}\left(\rho^{N}\right)(s) \mathrm{d} s$, with

$$
V^{g}(\xi)(t):=2\left(\xi, A\left(R^{(\xi)}\right) g_{x}^{2}\right)(t) \text {. }
$$

Hence, by the martingale representation theorem (see [24, Theorem 3.4.2]),

$$
M_{N}^{g}(t)=\frac{1}{\sqrt{N}} \int_{0}^{t} \sqrt{V^{g}\left(\rho^{N}\right)(s)} \mathrm{d} \beta_{N}(s)
$$

for some one-dimensional standard Brownian motion $\beta_{N}$. Next, fixing $\gamma \in \mathcal{A}$, let

$$
\bar{H}^{g}(\gamma)(t)=\int_{0}^{t} H^{g}(\gamma)(s) \mathrm{d} s, \quad \bar{V}^{g}(\gamma)(t)=\int_{0}^{t} V^{g}(\gamma)(s) \mathrm{d} s
$$

and recall that, by (2.2) and (2.3),

$$
(g, g)_{\gamma}-\Phi_{\gamma}(g)=\frac{1}{2} \bar{V}^{g}(\gamma)(T)+(\gamma, g)(0)+\bar{H}^{g}(\gamma)(T)-(\gamma, g)(T) .
$$

We thus proceed to compare $Z_{N}^{g}(\cdot)$ with the process

$$
Y_{N}^{g}(t)=(\gamma, g)(0)+\bar{H}^{g}(\gamma)(t)+M_{N}^{g, \gamma}(t), \quad t \in[0, T],
$$


having the martingale part

$$
M_{N}^{g, \gamma}(t)=\frac{1}{\sqrt{N}} \int_{0}^{t} \sqrt{V^{g}(\gamma)(s)} \mathrm{d} \beta_{N}(s), \quad t \in[0, T] .
$$

Step 1. We first show that, on the event $\rho^{N} \in B(\gamma, \delta)$,

$$
\left\|Z_{N}^{g}-Y_{N}^{g}\right\|_{\infty}:=\sup _{t \in[0, T]}\left|Z_{N}^{g}(t)-Y_{N}^{g}(t)\right| \leq \epsilon,
$$

up to a probability which is negligible at our large deviations exponential scale (in the limit $N \rightarrow \infty$ followed by $\delta \downarrow 0)$. To this end, fixing $\rho \in B(\gamma, \delta)$ we note that

$$
\begin{aligned}
\int_{0}^{T}\left|H^{g}(\rho)(s)-H^{g}(\gamma)(s)\right| \mathrm{d} s \leq & \int_{0}^{T}\left|\left(\rho, \mathcal{R}^{\rho} g\right)(s)-\left(\rho, \mathcal{R}^{\gamma} g\right)(s)\right| \mathrm{d} s \\
& +\int_{0}^{T}\left|\left(\rho, \mathcal{R}^{\gamma} g\right)(s)-\left(\gamma, \mathcal{R}^{\gamma} g\right)(s)\right| \mathrm{d} s
\end{aligned}
$$

and setting $\widetilde{R}=R^{(\rho)}, R=R^{(\gamma)}$, we further have

$$
\begin{aligned}
\int_{0}^{T}\left|V^{g}(\rho)(s)-V^{g}(\gamma)(s)\right| \mathrm{d} s \leq & 2 \int_{0}^{T}\left|\left(\rho, A(\widetilde{R}) g_{x}^{2}\right)(s)-\left(\rho, A(R) g_{x}^{2}\right)(s)\right| \mathrm{d} s \\
& +2 \int_{0}^{T}\left|\left(\rho, A(R) g_{x}^{2}\right)(s)-\left(\gamma, A(R) g_{x}^{2}\right)(s)\right| \mathrm{d} s .
\end{aligned}
$$

Since $\gamma \in \mathcal{A}$, we know that $R \in C_{b}\left(\mathbb{R}_{T}\right)$ and consequently so are $\mathcal{R}^{\gamma} g$ and $A(R) g_{x}^{2}$, from which we deduce that the second term in both upper bounds tends to zero as $\delta \downarrow 0$, uniformly in $\rho \in B(\gamma, \delta)$. Now, recall that $b$ and $A$ are Lipschitz functions (under Asmp. 1.2(a)-(b)). Hence, to get the same uniform convergence for the first term in both upper bounds, it suffices to show that

$$
\lim _{\delta \downarrow 0} \sup _{\rho \in B(\gamma, \delta)} \int_{0}^{T}(\rho,|\widetilde{R}-R|)(s) \mathrm{d} s=0 .
$$

Moreover, fixing $\delta>0$, by definition of the metric $d(\cdot, \cdot)$ on $\mathcal{C}$, for any $\rho \in B(\gamma, \delta)$ and $(s, x) \in \mathbb{R}_{T}$ one has that

$$
R(s, x-\delta)-\delta \leq \widetilde{R}(s, x) \leq R(s, x+\delta)+\delta
$$

(see (1.5)), and hence, also

$$
|\widetilde{R}(s, x)-R(s, x)| \leq R(s, x+\delta)-R(s, x-\delta)+\delta .
$$

Recall that $R(s, x+\delta)-R(s, x-\delta)=\gamma(s,(x-\delta, x+\delta])$, resulting by Fubini's theorem and yet another application of the preceding bound with

$$
\begin{aligned}
(\rho,|\widetilde{R}-R|)(s) & \leq \delta+\int_{\mathbb{R}}(R(s, x+\delta)-R(s, x-\delta)) \rho(s, \mathrm{~d} x) \\
& =\delta+\int_{\mathbb{R}} \rho(s,[y-\delta, y+\delta)) \gamma(s, \mathrm{~d} y) \\
& \leq 3 \delta+\int_{\mathbb{R}}(R(s, y+3 \delta)-R(s, y-3 \delta)) \gamma(s, \mathrm{~d} y) .
\end{aligned}
$$

Integrating both sides over $s \in[0, T]$, we see that $(\underline{6.8})$ is a consequence of

$$
\lim _{\delta \downarrow 0} \int_{\mathbb{R}_{T}}(R(s, y+3 \delta)-R(s, y-3 \delta)) \gamma(s, \mathrm{~d} y) \mathrm{d} s=0,
$$


which in turn follows from the dominated convergence theorem and the fact that $R \in C_{b}\left(\mathbb{R}_{T}\right)$. All in all, we have shown that

$$
\begin{aligned}
& \lim _{\delta \downarrow 0} \sup _{\rho \in B(\gamma, \delta)} \int_{0}^{T}\left|H^{g}(\rho)(s)-H^{g}(\gamma)(s)\right| \mathrm{d} s=0, \\
& \lim _{\delta \downarrow 0} \sup _{\rho \in B(\gamma, \delta)} \int_{0}^{T}\left|V^{g}(\rho)(s)-V^{g}(\gamma)(s)\right| \mathrm{d} s=0 .
\end{aligned}
$$

Recall that $\left(\rho^{N}, g\right)(0) \rightarrow\left(\rho_{0}, g(0, \cdot)\right)=(\gamma, g)(0)$ by Asmp. 1.2(c) and the definition of $\mathcal{A}$. Hence, comparing (6.2) with (6.7), it immediately follows from (6.10) that, for any fixed $\epsilon>0$,

$$
\begin{aligned}
& \lim _{\delta \downarrow 0} \limsup _{N \rightarrow \infty} \frac{1}{N} \log \mathbb{P}\left(\rho^{N} \in B(\gamma, \delta),\left\|Z_{N}^{g}-Y_{N}^{g}\right\|_{\infty}>2 \epsilon\right) \\
& \quad \leq \lim _{\delta \downarrow 0} \limsup _{N \rightarrow \infty} \frac{1}{N} \log \mathbb{P}\left(\rho^{N} \in B(\gamma, \delta),\left\|M_{N}^{g}-M_{N}^{g, \gamma}\right\|_{\infty}>\epsilon\right) .
\end{aligned}
$$

Recall [32, Theorem 8.5.7] that $M_{N}^{g}(\cdot)-M_{N}^{g, \gamma}(\cdot)$ has the law of time-changed standard Brownian motion $\beta(\tau(\cdot))$, for $\tau(t)=\left\langle M_{N}^{g}-M_{N}^{g, \gamma}\right\rangle(t)$ (the quadratic variation process of the martingale $\left.M_{N}^{g}-M_{N}^{g, \gamma}\right)$. Moreover, on the event $\left\{\rho^{N} \in B(\gamma, \delta)\right\}$, we have that

$$
\tau(T)=\frac{1}{N} \int_{0}^{T}\left(\sqrt{V^{g}\left(\rho^{N}\right)(s)}-\sqrt{V^{g}(\gamma)(s)}\right)^{2} \mathrm{~d} s \leq \frac{1}{N} \kappa_{\gamma}(\delta),
$$

with $\kappa_{\gamma}(\delta) \rightarrow 0$ as $\delta \downarrow 0$ (due to the inequality $\left(\sqrt{x_{1}}-\sqrt{x_{2}}\right)^{2} \leq\left|x_{1}-x_{2}\right|$ for $x_{1}, x_{2} \geq 0$ and (6.11) $)$. Thus, from Bernstein's inequality for Brownian motion,

$$
\begin{aligned}
& \lim _{\delta \downarrow 0} \limsup _{N \rightarrow \infty} \frac{1}{N} \log \mathbb{P}\left(\rho^{N} \in B(\gamma, \delta),\left\|M_{N}^{g}-M_{N}^{g, \gamma}\right\|_{\infty}>\epsilon\right) \\
& \quad \leq \lim _{\delta \downarrow 0} \limsup _{N \rightarrow \infty} \frac{1}{N} \log \mathbb{P}\left(\sup _{t \in\left[0, \kappa_{\gamma}(\delta) / N\right]}|\beta(t)|>\epsilon\right) \leq-\lim _{\delta \downarrow 0}\left\{\frac{\epsilon^{2}}{2 \kappa_{\gamma}(\delta)}\right\}=-\infty .
\end{aligned}
$$

Step 2. Recall that, for any $\alpha_{1}, \alpha_{2} \in M_{1}(\mathbb{R})$,

$$
d_{B L}\left(\alpha_{1}, \alpha_{2}\right)=\sup _{\|f\|_{\infty}+\|f\|_{\text {Lip }} \leq 1}\left\{\left|\left(\alpha_{1}, f\right)-\left(\alpha_{2}, f\right)\right|\right\} \leq 2 d_{L}\left(\alpha_{1}, \alpha_{2}\right)
$$

(see [12, Corollary 11.6.5]). Hence, by (1.5) there exists $r_{g}:(0, \infty) \rightarrow(0, \infty)$ such that $\lim _{\delta \downarrow 0} r_{g}(\delta)=0$, and

$$
\rho \in B(\gamma, \delta) \quad \Longrightarrow \quad\|(\rho, g)-(\gamma, g)\|_{\infty} \leq r_{g}(\delta) .
$$

Recalling that $Z_{N}^{g}(t)=\left(\rho^{N}, g\right)(t)$, we thus have from Step 1 that, for any $\epsilon>0$,

$$
\begin{aligned}
& \lim _{\delta \downarrow 0} \limsup _{N \rightarrow \infty} \frac{1}{N} \log \mathbb{P}\left(\rho^{N} \in B(\gamma, \delta)\right) \\
& \quad=\lim _{\delta \downarrow 0} \limsup _{N \rightarrow \infty} \frac{1}{N} \log \mathbb{P}\left(\rho^{N} \in B(\gamma, \delta),\left\|Z_{N}^{g}-Y_{N}^{g}\right\|_{\infty} \leq \epsilon\right) \\
& \quad \leq \lim _{\delta \downarrow 0} \limsup _{N \rightarrow \infty} \frac{1}{N} \log \mathbb{P}\left(\left\|\left(\rho^{N}, g\right)-(\gamma, g)\right\|_{\infty} \leq r_{g}(\delta),\left\|Z_{N}^{g}-Y_{N}^{g}\right\|_{\infty} \leq \epsilon\right) \\
& \quad \leq \limsup _{N \rightarrow \infty} \frac{1}{N} \log \mathbb{P}\left(\left\|(\gamma, g)-Y_{N}^{g}\right\|_{\infty} \leq 2 \epsilon\right) .
\end{aligned}
$$


Therefore, it suffices to prove the simpler local large deviations upper bound

$$
\lim _{\epsilon \downarrow 0} \limsup _{N \rightarrow \infty} \frac{1}{N} \log \mathbb{P}\left(\left\|(\gamma, g)-Y_{N}^{g}\right\|_{\infty} \leq \epsilon\right) \leq-I^{g}((\gamma, g)),
$$

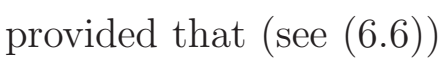

$$
I^{g}((\gamma, g)) \geq(\gamma, g)(T)-(\gamma, g)(0)-\bar{H}^{g}(\gamma)(T)-\frac{1}{2} \bar{V}^{g}(\gamma)(T) .
$$

Step 3. We establish (6.13) as a consequence of the LDP holding for $\left\{Y_{N}^{g}\right\}$ in the space $C([0, T], \mathbb{R})$ with a good rate function $I^{g}(\cdot)$. Indeed, note that by the timechange formalism for Itô integrals (see e.g. [32, Theorem 8.5.7]), the process $Y_{N}^{g}$ can be obtained as $\Psi\left(N^{-1 / 2} \tilde{\beta}\right)$ for a one-dimensional standard Brownian motion $\tilde{\beta}(t), t \geq 0$ and the deterministic operator

$$
\Psi: C([0, \infty), \mathbb{R}) \rightarrow C([0, T], \mathbb{R}), \quad(\Psi h)(t)=(\gamma, g)(0)+\bar{H}^{g}(\gamma)(t)+h\left(\bar{V}^{g}(\gamma)(t)\right) .
$$

Clearly, $\Psi$ is continuous with respect to uniform convergence on compacts in $C([0, \infty), \mathbb{R})$, hence by Schilder's theorem (see [10, Theorem 5.2.3]), and the contraction principle (see [10, Theorem 4.2.1]), the sequence $\left\{Y_{N}^{g}\right\}$ satisfies the LDP in $C([0, T], \mathbb{R})$ with the good rate function

$$
I^{g}(f)=\inf _{\{h: \Psi(h)=f\}} \frac{1}{2} \int_{0}^{S}\left(\frac{d h}{d u}\right)^{2} \mathrm{~d} u,
$$

where $S=\bar{V}^{g}(\gamma)(T)$ and the infimum is over all absolutely continuous functions $h$ on $[0, S]$, starting at $h(0)=0$, with Radon-Nikodym derivative $\frac{d h}{d u} \in L^{2}([0, S])$. In particular, since

$$
0 \leq \frac{1}{2} \int_{0}^{S}\left(\frac{d h}{d u}-1\right)^{2} \mathrm{~d} u=\frac{1}{2} \int_{0}^{S}\left(\frac{d h}{d u}\right)^{2} \mathrm{~d} u-h(S)+\frac{1}{2} S,
$$

it follows from the requirement $(\Psi h)(T)=(\gamma, g)(T)$ that

$$
I^{g}((\gamma, g)) \geq h(S)-\frac{1}{2} S=(\gamma, g)(T)-(\gamma, g)(0)-\bar{H}^{g}(\gamma)(T)-\frac{1}{2} S,
$$

which is precisely our claim (6.14).

We proceed with the local large deviations upper bound for paths $\gamma \notin \mathcal{A}$.

Proposition 6.2. If $\gamma \notin \mathcal{A}$, then

$$
\lim _{\delta \downarrow 0} \limsup _{N \rightarrow \infty} \frac{1}{N} \log \mathbb{P}\left(\rho^{N} \in B(\gamma, \delta)\right)=-\infty .
$$

Proof. Fixing $N$, let $\mathbb{Q}^{(b)}$ denote the law of the solution of the SDs $(1.2)$ with $\mathbb{Q}=\mathbb{Q}^{(0)}$ corresponding to the solution of (1.2) in case $b \equiv 0$. Recall that

$$
\frac{\mathrm{d} \mathbb{Q}^{(b)}}{\mathrm{d} \mathbb{Q}}=\exp \left(M_{N}^{b}(T)-\frac{1}{2}\left\langle M_{N}^{b}\right\rangle(T)\right)
$$

(see [24, Theorem 3.5.1]), with the continuous martingale

$$
M_{N}^{b}(t)=\sum_{i=1}^{N} \int_{0}^{t} \frac{b\left(F_{\rho^{N}(s)}\left(X_{i}(s)\right)\right)}{\sigma\left(F_{\rho^{N}(s)}\left(X_{i}(s)\right)\right)} \mathrm{d} W_{i}(s), \quad t \in[0, T],
$$


whose quadratic variation is $\left\langle M_{N}^{b}\right\rangle(t)=N \int_{0}^{t} U_{N}^{2}(s) \mathrm{d} s$, with $U_{N}(s)$ uniformly bounded by $\|b / \sigma\|_{\infty}$. Hence, setting $\kappa=\frac{T}{2}\|b / \sigma\|_{\infty}^{2}$, by the Cauchy-Schwarz inequality,

$$
\begin{aligned}
\mathbb{Q}^{(b)}\left(\rho^{N} \in B(\gamma, \delta)\right) & =\mathbb{Q}\left[e^{M_{N}^{b}(T)-\frac{1}{2}\left\langle M_{N}^{b}\right\rangle(T)} \mathbf{1}_{\left\{\rho^{N} \in B(\gamma, \delta)\right\}}\right] \\
& \leq e^{\kappa N} \mathbb{Q}\left[e^{2 M_{N}^{b}(T)-2\left\langle M_{N}^{b}\right\rangle(T)}\right]^{1 / 2} \mathbb{Q}\left(\rho^{N} \in B(\gamma, \delta)\right)^{1 / 2} \\
& =e^{\kappa N} \mathbb{Q}^{(2 b)}[1]^{1 / 2} \mathbb{Q}\left(\rho^{N} \in B(\gamma, \delta)\right)^{1 / 2} .
\end{aligned}
$$

Consequently, it suffices to establish (6.16) when $b \equiv 0$ in order to have the same conclusion for any other choice of $b(\cdot)$. With $\mathcal{A}$ independent of such choice, we proceed throughout the proof with $b \equiv 0$ (without loss of generality). In addition, since $\rho^{N} \rightarrow \rho_{0}$, the bound (6.16) trivially holds when $\gamma(0) \neq \rho_{0}$. Assuming hereafter that $\gamma(0)=\rho_{0}$, we distinguish the three reasons for $\gamma \notin \mathcal{A}$. We start with case (a) where $R^{(\gamma)} \notin C_{b}\left(\mathbb{R}_{T}\right)$, proceed to case (b) in which $R^{(\gamma)} \in C_{b}\left(\mathbb{R}_{T}\right)$ but $\gamma$ fails to satisfy the moment condition (1.6) for $\iota=\iota_{\star}$ of Asmp. 1.2(c), and finish with case (c), where $R^{(\gamma)} \in C_{b}\left(\mathbb{R}_{T}\right)$ and (1.6) holds, but $t \mapsto(\gamma, g)(t)$ is not absolutely continuous for some $g \in \overline{\mathcal{S}}$.

Case (a). By assumption $\gamma \in \mathcal{C}$ and $\gamma(0)$ has a density, so if $R^{(\gamma)} \notin C_{b}\left(\mathbb{R}_{T}\right)$ then necessarily $\gamma(s)(\{y\})=3 \epsilon$ for some $s \in(0, T], y \in \mathbb{R}$ and $\epsilon>0$. Fixing $N$ and $0<\delta<\epsilon$, it follows from (1.5), the definition of $d_{L}(\cdot, \cdot)$, and the union bound that, for $m=\lceil N \epsilon\rceil$,

$$
\begin{aligned}
\mathbb{P}\left(\rho^{N} \in B(\gamma, \delta)\right) & \leq \mathbb{P}\left(\rho^{N}(s)([y-\delta, y+\delta]) \geq \epsilon\right)=\mathbb{P}\left(\sum_{i=1}^{N} \mathbf{1}_{\left\{\left|X_{i}(s)-y\right| \leq \delta\right\}} \geq N \epsilon\right) \\
& \leq\left(\begin{array}{c}
N \\
m
\end{array}\right) \sup _{\left\{u_{j}, y_{j}\right\}} \mathbb{P}\left(\left|Z_{j}^{\left(u_{j}\right)}(s)-y_{j}\right| \leq \delta, \quad \forall j \leq m\right) .
\end{aligned}
$$

Here the supremum is over non-random $\left\{y_{1}, \ldots, y_{m}\right\}$ (into which we incorporated the initial conditions $\left.X_{i}(0)\right)$ and all [inf $\sigma$, sup $\sigma$ ]-valued processes $\left\{u_{1}, u_{2}, \ldots, u_{m}\right\}$ adapted to the filtration $\mathcal{H}_{t}$ generated by $\left\{W_{i}(r), r \in[0, t], 1 \leq i \leq N\right\}$, while

$$
Z_{j}^{\left(u_{j}\right)}(s)=\int_{0}^{s} u_{j}(r) \mathrm{d} W_{j}(r) .
$$

Each Itô integral in (6.20) is the $L^{2}$-limit of some $\mathcal{H}_{t}$-adapted stochastic integrals of processes which are piece-wise constant in time. Therefore, we can and shall assume hereafter that $\left\{u_{j}\right\}$ are simple processes, which are constant on each of the time intervals $\left[0, t_{1}\right), \ldots,\left[t_{k-1}, t_{k}\right)$ for some partition $0=t_{0}<t_{1}<\cdots<t_{k}=s$ and $k \in \mathbb{N}$ (possibly dependent on $N$ ). The probability we maximize in (6.19) is then

$$
\mathbb{E}\left[\prod_{j=1}^{m} G_{\delta}\left(\sum_{\ell=0}^{k-1} u_{j}\left(t_{\ell}\right) \Delta W_{j}\left(t_{\ell}\right), y_{j}\right)\right]
$$

for $\Delta W_{i}\left(t_{\ell}\right)=W_{i}\left(t_{\ell+1}\right)-W_{i}\left(t_{\ell}\right)$ and $G_{\delta}(z, v)=\mathbf{1}_{|z-v| \leq \delta}$. Such expectation, when conditioned upon

$$
\Gamma_{r}=\left\{u_{j}\left(t_{\ell}\right), \Delta W_{i}\left(t_{\ell}\right), \ell \leq k-2\right\} \cup\left\{u_{j}\left(t_{k-1}\right), \Delta W_{i}\left(t_{k-1}\right), i, j \neq r\right\}
$$

becomes

$$
\prod_{j \neq r} G_{\delta}\left(Z_{j}^{\left(u_{j}\right)}(s), y_{j}\right) \mathbb{E}\left[G_{\delta}\left(u_{r}\left(t_{k-1}\right) \Delta W_{r}\left(t_{k-1}\right)+Z_{r}^{\left(u_{r}\right)}\left(t_{k-1}\right), y_{r}\right) \mid \Gamma_{r}\right] .
$$


The value of $\Delta W_{r}\left(t_{k-1}\right)$ is independent of everything else, and clearly all that matters from $\Gamma_{r}$ to the choice of $u_{r}\left(t_{k-1}\right)$ that maximizes (6.21) is the value of $Z_{r}^{\left(u_{r}\right)}\left(t_{k-1}\right)$. We thus conclude that the optimal $u_{r}\left(t_{k-1}\right)$ may be assumed measurable with respect to the $\sigma$-algebra generated by $W_{r}(t), t \in\left[0, t_{k-1}\right]$ and $u_{r}\left(t_{\ell}\right), \ell \leq k-2$. Substituting optimal $u_{j}\left(t_{k-1}\right)$ of this type, for $1 \leq j \leq m$, changes the function $G_{\delta}$ considered in (6.21), but retains its form, namely we need thereafter to maximize

$$
\mathbb{E}\left[\prod_{j=1}^{m} G_{\delta}^{\prime}\left(Z_{j}^{\left(u_{j}\right)}\left(t_{k-1}\right), y_{j}\right)\right]
$$

for some (new) function $G_{\delta}^{\prime}(z, v)$. The previous argument still applies, so proceeding by backward induction, from $t_{k-1}$ to $t_{k-2}, \ldots, t_{0}$, we conclude that it suffices to take the supremum in (6.19) only over $\left\{u_{j}, j \leq m\right\}$ such that each process $u_{j}$ is adapted to the filtration generated by $W_{j}$. The bound of (6.19) then becomes

$$
\mathbb{P}\left(\rho^{N} \in B(\gamma, \delta)\right) \leq\left(\begin{array}{c}
N \\
m
\end{array}\right)\left[\sup _{u_{1}, y_{1}} \mathbb{P}\left(\left|Z_{1}^{\left(u_{1}\right)}(s)-y_{1}\right| \leq \delta\right)\right]^{m},
$$

where the supremum is now over non-random $y_{1} \in \mathbb{R}$ and all [inf $\sigma$, sup $\sigma$ ]-valued processes $u_{1}$ adapted to the filtration generated by $W_{1}$. Since $m / N \geq \epsilon$ is bounded away from zero, we get (6.16) from

$$
\limsup _{\delta \downarrow 0} \sup _{u_{1}, y_{1}} \mathbb{P}\left(\left|Z_{1}^{\left(u_{1}\right)}(s)-y_{1}\right| \leq \delta\right)=0,
$$

which is an immediate consequence of the stronger result in [31, Theorem 1].

Case (b). We continue with $b \equiv 0, \gamma(0, \cdot)=\rho_{0}$, and $R^{(\gamma)} \in C_{b}\left(\mathbb{R}_{T}\right)$, whereas (1.6) fails, namely

$$
\int_{0}^{T}\left(\gamma(t),|x|^{1+\iota_{\star}}\right) \mathrm{d} t=\infty
$$

for $\iota_{\star} \in(0,1]$ of Asmp. 1.2(c). Let $0 \leq f_{K} \uparrow f_{\infty}$ be infinitely differentiable functions such that

$$
\begin{array}{r}
f_{K}(x)=|x|^{1+\iota_{\star}} \text { on }[-K,-1] \cup[1, K], \quad f_{K}(x) \leq 1 \text { on }[-1,1], \\
\left|f_{K}^{\prime}\right|^{2} \leq 8 f_{K}, \quad\left\|f_{K}\right\|_{\infty} \leq 2 K^{1+\iota_{\star}}, \quad\left\|f_{K}^{\prime}\right\|_{\infty} \leq 2 K^{\iota_{\star}}, \quad\left\|f_{K}^{\prime \prime}\right\|_{\infty} \leq 2
\end{array}
$$

(we construct $f_{K}$ by smoothing the function $(K \wedge(|x| \vee 1))^{1+\iota_{\star}}$ around the points $\{ \pm 1, \pm K\})$. Next, for $Z_{N}^{f_{K}}(t):=\left(\rho^{N}(t), f_{K}\right)$ consider the stopping times

$$
\tau_{N}^{K}(r):=\inf \left\{t \geq 0: Z_{N}^{f_{K}}(t) \geq 2 r\right\} .
$$

Since $f_{K}(x) \leq 2|x|^{1+\iota_{\star}}+1$ we have from Asmp. 1.2(c) that for some $C_{\iota_{\star}}$ finite

$$
\sup _{K, N \in \mathbb{N}}\left\{Z_{N}^{f_{K}}(0)\right\} \leq 2 \sup _{N \in \mathbb{N}}\left(\rho^{N}(0),|x|^{1+\iota_{\star}}\right)+1 \leq C_{\iota_{\star}} .
$$

With $\left\|f_{K}^{\prime \prime}\right\|_{\infty} \leq 2$ we get upon applying Itô's formula for $Z_{N}^{f_{K}}$ that, for the continuous martingale $M_{N}^{f_{K}}(t)$ of (6.3) and any $r \geq r_{0}:=C_{\iota_{\star}}+2\|A\|_{\infty} T$,

$$
\mathbb{P}\left(\tau_{N}^{K}(r) \leq T\right) \leq \mathbb{P}\left(M_{N}^{f_{K}}\left(\tau_{N}^{K}(r) \wedge T\right) \geq r\right)
$$

Further, since $\left|f_{K}^{\prime}\right|^{2} \leq 8 f_{K}$, we have for $V(\cdot)$ of (6.4) that

$$
\left\langle M_{N}^{f_{K}}\right\rangle(t)=\frac{1}{N} \int_{0}^{t} V^{f_{K}}\left(\rho^{N}\right)(s) \mathrm{d} s \leq \frac{16\|A\|_{\infty}}{N} \int_{0}^{t} Z_{N}^{f_{K}}(s) \mathrm{d} s .
$$


In particular, by the definition of $\tau_{N}^{K}(r)$,

$$
\left\langle M_{N}^{f_{K}}\right\rangle\left(\tau_{N}^{K}(r) \wedge T\right) \leq \kappa \frac{r}{N}
$$

for $\kappa=128\|A\|_{\infty} T$. Appealing to the martingale representation theorem,

$$
M_{N}^{f_{K}}\left(\tau_{N}^{K}(r) \wedge T\right) \stackrel{d}{=} \beta\left(\left\langle M_{N}^{f_{K}}\right\rangle\left(\tau_{N}^{K}(r) \wedge T\right)\right),
$$

for some standard Brownian motion $\beta$. Hence, by (6.25) and (6.26), for any $r \geq r_{0}$,

$$
\mathbb{P}\left(\tau_{N}^{K}(r) \leq T\right) \leq \mathbb{P}\left(\sup _{t \in[0, \kappa r / N]}\{\beta(t)\} \geq r\right) \leq 2 \exp \left(-\frac{N r}{2 \kappa}\right) .
$$

Since $d_{L}$ is a metric for the weak convergence in $M_{1}(\mathbb{R})$ and $f_{K} \in C_{b}(\mathbb{R})$, by dominated convergence the functionals $G_{K}(\xi):=\int_{0}^{T}\left(\xi(t), f_{K}\right) \mathrm{d} t$ on $\mathcal{C}$ are continuous with respect to the distance $d(\cdot, \cdot)$ of (1.5). Consequently, for any $K \in \mathbb{N}$, there exists $\delta_{K}>0$ such that

$$
\rho \in B\left(\gamma, \delta_{K}\right) \quad \Longrightarrow \quad G_{K}(\rho) \geq \frac{1}{2} G_{K}(\gamma)
$$

Further, if $G_{K}\left(\rho^{N}\right) \geq 2 T r$, then necessarily $\tau_{N}^{K}(r) \leq T$. Thus, setting $r_{K}=\frac{1}{4 T} G_{K}(\gamma)$, we have, for any $K$ and $\delta \leq \delta_{K}$, that

$$
\mathbb{P}\left(\rho^{N} \in B(\gamma, \delta)\right) \leq \mathbb{P}\left(G_{K}\left(\rho^{N}\right) \geq 2 T r_{K}\right) \leq \mathbb{P}\left(\tau_{N}^{K}\left(r_{K}\right) \leq T\right),
$$

which, in view of (6.27), yields the bound

$$
\lim _{\delta \downarrow 0} \limsup _{N \rightarrow \infty} \frac{1}{N} \log \mathbb{P}\left(\rho^{N} \in B(\gamma, \delta)\right) \leq-\frac{G_{K}(\gamma)}{8 \kappa T}
$$

provided $G_{K}(\gamma) \geq 4 T r_{0}$. Our assumption (6.23) and the fact that $f_{K}(x)=|x|^{1+\iota_{\star}}$ for all $|x| \in[1, K]$ imply that $G_{K}(\gamma) \rightarrow \infty$ as $K \rightarrow \infty$, thereby establishing (6.16).

Case (c). Steps 1 and 2 of the proof of Prop. 6.1 require only that $\gamma(0)=\rho_{0}$ and $R^{(\gamma)} \in C_{b}\left(\mathbb{R}_{T}\right)$, both of which hold here. Hence, in view of the derivation leading to (6.13), we get (6.16) as soon as

$$
\sup _{g \in \overline{\mathcal{S}}} I^{g}((\gamma, g))=\infty,
$$

for $I^{g}(\cdot)$ of (6.15). Further, $I^{g}((\gamma, g))$ is finite only if the identity

$$
(\gamma, g)(t)=(\Psi h)(t)=(\gamma, g)(0)+\bar{H}^{g}(\gamma)(t)+h\left(\bar{V}^{g}(\gamma)(t)\right)
$$

holds for some $h$ absolutely continuous. By assumption, there exists $g \in \overline{\mathcal{S}}$ for which the l.h.s. of (6.29) is not absolutely continuous on $[0, T]$. In contrast, both $\bar{H}^{g}(\gamma)(\cdot)$ and $\bar{V}^{g}(\gamma)(\cdot)$ are absolutely continuous for any $g$ and $\gamma$, hence so is the r.h.s. of (6.29) for any absolutely continuous $h$, resulting with (6.28).

We conclude this section by showing the exponential tightness of $\left\{\rho^{N}, N \in \mathbb{N}\right\}$.

Proposition 6.3. Under Asmp. 1.2(a)-(c), the sequence $\left\{\rho^{N}\right\}$ is exponentially tight on $\mathcal{C}$. That is, for any finite $M$ there exists a compact $K_{M} \subset \mathcal{C}$ for which

$$
\limsup _{N \rightarrow \infty} \frac{1}{N} \log \mathbb{P}\left(\rho^{N} \notin K_{M}\right) \leq-M .
$$


Proof. In view of (6.12) it suffices to confirm the criterion for exponential tightness given in [9, Lemma A.2]. Specifically, this amounts to showing as Step 1 that $\left\{\rho^{N}(t), N \in \mathbb{N}\right\}$ is exponentially tight on $\left(M_{1}(\mathbb{R}), d_{L}\right)$ for each fixed $t \in[0, T]$ (rational), and then proving as Step 2 that, for any fixed $\epsilon>0$, one has

$$
\lim _{\kappa \downarrow 0} \sup _{N \in \mathbb{N}} \frac{1}{N} \log \mathbb{P}\left(\sup _{0 \leq s, t \leq T,|t-s| \leq \kappa} d_{L}\left(\rho^{N}(s), \rho^{N}(t)\right)>\delta\right)=-\infty .
$$

Step 1. By Prokhorov's theorem, the set

$$
\left\{\alpha \in M_{1}(\mathbb{R}):(\alpha, \phi) \leq C\right\},
$$

is pre-compact in $\left(M_{1}(\mathbb{R}), d_{L}\right)$ for $\phi(x)=|x|$ and any $C$ finite. Hence, to prove our first assertion, it suffices to show that for some $C=C(M, T)<\infty$

$$
\limsup _{N \rightarrow \infty} \frac{1}{N} \log \sup _{t \in[0, T]} \mathbb{P}\left(\left(\rho^{N}(t), \phi\right)>2 C\right) \leq-M .
$$

To this end, from (6.24) we have that $\sup _{N}\left(\rho^{N}(0), \phi\right) \leq C_{0}$ finite, hence with

$$
Z_{i}(t)=\int_{0}^{t} \sigma\left(F_{\rho^{N}(r)}\left(X_{i}(r)\right)\right) \mathrm{d} W_{i}(r)
$$

it follows by Markov's inequality, that for any $C \geq C_{0}+T\|b\|_{\infty}$

$$
\begin{aligned}
\mathbb{P}\left(\left(\rho^{N}(t), \phi\right)>2 C\right) & =\mathbb{P}\left(\sum_{i=1}^{N}\left|X_{i}(t)\right|>2 C N\right) \\
& \leq \mathbb{P}\left(\sum_{i=1}^{N}\left|Z_{i}(t)\right|>C N\right) \leq e^{-C N} \sup _{\left\{u_{j}\right\}} \mathbb{E}\left[\prod_{j=1}^{N} e^{\left|Z_{j}^{\left(u_{j}\right)}(t)\right|}\right] .
\end{aligned}
$$

The supremum here is over the same collection of simple adapted processes $\left\{u_{j}\right\}$ we considered in (6.21) and with $Z_{j}^{\left(u_{j}\right)}(t)$ as in (6.20). By the same argument we have used en-route to (6.22), it suffices to consider the situation where each process $u_{j}$ is adapted to the filtration generated by the Brownian motion $W_{j}$. Consequently, the preceding upper bound simplifies to

$$
\mathbb{P}\left(\left(\rho^{N}(t), \phi\right)>2 C\right) \leq e^{-C N} \sup _{u_{1}} \mathbb{E}\left[e^{\left|Z_{1}^{\left(u_{1}\right)}(t)\right|}\right]^{N},
$$

where the supremum is now over all $[\inf \sigma, \sup \sigma]$-valued processes $u_{1}$ adapted to the filtration generated by $W_{1}$. Viewing such process $Z_{1}^{\left(u_{1}\right)}(t)$ as a time changed standard Brownian motion $\beta$, results with

$$
\sup _{u_{1}} \mathbb{E}\left[e^{\left|Z_{1}^{\left(u_{1}\right)}(t)\right|}\right] \leq \mathbb{E}\left[\exp \left(\sup _{r \in\left[0,2 t\|A\|_{\infty}\right]}|\beta(r)|\right)\right]<\infty,
$$

which together with (6.35) yields (6.32).

Step 2. First note that for $d_{B L}(\cdot, \cdot)$ of $(\underline{6.12}), Z_{i}(t)$ of (6.33) and any $s, t \in[0, T]$

$$
\begin{aligned}
& \frac{1}{2} d_{L}\left(\rho^{N}(s), \rho^{N}(t)\right)^{2} \leq d_{B L}\left(\rho^{N}(s), \rho^{N}(t)\right) \\
& \quad \leq \frac{1}{N} \sum_{i=1}^{N}\left|X_{i}(t)-X_{i}(s)\right| \leq \frac{1}{N} \sum_{i=1}^{N}\left|Z_{i}(t)-Z_{i}(s)\right|+|t-s|\|b\|_{\infty}
\end{aligned}
$$


(see [12, proof of Theorem 11.3.3] for the left-most inequality). Thus, we have (6.31), as soon as we show that, for

$$
\operatorname{osc}(Z ; \kappa, T)=\sup _{0 \leq s, t \leq T,|t-s| \leq \kappa}\{|Z(t)-Z(s)|\}
$$

and any fixed $\lambda, \delta>0$,

$$
\limsup _{\kappa \downarrow 0} \frac{1}{N} \log \mathbb{P}\left(\sum_{i=1}^{N} \operatorname{osc}\left(Z_{i} ; \kappa, T\right)>\delta N\right) \leq-\lambda \delta .
$$

As for the proof of (6.37), similarly to the derivation of (6.34) and (6.35), by Markov's inequality we have that

$$
\begin{aligned}
\mathbb{P}\left(\sum_{i=1}^{N} \operatorname{osc}\left(Z_{i} ; \kappa, T\right)>\delta N\right) & \leq e^{-\lambda \delta N} \sup _{\left\{u_{j}\right\}} \mathbb{E}\left[\prod_{j=1}^{N} e^{\lambda \operatorname{osc}\left(Z_{j}^{\left(u_{j}\right)} ; \kappa, T\right)}\right] \\
& \leq e^{-\lambda \delta N}\left(\sup _{u_{1}} \mathbb{E}\left[e^{\lambda \operatorname{osc}\left(Z_{1}^{\left(u_{1}\right)} ; \kappa, T\right)}\right]\right)^{N}
\end{aligned}
$$

where the latter supremum is over all $[\inf \sigma, \sup \sigma]$-valued processes $u_{1}$ adapted to the filtration generated by $W_{1}$. Further, viewing the continous martingale $Z_{1}^{\left(u_{1}\right)}(t)$ as a time-changed standard Brownian motion $\beta$, it follows by the same reasoning we applied in Step 1 that

$$
\sup _{u_{1}} \mathbb{E}\left[e^{\lambda \operatorname{osc}\left(Z_{1}^{\left(u_{1}\right)} ; \kappa, T\right)}\right] \leq \mathbb{E}\left[e^{\lambda \operatorname{osc}\left(\beta ; 2\|A\|_{\infty} \kappa, 2\|A\|_{\infty} T\right)}\right]
$$

Now, since

$$
\operatorname{osc}\left(\beta ; 2\|A\|_{\infty} \kappa, 2\|A\|_{\infty} T\right) \leq 2 \sup _{r \in\left[0,2\|A\|_{\infty} T\right]}\{|\beta(r)|\}
$$

which has finite exponential moments of all order, by sample path continuity of $\beta$ and dominated convergence, the r.h.s. of (6.39) decays to one as $\kappa \downarrow 0$. Thus, combining (6.39) and (6.38) results with (6.37), thereby completing the proof of the proposition.

\section{Proof of Proposition 2.7}

We work throughout under parts (a)-(c) of Asmp. 1.2 with $\iota_{\star}=1$.

Proof of Part (a). Recall from [2, Section 3] that in any solution of (1.1) the ordered particles $X_{(1)}(t) \leq X_{(2)}(t) \leq \cdots \leq X_{(N)}(t)$ satisfy the SDS

$$
\mathrm{d} X_{(j)}(t)=b_{j} \mathrm{~d} t+\sigma_{j} \mathrm{~d} \beta_{j}(t)+\frac{1}{2} \mathrm{~d} \Lambda_{j-1}(t)-\frac{1}{2} \mathrm{~d} \Lambda_{j}(t), \quad j=1, \ldots, N,
$$

for independent standard Brownian motions $\left\{\beta_{j}\right\}$ where $\Lambda_{0}(t)=\Lambda_{N}(t) \equiv 0$ and $\Lambda_{j}(t)$ for $j=1, \ldots, N-1$ denotes the local time at zero accumulated by the $\mathbb{R}_{+}$-valued path $X_{(j+1)}(\cdot)-X_{(j)}(\cdot)$ by time $t$. It is also shown in [2, Section 3] that strong existence and uniqueness holds for the SDS (7.1). We claim that w.p.1

$$
t \mapsto \Delta_{N}(t):=\frac{1}{N} \sum_{j=1}^{N}\left|X_{(j)}(t)-\tilde{X}_{(j)}(t)\right|,
$$

is non-increasing, for any two strong solutions $X$ and $\tilde{X}$ of (7.1) driven by the same Brownian motions $\left\{\beta_{j}\right\}$ (extending [23, inequality (15)] to arbitrary initial conditions). Indeed, since $t \mapsto\left(X_{(j)}(t)-\widetilde{X}_{(j)}(t)\right)$ is of bounded variation, its local time process at 
zero vanishes. Thus, setting $S_{j}(t):=\operatorname{sgn}\left(X_{(j)}(t)-\widetilde{X}_{(j)}(t)\right)$, we have by Tanaka's formula, followed by summation by parts, that

$$
\begin{aligned}
\mathrm{d} \Delta_{N}(t) & =\frac{1}{2 N} \sum_{j=1}^{N} S_{j}(t)\left(\mathrm{d} \Lambda_{j-1}(t)-\mathrm{d} \Lambda_{j}(t)-\mathrm{d} \widetilde{\Lambda}_{j-1}(t)+\mathrm{d} \widetilde{\Lambda}_{j}(t)\right) \\
& =\frac{1}{2 N} \sum_{j=2}^{N}\left(S_{j}(t)-S_{j-1}(t)\right)\left(\mathrm{d} \Lambda_{j-1}(t)-\mathrm{d} \widetilde{\Lambda}_{j-1}(t)\right) .
\end{aligned}
$$

Since $X_{(j)}(t)=X_{(j-1)}(t)$ at times of increase of $\Lambda_{j-1}(t)$ and $\tilde{X}_{(j)}(t)=\tilde{X}_{(j-1)}(t)$ at times of increase of $\widetilde{\Lambda}_{j-1}(t)$, it is easy to check that, for $j=2, \ldots, N$ and all $t \geq 0$,

$$
\left(S_{j}(t)-S_{j-1}(t)\right) \mathrm{d} \Lambda_{j-1}(t) \leq 0 \leq\left(S_{j}(t)-S_{j-1}(t)\right) \mathrm{d} \widetilde{\Lambda}_{j-1}(t) .
$$

Hence, by (7.3) we have, as claimed, that $\mathrm{d} \Delta_{N}(t) \leq 0$. Next, with $\rho^{N}$ and $\widetilde{\rho}^{N}$ denoting the paths of empirical measures of $X$ and $\widetilde{X}$, respectively, we see that for any $t \geq 0$,

$$
d_{B L}\left(\rho^{N}(t), \widetilde{\rho}^{N}(t)\right) \leq \Delta_{N}(t) \leq \Delta_{N}(0)=W_{1}\left(\rho^{N}(0), \widetilde{\rho}^{N}(0)\right),
$$

where $W_{1}(\cdot, \cdot)$ stands for the $L_{1}$-Wasserstein distance on $M_{1}(\mathbb{R})$ :

$$
W_{1}\left(\alpha_{1}, \alpha_{2}\right):=\inf \left\{\mathbb{E}\left[\left|Y_{1}-Y_{2}\right|\right]: Y_{1} \sim \alpha_{1}, Y_{2} \sim \alpha_{2}\right\} .
$$

Further, as $\frac{1}{2} d_{L}\left(\alpha_{1}, \alpha_{2}\right)^{2} \leq d_{B L}\left(\alpha_{1}, \alpha_{2}\right)$ (see (6.36) $)$, it follows from (17.4) that

$$
\frac{1}{2} d\left(\rho^{N}, \widetilde{\rho}^{N}\right)^{2} \leq W_{1}\left(\rho^{N}(0), \widetilde{\rho}^{N}(0)\right) .
$$

Fixing $\ell, \epsilon$ we let $\rho^{N, \ell, \epsilon}(0)$ be the empirical measure of $\widetilde{X}_{(j)}(0)=F^{-1}\left(u_{j, N}\right)$, with $u_{j, N}=j /(N+1)$ and $F:=F_{\gamma^{\ell, \epsilon}(0)}$ a continuous CDF. By the preceding, we get (2.15) upon showing that

$$
\limsup _{\epsilon \rightarrow 0} \limsup _{\ell \rightarrow \infty} \limsup _{N \rightarrow \infty} W_{1}\left(\rho^{N}(0), \rho^{N, \ell, \epsilon}(0)\right)=0 .
$$

We then complete the proof of part (a) by observing that,

$$
\begin{aligned}
\frac{N}{N+1}\left(|x|, \rho^{N, \ell, \epsilon}(0)\right) & =\frac{1}{N+1} \sum_{j=1}^{N}\left|F^{-1}\left(u_{j, N}\right)\right| \\
& \leq \int_{0}^{1}\left|F^{-1}(u)\right| \mathrm{d} u=\left(|x|, \gamma^{\ell, \epsilon}(0)\right),
\end{aligned}
$$

which is finite since $\gamma^{\ell, \epsilon}(0) \in M_{1}^{(0)}(\mathbb{R})$, whereas for $N \rightarrow \infty$ and any fixed $x \in \mathbb{R}$,

$$
\rho^{N, \ell, \epsilon}(0)((-\infty, x])=\frac{1}{N}\lfloor(N+1) F(x)\rfloor \rightarrow F(x) .
$$

That is, as claimed our $\left\{\rho^{N, \ell, \epsilon}(0)\right\}$ satisfy Asmp. 1.2(c) (with $\iota_{\star}=0$ ). In particular,

$$
\lim _{N \rightarrow \infty} d_{B L}\left(\rho^{N, \ell, \epsilon}(0), \gamma^{\ell, \epsilon}(0)\right)=0 .
$$

Turning to prove (7.5), recall that, for any $\kappa \geq 1$,

$$
W_{1}\left(\alpha_{1}, \alpha_{2}\right) \leq \kappa d_{B L}\left(\alpha_{1}, \alpha_{2}\right)+3\left(|x| \mathbf{1}_{|x|>\kappa}, \alpha_{1}+\alpha_{2}\right)
$$

(for example, this follows from [12, Theorem 11.8.2]). From Asmp. 1.2(c) we know that $d_{B L}\left(\rho^{N}(0), \gamma(0)\right) \rightarrow 0$ when $N \rightarrow \infty$, whereas $d_{B L}\left(\gamma(0), \gamma^{\ell, \epsilon}(0)\right) \rightarrow 0$ when $\ell \rightarrow \infty$ 
followed by $\epsilon \rightarrow 0$ (see (2.11)). Combining these facts with (7.7), (7.8), and the triangle inequality for $d_{B L}(\cdot, \cdot)$, we get (7.5) by showing that

$$
\begin{array}{r}
\lim _{\kappa \rightarrow \infty} \limsup _{N \rightarrow \infty}\left(|x| \mathbf{1}_{|x|>\kappa}, \rho^{N}(0)\right)=0, \\
\lim _{\kappa \rightarrow \infty} \limsup _{\epsilon \rightarrow 0} \limsup _{\ell \rightarrow \infty} \limsup _{N \rightarrow \infty}\left(|x| \mathbf{1}_{|x|>\kappa}, \rho^{N, \ell, \epsilon}(0)\right)=0 .
\end{array}
$$

With $\sup _{N}\left(|x|^{2}, \rho^{N}(0)\right)$ finite (see Asmp. 1.2(c), $\iota_{\star}=1$ ), we obviously have (7.9)). As for (7.10), from (7.7) and $\gamma^{\ell, \epsilon}(0)$ having a density, we deduce that as $N \rightarrow \infty$

$$
\left(|x| \mathbf{1}_{|x| \leq \kappa}, \rho^{N, \ell, \epsilon}(0)\right) \rightarrow\left(|x| \mathbf{1}_{|x| \leq \kappa}, \gamma^{\ell, \epsilon}(0)\right) .
$$

Further, when $\ell \rightarrow \infty, \epsilon \rightarrow 0$, and then $\kappa \rightarrow \infty$, we have by (2.10) that

$$
\left(|x| \mathbf{1}_{|x|>\kappa}, \gamma^{\ell, \epsilon}(0)\right) \leq \frac{1}{\ell}\left(|x|, \widehat{\mu}^{\epsilon}\right)+\frac{1}{\kappa}\left(|x|^{2}, \gamma^{\epsilon}(0)\right) \rightarrow 0,
$$

which together with (7.6) yields (7.10).

Proof of Part (b). From part (a) we know that Asmp. 1.2(c) holds for $\iota_{\star}=0$, $\rho^{N, \ell, \epsilon}(0)$, and $\rho_{0}^{\ell, \epsilon}=\gamma^{\ell, \epsilon}(0)$, so we simplify notation by dropping hereafter the superscripts $\ell, \epsilon$. That is, we fix $\gamma \in \mathcal{G}$ with $\widetilde{J}(\gamma)<\infty$ (see Definition 2.4 and (1.8), respectively), having $R=R^{(\gamma)} \in C_{b}^{\infty}\left(\mathbb{R}_{T}\right) \bigcap \mathcal{F}_{3 / 2}$ (for $\mathcal{F}_{q}$ of (1.7)), starting at $R(0, x)=F_{\rho_{0}}(x)$, with $R_{x}$ strictly positive on $\mathbb{R}_{T}$ and such that

$$
h=\frac{R_{t}-\left(A(R) R_{x}\right)_{x}}{A(R) R_{x}} \in C_{b}\left(\mathbb{R}_{T}\right)
$$

with $x \mapsto h(t, x)$ uniformly Lipschitz continuous on $\mathbb{R}_{T}$. The functional on $\mathcal{C}$

$$
\widehat{J}(\rho)=\frac{1}{2} \int_{0}^{T}\left(\rho, U(\rho)^{2}\right)(s) \mathrm{d} s,
$$

with the continuous in time, bounded function on $\mathbb{R}_{T}$

$$
U(\rho):=\frac{h A\left(R^{(\rho)}\right)+b\left(R^{(\rho)}\right)}{\sigma\left(R^{(\rho)}\right)},
$$

is then such that $\widetilde{J}(\gamma)=\widehat{J}(\gamma)<\infty$.

To prove the local large deviations lower bound (2.16) we introduce in Step 1 a suitable change of measure to $\mathbb{Q}_{1}$ for which the relevant Radon-Nikodym derivative is at least $e^{-N(\widehat{J}(\gamma)+\epsilon)}$ on the event $\left\{\rho^{N} \in B(\gamma, \delta)\right\}$ (when $N \rightarrow \infty$ followed by $\delta \downarrow 0$ ), then verify in Step 2 the relevant LLN (7.16) for $\rho^{N}$ under $\mathbb{Q}_{1}$. This step relies on proving in Lemma 7.1 that any limit point $\widehat{\gamma}$ of $\rho^{N}$ has no atoms, from which we deduce that the corresponding path of CDF-s $w=R^{(\widehat{\gamma})}$ solves the weak form (7.18) of the porus medium equation (1.10). The tilt $h$ in the latter equation is given by (7.11), so $R^{(\gamma)}$ is one such solution, and the analysis of Lemma 7.2 guarantees its uniqueness.

Step 1. Since $x \mapsto h(t, x)$ is uniformly bounded, Lipschitz function on $\mathbb{R}_{T}$, there exists, for any $q \in \mathbb{R}$, a probability measure $\mathbb{Q}_{q}$ under which, for $i=1, \ldots, N$,

$$
X_{i}(t)=X_{i}(0)+\int_{0}^{t} \psi_{q}\left(\rho^{N}\right)\left(s, X_{i}(s)\right) \mathrm{d} s+\int_{0}^{t} \sigma\left(F_{\rho^{N}(s)}\left(X_{i}(s)\right)\right) \mathrm{d} W_{i}^{q}(s),
$$

with $\psi_{q}(\rho)=b\left(R^{(\rho)}\right)-q U(\rho) \sigma\left(R^{(\rho)}\right)$ and $\left\{W_{i}^{q}\right\}$ independent standard Brownian motions. Further, $\mathbb{P}=\mathbb{Q}_{0}$ and, similarly to (6.17) (which has $h \equiv 0$, i.e. $\psi_{q}=$ 
$\left.(1-q) b\left(R^{(\rho)}\right)\right)$, by Girsanov's theorem

$$
\frac{\mathrm{d} \mathbb{Q}_{q}}{\mathrm{~d} \mathbb{P}}=\exp \left(-q M_{N}(T)-\frac{q^{2}}{2}\left\langle M_{N}\right\rangle(T)\right)
$$

with the continuous martingales

$$
M_{N}(t)=\sum_{i=1}^{N} \int_{0}^{t} U\left(\rho^{N}\right)\left(s, X_{i}(s)\right) \mathrm{d} W_{i}^{0}(s)
$$

satisfying $\left\langle M_{N}\right\rangle(T)=2 N \widehat{J}\left(\rho^{N}\right)$ (see [24, Theorem 3.5.1]). By the triangle inequality, for any $\rho \in B(\gamma, \delta)$,

$$
\begin{aligned}
2|\widehat{J}(\rho)-\widehat{J}(\gamma)| & \leq \sup _{\rho \in B(\gamma, \delta)}\left|\int_{0}^{T}\left(\rho, U(\rho)^{2}-U(\gamma)^{2}\right)(s) \mathrm{d} s\right| \\
& +\sup _{\rho \in B(\gamma, \delta)}\left|\int_{0}^{T}\left(\rho, U(\gamma)^{2}\right)(s) \mathrm{d} s-\int_{0}^{T}\left(\gamma, U(\gamma)^{2}\right)(s) \mathrm{d} s\right|
\end{aligned}
$$

Since $b$ and $\sigma$ are bounded, Lipschitz functions, and $h, \sigma^{-1}$ are uniformly bounded, it is easy to check that $U(\rho)^{2}$ is a bounded, Lipschitz function of $R^{(\rho)}$. Consequently, $\left|U(\rho)^{2}-U(\gamma)^{2}\right| \leq C\left|R^{(\rho)}-R^{(\gamma)}\right|$ for some finite $C$ and all $(t, x) \in \mathbb{R}_{T}$. Thus, from (6.8) it follows that the first term on the r.h.s. of (17.14) converges to 0 as $\delta \downarrow 0$. Further, with $h$ and $R^{(\gamma)}$ in $C_{b}\left(\mathbb{R}_{T}\right)$, also $U(\gamma)^{2} \in C_{b}\left(\mathbb{R}_{T}\right)$. Hence, by dominated convergence, the functional $\xi \mapsto \int_{0}^{T}\left(\xi, U(\gamma)^{2}\right)(s) \mathrm{d} s$ is continuous on $\mathcal{C}$, and the second term on the r.h.s. of (7.14) also converges to 0 as $\delta \downarrow 0$. We conclude that, for any fixed $\epsilon>0$ and all $\delta<\delta_{0}(\epsilon)$,

$$
\rho^{N} \in B(\gamma, \delta) \quad \Longrightarrow \quad\left|\frac{1}{2}\left\langle M_{N}\right\rangle(T)-N \widehat{J}(\gamma)\right| \leq \epsilon N .
$$

For such $\delta<\delta_{0}(\epsilon)$, any $p=q /(q-1)>1, q>1$, and all $N \in \mathbb{N}$, we thus have by Hölder's inequality that

$$
\begin{aligned}
& \mathbb{Q}_{1}\left(\rho^{N} \in B(\gamma, \delta)\right)=\mathbb{P}\left[e^{-M_{N}(T)-\frac{1}{2}\left\langle M_{N}\right\rangle(T)} \mathbf{1}_{\left\{\rho^{N} \in B(\gamma, \delta)\right\}}\right] \\
& \leq \mathbb{P}\left[e^{-q M_{N}(T)-\frac{q}{2}\left\langle M_{N}\right\rangle(T)} \mathbf{1}_{\left\{\rho^{N} \in B(\gamma, \delta)\right\}}\right]^{1 / q} \mathbb{P}\left(\rho^{N} \in B(\gamma, \delta)\right)^{1 / p} \\
& \leq e^{(q-1) N(\widehat{J}(\gamma)+\epsilon)} \mathbb{P}\left[e^{-q M_{N}(T)-\frac{q^{2}}{2}\left\langle M_{N}\right\rangle(T)}\right]^{1 / q} \mathbb{P}\left(\rho^{N} \in B(\gamma, \delta)\right)^{1 / p} \\
& =e^{(q-1) N(\widehat{J}(\gamma)+\epsilon)}\left[\mathbb{Q}_{q}(1)\right]^{1 / q} \mathbb{P}\left(\rho^{N} \in B(\gamma, \delta)\right)^{1 / p} .
\end{aligned}
$$

Consider $\frac{p}{N} \log (\cdot)$ of both sides, taking first $N \rightarrow \infty$ followed by $\delta \downarrow 0$, to find that

$$
\lim _{\delta \downarrow 0} \liminf _{N \rightarrow \infty} \frac{1}{N} \log \mathbb{P}\left(\rho^{N} \in B(\gamma, \delta)\right) \geq-q(\widehat{J}(\gamma)+\epsilon),
$$

for any $\epsilon>0, q>1$ (hence (2.16) holds), provided that

$$
\lim _{N \rightarrow \infty} \mathbb{Q}_{1}\left(\rho^{N} \in B(\gamma, \delta)\right)=1 .
$$

Step 2. To prove (7.16), recall that $\mathbb{Q}_{1}$ corresponds to $\left\{X_{i}(t)\right\}$ of $(7.13)$ for drift $\psi_{1}(\rho)=-h A\left(R^{(\rho)}\right)$ and, for each $N \in \mathbb{N}$, let $Q_{N}$ denote the law of $\rho^{N}$ under $\mathbb{Q}_{1}$. One then deduces the uniform tightness, and hence pre-compactness, of the collection $\left\{Q_{N}\right\}$ in the space of probability measures on $\mathcal{C}$. Indeed, Steps 1 and 2 of the proof of [36. Theorem 1.1] rely only on a general compactness criterion for subsets of $\mathcal{C}$ (taken from [17, Lemma 1.3]), so can be carried out mutatis mutandis, appealing here to boundedness of the drift and diffusion coefficients in (7.13) (in place of boundedness of 
the corresponding coefficients in the dynamics treated in [36, Theorem 1.1]). Moreover, since $\rho^{N}(0) \rightarrow \rho_{0}=\gamma(0, \cdot)$, the computations there which involve the initial conditions, can be omitted here. To prove (7.16), it thus suffices to show that the atomic measure $\delta_{\gamma}$ is the only possible limit point of the sequence $\left\{Q_{N}\right\}$. Alternatively, passing to the relevant sub-sequence and utilizing the Skorokhod representation theorem in the form of [13, Theorem 3.5.1], we can and shall assume that the variables $\rho^{N}, N \in \mathbb{N}$ are defined on the same probability space and converge almost surely in $\mathcal{C}$, when $N \rightarrow \infty$, to some limiting variable $\widehat{\gamma}$. Thus, the task of proving (17.16) amounts to showing that $\widehat{\gamma}=\gamma$ w.p.1.

To this end, fixing $g \in \overline{\mathcal{S}}$ and replacing hereafter $b\left(R^{(\xi)}\right)$ by $-h A\left(R^{(\xi)}\right)$ in the definition of $\mathcal{R}^{\xi} \mathrm{g}$, note that the l.h.s. of the identity (6.2) converges w.p.1 as $N \rightarrow \infty$, to $(\widehat{\gamma}, g)(t)-\left(\rho_{0}, g(0)\right)$, for all $t \in[0, T]$. We claim that w.p.1 the r.h.s. of (6.2) converges to $\int_{0}^{t} H^{g}(\widehat{\gamma})(s) \mathrm{d} s$ and consequently for all $t \in[0, T]$,

$$
(\widehat{\gamma}, g)(t)-\left(\rho_{0}, g(0)\right)=\int_{0}^{t}\left(\widehat{\gamma}, g_{t}+\left(g_{x x}-h g_{x}\right) A\left(R^{(\widehat{\gamma})}\right)\right)(s) \mathrm{d} s .
$$

Indeed, recall that $\bar{V}^{g}\left(\rho^{N}\right)(T)$ is uniformly bounded by $C=2 T\left\|A g_{x}^{2}\right\|_{\infty}$ finite, so $\left\langle M_{N}^{g}\right\rangle(T) \leq C / N$ and w.p.1 the continuous martingale $M_{N}^{g}(t) \rightarrow 0$ uniformly over $[0, T]$ (for example, combine the Burkholder-Davis-Gundy inequalities, see [24, Theorem 3.3.28], and the Borel-Cantelli lemma). Further, we show in Lemma [7.1 that w.p.1. $R^{(\widehat{\gamma})} \in C_{b}\left(\mathbb{R}_{T}\right)$, from which one deduces as in the derivation of (6.8), that w.p.1

$$
\lim _{N \rightarrow \infty} \int_{0}^{T}\left(\rho^{N},\left|F_{\rho^{N}}-R^{(\widehat{\gamma})}\right|\right)(s) \mathrm{d} s=0 .
$$

Since $R^{(\widehat{\gamma})} \in C_{b}\left(\mathbb{R}_{T}\right)$, also $\mathcal{R}^{\widehat{\gamma}} g \in C_{b}\left(\mathbb{R}_{T}\right)$. Thus, with $h g_{x}$ and $g_{x x}$ bounded, the preceding convergence to zero implies, as in the derivation of (6.10), that w.p.1

$$
\lim _{N \rightarrow \infty} \int_{0}^{T}\left|H^{g}\left(\rho^{N}\right)(s)-H^{g}(\widehat{\gamma})(s)\right| \mathrm{d} s=0,
$$

thereby completing the proof of (17.17). Now, setting $w=R^{(\widehat{\gamma})}$, and having (7.17) hold w.p.1 for all $g$ in a countable dense subset of $\overline{\mathcal{S}}$, we deduce that (17.17) holds for all $g \in \overline{\mathcal{S}}$, which amounts after integration by parts over $\mathbb{R}$ to

$$
\int_{\mathbb{R}}(f w)(t, x) \mathrm{d} x-\int_{\mathbb{R}}(f w)(0, x) \mathrm{d} x=\int_{\mathbb{R}_{t}}\left[f_{t} w+f_{x x} \Sigma(w)-(h f)_{x} \Sigma(w)\right] \mathrm{d} m,
$$

holding for $w(0, x)=F_{\rho_{0}}(x)$ and all $f=g_{x} \in \overline{\mathcal{S}}_{x}$. Here $\Sigma(w)=\int_{0}^{w} A(r) \mathrm{d} r$ and $h_{x}$ is well-defined ( $m$-a.e.), since $x \mapsto h(t, x)$ is a Lipschitz function. Note that, for $w \in C^{1,2}\left(\mathbb{R}_{T}\right.$ ), further integration by parts (to eliminate all derivatives of $f$ ) confirms that this is equivalent to $w$ solving the porous medium equation (1.10) (with initial condition $F_{\rho_{0}}$ ). In view of (7.11) one such solution is $w=R^{(\gamma)}$, hence w.p.1 $\widehat{\gamma}=\gamma$ provided we establish the uniqueness of such generalized solution. In conclusion, all that remains for establishing Prop. 2.7 is to prove the following two lemmas.

Lemma 7.1. Consider the unique weak solution of (7.13) for $\psi_{1}(\rho)=-h A\left(R^{(\rho)}\right)$, and suppose that $\widehat{\gamma}$ is an a.s. limit point in $\mathcal{C}$ of $\rho^{N}$. Then, w.p. 1 the probability measures $\widehat{\gamma}(t, \cdot)$ have no atoms for all $t \in[0, T]$.

Lemma 7.2. Suppose $h \in C_{b}\left(\mathbb{R}_{T}\right)$ with $x \mapsto h(t, x)$ uniformly Lipschitz on $\mathbb{R}_{T}$, for which (1.10) has a bounded classical solution $R=u \in C^{1,2}\left(\mathbb{R}_{T}\right)$ with $u(0, x)=F_{\rho_{0}}(x)$. 
It is then the only solution $w \in C_{b}\left(\mathbb{R}_{T}\right)$ of (7.18) with such initial conditions, for which $w(t, \cdot)$ are $\mathrm{CDF}-s$ of some path in $\mathcal{C}$.

Proof of Lemma 7.1. When proving Prop. 6.2, we first removed the $\operatorname{drift} b\left(R^{(\rho)}\right)$ thanks to the bound (6.18), which applies for any uniformly bounded drift. So, using the same argument for the measure $\mathbb{Q}_{1}$ with bounded drift $-h A\left(R^{(\rho)}\right)$, we conclude as in case (a) of the proof of Prop. 6.2 that, for any $\epsilon>0$ and some $\delta=\delta(\epsilon) \in(0, \epsilon)$,

$$
\limsup _{N \rightarrow \infty} \frac{1}{N} \log \sup _{s \in[0, T], y \in \mathbb{R}} \mathbb{Q}_{1}\left(\rho^{N}(s)([y-3 \delta, y+3 \delta]) \geq \epsilon\right) \leq-1 .
$$

Similarly, Step 1 and Step 2 of the proof of Prop. 6.3 apply whenever the drift is uniformly bounded, hence, for any $\delta, \epsilon>0$, some $M=M(\epsilon)$ finite, and $\kappa(\delta)>0$,

$$
\begin{aligned}
& \limsup _{N \rightarrow \infty} \frac{1}{N} \log \sup _{s \in[0, T]} \mathbb{Q}_{1}\left(\rho^{N}(s)([-M, M]) \leq 1-\epsilon\right) \leq-1, \\
& \limsup _{N \rightarrow \infty} \frac{1}{N} \log \mathbb{Q}_{1}\left(\sup _{0 \leq s, t \leq T,|t-s| \leq \kappa} d_{L}\left(\rho^{N}(s), \rho^{N}(t)\right)>\delta\right) \leq-1
\end{aligned}
$$

(see (6.32) and (6.31), respectively). Fixing $\delta(\epsilon)>0$ and $M(\epsilon)$ finite, then $\kappa(\delta)>0$, consider (7.19) and (7.20) at all $\left\{s_{i}, i \leq m\right\}$ on a finite $\kappa$-net in $[0, T]$ and all $\left\{y_{j}, j \leq \ell\right\}$ in a finite $\delta$-net within $[-(M+2 \delta),(M+2 \delta)]$ to conclude by (7.21) and the BorelCantelli lemma that w.p.1 for all $N$ large enough,

$$
\begin{aligned}
& \sup _{1 \leq i \leq m} \sup _{y \in \mathbb{R}}\left\{\rho^{N}\left(s_{i}\right)([y-2 \delta, y+2 \delta])\right\} \leq \epsilon, \\
& \sup _{t \in[0, T]} \inf _{1 \leq i \leq m}\left\{d_{L}\left(\rho^{N}\left(s_{i}\right), \rho^{N}(t)\right)\right\} \leq \delta .
\end{aligned}
$$

Consequently, as in (6.9),

$$
\sup _{t \in[0, T]} \sup _{y \in \mathbb{R}}\left\{\rho^{N}(t)([y-\delta, y+\delta])\right\} \leq 3 \epsilon .
$$

Combining this with the Portmanteau theorem, we infer that, for every $\epsilon>0$, there exists a $\delta>0$ such that for any limit point $\widehat{\gamma}$ of $\rho^{N}$, w.p.1

$$
\begin{aligned}
\sup _{t \in[0, T]} \sup _{y \in \mathbb{R}} \widehat{\gamma}(t)((y-\delta, y+\delta)) & \leq \sup _{t \in[0, T]} \sup _{y \in \mathbb{R}} \liminf _{N \rightarrow \infty} \rho^{N}(t)((y-\delta, y+\delta)) \\
& \leq \limsup _{N \rightarrow \infty} \sup _{t \in[0, T]} \sup _{y \in \mathbb{R}}\left\{\rho^{N}(t)((y-\delta, y+\delta))\right\} \leq 3 \epsilon .
\end{aligned}
$$

This shows that w.p. 1 the path of measures $\widehat{\gamma}$ has no atoms of mass at least $3 \epsilon$, and taking $\epsilon \downarrow 0$ finishes the proof of the lemma.

Proof of Lemma 7.2. First, note that if (7.18) holds for $w(t, \cdot)$ which are the CDF-s of some path in $\mathcal{C}$, and all $f \in \overline{\mathcal{S}}_{x}$, then it further holds for all $\widehat{f} \in \overline{\mathcal{S}}$. Indeed, for any such $\widehat{f}$ there exist $f_{k} \in \overline{\mathcal{S}}_{x}$ coinciding with $\widehat{f}$ on $[0, T] \times[-k, \infty)$ such that both $\sup _{k}\left\|f_{k}-\widehat{f}\right\|_{W_{1}^{1,2}\left(\mathbb{R}_{T}\right)}$ and $\sup _{k, t}\left\|f_{k}(t, \cdot)-\widehat{f}(t, \cdot)\right\|_{L^{1}(\mathbb{R})}$ are finite. Thus, with $x \mapsto h(x, t)$ uniformly bounded, globally Lipschitz on $\mathbb{R}_{T}$ and $\Sigma(w) \leq\|A\|_{\infty} w$, it follows from (3.1) that the value each side of (17.18) takes for $f_{k}$, converges as $k \rightarrow \infty$ to its value for $\widehat{f}$, thereby extending the scope of $(\underline{7.18})$ to all of $\overline{\mathcal{S}}$. The latter identity involves only $\left(f, f_{t}, f_{x}, f_{x x}\right)$, hence holds for any $f \in C_{c}^{1,2}\left(\mathbb{R}_{T}\right)$. Further, setting $\mathbb{K}_{r}=[0, t] \times[-r, r]$, the identity (7.18) applies for any $f \in C^{1,2}\left(\mathbb{K}_{r}\right)$ such that $f(s, \pm r)=0$ for all $s \in[0, t]$, 
provided one adds to its l.h.s. the boundary term

$$
\int_{0}^{t}\left[\left(\Sigma(w) f_{x}\right)\left(s, r^{-}\right)-\left(\Sigma(w) f_{x}\right)\left(s,-r^{-}\right)\right] \mathrm{d} s
$$

(which takes into account the jump-discontinuity of $f_{x}$ at $\partial \mathbb{K}_{r}$ ). That is, $w(t, x)$ is a generalized solution of the (CP) problem as in [11, Definition 1.1], except for replacing the term $f_{x} b(w)$ there by $(h f)_{x} \Sigma(w)$. The uniqueness of such non-negative $w \in C_{b}\left(\mathbb{R}_{T}\right)$, starting at the non-negative $w(0, x)=u(0, x) \in C_{b}(\mathbb{R})$, thus follows by adapting the proof of [11, Theorem $4.2(1)]$ to handle $h \not \equiv 1$.

To this end, we modify hereafter (7.18) as above and prove the analog of [11, (4.2)]. That is, we call $w$ a sub-solution if the r.h.s. of (7.18) is greater or equal its (modified) l.h.s. for every non-negative $f \in C^{1,2}\left(\mathbb{K}_{r}\right)$, any $r>0$, and all $t \in(0, T]$, while $w$ is a super-solution when the corresponding l.h.s. is greater or equal the r.h.s. for any such $f, r, t$. It then suffices to show that for some $c=c(h)$ finite, any super-solution $w$, all $t \in[0, T], \ell \in(0, \infty)$, and $[0,1]$-valued $\omega \in C_{c}^{\infty}([-\ell, \ell])$,

$$
\int_{\mathbb{R}}(u(t, x)-w(t, x)) \omega(x) \mathrm{d} x \leq c \int_{\mathbb{R}}(u(0, x)-w(0, x))_{+} \mathrm{d} x,
$$

where for any sub-solution $w$, the same inequality holds with $u-w$ replaced by $w-u$. Indeed, by definition of a super-solution (or sub-solution), the r.h.s. of (17.22) is zero. Hence, choosing $\omega(x)$ as smooth approximations of $\mathbf{1}_{u(t, x)>w(t, x)}$ on $(-\ell, \ell)$ and sending $\ell \rightarrow \infty$, we deduce that $m$-a.e. $u \leq w$ for super-solutions and $u \geq w$ for sub-solutions, yielding the stated uniqueness of the solution $w$. Fixing $t, \ell$, and $\omega$, we prove (7.22) for a given super-solution $w$ (exchanging the roles of $u$ and $w$ then yields the proof for sub-solutions). As in the proof of [11, (4.2)], for $f=f^{(n, r)}, n, r \geq \ell+1$ which solve suitable linear parabolic first boundary value problems, we bound the difference between the left most terms of (17.18) for $u$ and $w$. Taking $n \rightarrow \infty$ followed by $r \rightarrow \infty$ then yields (7.22).

Specifically, note that $\Sigma(u)-\Sigma(w)=(u-w) A_{\star}$ where

$$
A_{\star}=\int_{0}^{1} A(\tau u+(1-\tau) w) \mathrm{d} \tau
$$

is in $C_{b}\left(\mathbb{R}_{T}\right)$ and $A_{\star} \geq \underline{a}>0$. Hence, there exist uniformly bounded smooth functions $\left(A_{n}, B_{n}, C_{n}\right)$, such that $A_{n} \downarrow A_{\star}$ and $B_{n} \downarrow B_{\star}=-h A_{\star} \in C_{b}\left(\mathbb{R}_{T}\right)$, uniformly on $\mathbb{R}_{T}$, whereas $m$-a.e. $C_{n} \rightarrow C_{\star}=h_{x} A_{\star}$. Now, consider, for each $n$ and $r \geq \ell+1$, the unique classical solution $f^{(n, r)} \in C^{1,2}\left(\mathbb{K}_{r}^{o}\right)$ of the first boundary value problem

$$
\begin{array}{rlrl}
\mathcal{L}_{n} f:=f_{t}+A_{n} f_{x x}+B_{n} f_{x}-C_{n} f & =0 \quad \text { on } \quad(0, t) \times(-r, r), \\
f(t, x) & =\omega(x), & & x \in[-r, r], \\
f(s,-r)=f(s, r) & =0, & & s \in[0, t] .
\end{array}
$$

With $A_{n}$ bounded away from zero, the existence and uniqueness of such solution for (7.23) is well-known (see e.g. [29, chapter IV, Theorem 5.2]), and further, with $\omega \geq 0$ also $f^{(n, r)} \geq 0$. Next, setting $g_{n, r}^{ \pm}(s)=\left[f_{x}^{(n, r)}(\Sigma(u)-\Sigma(w))\right]\left(s, \pm r^{-}\right)$, we use the test function $f=f^{(n, r)}$ in our modified (7.18), to bound the l.h.s. of (7.22) by

$$
\begin{aligned}
& \int_{-r}^{r}(u(0, x)-w(0, x))_{+} f^{(n, r)}(0, x) \mathrm{d} x-\int_{0}^{t}\left[g_{n, r}^{+}(s)-g_{n, r}^{-}(s)\right] \mathrm{d} s \\
& +\int_{\mathbb{K}_{r}^{o}}(u-w)\left[f_{x x}^{(n, r)}\left(A_{\star}-A_{n}\right)+f_{x}^{(n, r)}\left(B_{\star}-B_{n}\right)-f^{(n, r)}\left(C_{\star}-C_{n}\right)\right] \mathrm{d} m .
\end{aligned}
$$


Recall the uniform boundedness of $u-w$ and the uniform convergence to zero of $A_{\star}-A_{n}$ and $B_{\star}-B_{n}$. Thus, similarly to the derivation of [11, (4.12)], the last term of (17.24) goes to zero when $n \rightarrow \infty$, provided $\sup _{s, x, n, r} f^{(n, r)}(s, x) \leq c$ finite and both $e_{n}:=\left\|f_{x x}^{(n, r)}\right\|_{L^{2}\left(\mathbb{K}_{r}\right)}$ and $\chi_{n}:=\left\|f_{x}^{(n, r)}\right\|_{\infty}$ are uniformly bounded in $n$. Taking then $r \rightarrow \infty$ yields (7.22), if in addition $\sup _{s, n}\left|g_{n, r}^{ \pm}(s)\right| \rightarrow 0$ as $r \rightarrow \infty$.

Turning to prove the latter four estimates, assume first that $m$-a.e. $h_{x} \geq 0$, hence $C_{n} \geq 0$ for all $n$ and $(s, x) \in \mathbb{R}_{T}$. Then, with $A_{n} \geq \underline{a}>0$, the maximum principle applies to the parabolic equation (7.23) (see e.g. [16, Sec. 2.1, Theorem 1], where our time direction is reversed compared to the setting there), resulting with $0 \leq$ $f^{(n)}(s, x) \leq \sup _{x} \omega(x) \leq 1$ for all $n, r$ (as in [11, Lemma 4.1, (i)]). Similarly, taking $\kappa>\sup _{n}\left\|A_{n}+\left|B_{n}\right|-C_{n}\right\|_{\infty}$ and $\mathbf{v}^{( \pm)}=f^{(n, r)}-\phi^{( \pm)}$for $\phi^{( \pm)}=e^{\ell \pm x+\kappa(t-s)}$, one has that $\mathcal{L}_{n} \mathbf{v}^{( \pm)} \geq 0$, while $\mathbf{v}^{( \pm)}(s, \pm r)=-\phi^{( \pm)}(s, \pm r) \leq 0$ and $\mathbf{v}^{( \pm)}(t, x)=\omega(x)-\phi^{( \pm)}(t, x) \leq 0$. Hence, by the maximum principle, both $\mathrm{v}^{(+)} \leq 0$ and $\mathrm{v}^{(-)} \leq 0$, yielding the bound $f^{(n, r)}(s, x) \leq e^{\ell-|x|}$ (as in [11, Lemma 4.1, (ii)]). Equipped with this bound, we follow the proof of [11, Lemma 4.1, (iii)]. Specifically, taking $\xi^{( \pm)}=y(r) e^{ \pm \kappa x}$ for $y(r)=$ $e^{\ell-(\kappa+1)(r-1)}$ and constant $\kappa \geq 1$ which makes $\kappa^{2} A_{n}-\kappa\left|B_{n}\right|-C_{n}$ non-negative on $\mathbb{R}_{T}$ for all $n$, results with $\mathcal{L}_{n} \mathbf{v}^{( \pm), \pm} \geq 0$ for $\mathbf{v}^{( \pm), \pm}=\xi^{( \pm)} \pm f$ and $f=f^{(n, r)}$. Thus, the four functions $\mathrm{v}^{( \pm), \pm}$satisfy the maximum principle on each of the two components of $\mathbb{K}_{r} \backslash \mathbb{K}_{r-1}$. Since $f(s, \pm r)=f(t, x)=0$ when $|x| \geq r-1$, while $|f(s, \pm(r-1))| \leq e^{\ell+1-r}$, by our choice of $\xi^{(+)}$, the maximum of $\mathbf{v}^{(+), \pm}$on the positive component of $\mathbb{K}_{r} \backslash \mathbb{K}_{r-1}$ is attained at $x=r$, where $\mathbf{v}^{(+), \pm}(s, r)=\xi^{(+)}(r)$ is constant. Hence, $\mathbf{v}_{x}^{(+), \pm}\left(s, r^{-}\right) \geq 0$ yielding that $\left|f_{x}\left(s, r^{-}\right)\right| \leq \kappa e^{\kappa} e^{\ell+1-r}$. Similarly, the maximum of $\mathrm{v}^{(-), \pm}$on the negative component of $\mathbb{K}_{r} \backslash \mathbb{K}_{r-1}$ is attained at $x=-r$, where $\mathbf{v}^{(-), \pm}(s,-r)=\xi^{(-)}(-r)$ is constant. Hence, $\mathbf{v}_{x}^{(-)}\left(s,-r^{-}\right) \leq 0$, so $\left|f_{x}\left(s,-r^{-}\right)\right| \leq \kappa e^{\kappa} e^{\ell+1-r}$, and $\sup _{s, n}\left|g_{n}^{ \pm}(s)\right| \rightarrow 0$ when $r \rightarrow \infty$. Having uniform ellipticity and $\left(A_{n}, B_{n}, C_{n}\right)$ uniformly bounded, the uniform bound on $\chi_{n}$ follows by applying [29, chapter III, Theorem 11.1] in our setting. Finally, to bound $e_{n}$ we multiply the linear PDE (17.23) by $f_{x x}$ and integrate over $\mathbb{K}_{r}$ as in the proof of [11, Lemma 4.1, (v)]. Following the derivation after [11, (4.10)], since $f_{t}^{(n, r)}(s, \pm r)=0$, integration by parts of the term $f_{t} f_{x x}$ results with

$$
\int_{\mathbb{K}_{r}^{o}} A_{n}\left(f_{x x}^{(n, r)}\right)^{2} \mathrm{~d} m \leq \frac{1}{2} \int_{-r}^{r} \omega^{\prime}(x)^{2} \mathrm{~d} x+\int_{\mathbb{K}_{r}^{o}} f_{x x}^{(n, r)}\left(C_{n} f^{(n, r)}-B_{n} f_{x}^{(n, r)}\right) \mathrm{d} m .
$$

Further, $\mathbb{K}_{r}$ is compact and $C_{n} f^{(n, r)}, B_{n} f_{x}^{(n, r)}$ uniformly bounded. Hence, by CauchySchwarz inequality, the rightmost term of (7.25) is bounded by $\kappa_{2} e_{n}$ for some $\kappa_{2}=$ $\kappa_{2}(r, h)$ finite. Further, the l.h.s. of (7.25) is at least $\underline{a} e_{n}^{2}$, whereas the first term on its r.h.s. is some $\kappa_{1}=\kappa_{1}(\omega)$ finite. Consequently, $\underline{a} e_{n}^{2} \leq \kappa_{1}+\kappa_{2} e_{n}$, yielding the desired uniform bound on $e_{n}$. This completes the proof in case $h_{x} \geq 0$. More generally, setting $c=e^{v T}$ for $v>\left\|\left(C_{\star}\right)_{-}\right\|_{\infty}$, the function $\widetilde{f}^{(n, r)}=e^{v(s-t)} f^{(n, r)}$ satisfies (7.23) with $\widetilde{C}_{n}=C_{n}+v \geq 0$. Thus, by the preceding $\sup f^{(n, r)} \leq c \sup \widetilde{f}^{(n, r)} \leq c, \sup _{n}\left\|g_{n, r}^{ \pm}\right\|_{\infty} \leq$ $c \sup _{n}\left\|\widetilde{g}_{n, r}^{ \pm}\right\|_{\infty} \rightarrow 0$ as $r \rightarrow \infty$, and $e_{n} \leq c\left\|\widetilde{f}_{x x}^{(n, r)}\right\|_{L^{2}\left(\mathbb{K}_{r}\right)}, \chi_{n} \leq c\left\|\widetilde{f}_{x}^{(n, r)}\right\|_{\infty}$ are both uniformly bounded in $n$, as claimed.

\section{REFERENCES}

[1] Aronson, D. G. (1968). Non-negative solutions of linear parabolic equations. Ann. Sc. Norm. Super. Pisa, Cl. Sci. Série 322 607-694.

[2] Banner, A. D., Fernholz, R., Karatzas, I. (2005). Atlas models of equity markets. Ann. Appl. Probab. 15 2296-2330. 
[3] Bass, R., Pardoux, E. (1987). Uniqueness for diffusions with piecewise constant coefficients. Probab. Theory Related Fields 76 557-572.

[4] Bossy, M., Talay, D. (1996). Convergence rate for the approximation of the limit law of weakly interacting particles: application to the Burgers equation. Ann. Appl. Probab. 6 818-861.

[5] Bossy, M., Talay, D. (1997). A stochastic particle method for the McKean-Vlasov and the Burgers equation. Mathematics of Computation 66 157-192.

[6] Chatterjee, S., Pal, S. (2010). A phase transition behavior for Brownian motions interacting through their ranks. Probab. Theory Related Fields 147 123-159.

[7] Chatterjee, S., Pal, S. (2011). A combinatorial analysis of interacting diffusions. J. Theor. Probab. 24 939-968.

[8] Dawson, D. A., Gärtner, J. (1987). Large deviations from the McKean-Vlasov limit for weakly interacting diffusions. Stochastics 20 247-308.

[9] Dembo, A., Zajic, T. (1995). Large deviations: from empirical mean and measure to partial sums processes. Stoch. Proc. Appl. 57 191-224.

[10] Dembo, A., Zeitouni, O. (1998). Large deviation techniques and applications. 2nd ed. Springer, New York.

[11] Diaz, J. I., Kersner, R. (1987). On a nonlinear degenerate parabolic equation in infiltration or evaporation through a porous medium. J. Differential Equations 69 368-403.

[12] Dudley, R. M. (2002). Real analysis and probability. 2nd ed. Cambridge University Press, New York.

[13] Dudley, R. M. (1999). Uniform central limit theorems. Cambridge University Press.

[14] Fernholz, E. R. (2002). Stochastic portfolio theory. Springer, New York.

[15] Fernholz, R., Karatzas, I. (2009). Stochastic portfolio theory: an Overview. In: Bensoussan, A., Zhang, Q. (eds.) Handbook of numerical analysis: Volume XV: Mathematical modeling and numerical methods in finance, 89-167. North Holland, Oxford.

[16] Friedman, A. (1964). Partial differential equations of parabolic type. Prentice-Hall, Inc. Englewood Cliffs, N. J.

[17] Gärtner, J. (1988). On the McKean-Vlasov limit for interacting diffusions. Math. Nachr. 137 197-248.

[18] Gilding, B. H. (1989). Improved theory for a nonlinear degenerate parabolic equation. Ann. Sc. Norm. Super. Pisa Cl. Sci. Série 416 165-224.

[19] Ichiba, T., Karatzas, I. (2009). On collisions of Brownian particles. Ann. Appl. Probab. 20 951977.

[20] Ichiba, T., Karatzas, I., Shkolnikov, M. (2011). Strong solutions of stochastic equations with rank-based coefficients. Probab. Theory Related Fields 156 229-248.

[21] Ichiba, T., Papathanakos, V., Banner, A., Karatzas, I., Fernholz, R. (2010). Hybrid Atlas models. Ann. Appl. Probab. 21 609-644.

[22] Jourdain, B. (2000). Diffusion processes associated with nonlinear evolution equations for signed measures. Methodology and computing in applied probability 2:1 69-91.

[23] Jourdain, B., Reygner, J. (2013) Propagation of chaos for rank-based interacting diffusions and long time behaviour of a scalar quasilinear parabolic equation. Stoc. Partial Diff. Eqns.: Anal. and Comp. 1 455-506.

[24] Karatzas, I., Shreve, S. (1998). Brownian motion and stochastic calculus. 2nd ed. Springer, New York.

[25] Krylov, N. V. (1982). Controlled diffusion processes. Springer, Berlin.

[26] Krylov, N. V. (1976). Sequences of convex functions and bounds on the maximum of the solution to parabolic equations. Siberian Math. J. 17 290-303.

[27] Krylov, N. V. (2007). Parabolic and elliptic equations with VMO coefficients. Comm. Partial Differential Equations 32 453-475.

[28] Krylov, N. V. (2012). Some $L_{p}$-estimates for elliptic and parabolic operators with measurable coefficients. Discrete and continuous dynamical systems Series B 17 2073-2090.

[29] Ladyzhenskaja, O. A., Solonnikov, V. A. and Ural'ceva, N. N. (1988). Linear and quasilinear equations of parabolic type. Translations of mathematical monographs 23. American Mathematical Society.

[30] Lieberman, G. (1996). Second order parabolic differential equations. World Scientific. River Edge, N.J. 
[31] McNamara, J. M. (1985). A regularity condition on the transition probability measure of a diffusion process. Stochastics 15 161-182.

[32] Oksendal, B. K. (2003). Stochastic differential equations. 6th ed. Springer, Berlin-Heidelberg.

[33] Pal, S., Pitman, J. (2008). One-dimensional Brownian particle systems with rank-dependent drifts. Ann. Appl. Probab. 18 2179-2207.

[34] Pal, S., Shkolnikov, M. (2014). Concentration of measure for systems of Brownian particles interacting through their ranks. Ann. Appl. Probab. 2414821508.

[35] Portenko, N. I. (1974). On the solutions of stochastic differential equations with integrable drift coefficient. Probability Theory and Its Applications XIX 577-582.

[36] Shkolnikov, M. (2010). Large systems of diffusions interacting through their ranks. Stoch. Proc. Appl. 122 1730-1747.

[37] Vázquez, J. L. (2007). The porous medium equation. Mathematical theory. Oxford mathematical monographs. The Clarendon Press, Oxford University Press, Oxford.

Departments of Statistics and Mathematics, Stanford University, Stanford, CA 94305

E-mail address: adembo@stat.stanford.edu

Department of Statistics, University of California Berkeley, CA 94720-3860

E-mail address: mshkolni@gmail.com

Courant Institute, New York University, New York, NY 10012-1185

E-mail address: varadhan@cims.nyu.edu

School of Mathematics, University of Minnesota, Minneapolis, MN 55455 and FaCulty of Mathematics, Weizmann Institute of Science, Rehovot, IsRael 76100

E-mail address: zeitouni@umn.edu 